University of Rhode Island

DigitalCommons@URI

Open Access Dissertations

1998

\title{
A Cluster Analytic Test of the Transtheoretical Model Applied to Exercise Behavior
}

Gregory J. Norman

University of Rhode Island

Follow this and additional works at: https://digitalcommons.uri.edu/oa_diss

\section{Recommended Citation}

Norman, Gregory J., "A Cluster Analytic Test of the Transtheoretical Model Applied to Exercise Behavior" (1998). Open Access Dissertations. Paper 951.

https://digitalcommons.uri.edu/oa_diss/951

This Dissertation is brought to you for free and open access by DigitalCommons@URI. It has been accepted for inclusion in Open Access Dissertations by an authorized administrator of DigitalCommons@URI. For more information, please contact digitalcommons-group@uri.edu. 


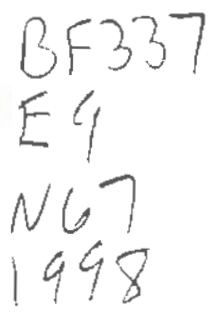

A CLUSTER ANALYTIC TEST OF THE

TRANSTHEORETICAL MODEL APPLIED TO EXERCISE BEHAVIOR BY

GREGORY J. NORMAN

A DISSERTATION SUBMITTED IN PARTIAL FULFILLMENT OF THE REQUTREMENTS FOR THE DEGREE OF DOCTOR OF PHILOSOPHY $\mathbb{N}$ PSYCHOLOGY

$$
41275234
$$

THE UNIVERSITY OF RHODE ISLAND 1998 


\section{ABSTRACT}

An empirical typology was created to test the application of the Transtheoretical Model (TTM) to exercise behavior. It was reasoned that if a meaningful typology of exercise could be created that depicted construct relationships posited by the TTM, then this would be supportive evidence for the application of the model to understanding behavior change related to exercise. A sample of 342 adults between the ages of 18 and 75 completed a survey through a random telephone interview. The sample was $62 \%$ female, $95 \%$ white, and an average age of 43 years. The sample was randomly split into two subsamples to perform cluster analysis on each subsample using Ward's method and squared Euclidean distance. The three variables used in the cluster analysis were the Pros

of exercise, the Cons of exercise, and Exercise Confidence. The pseudo F, pseudo $t^{2}$ and hierarchical dendrograms were used initially to determine the number of clusters and indicated either a five and six cluster solution. Comparison of cluster mean profile patterns for solutions 3 through 8 between the two subsamples resulted in choosing the 5 cluster solution as optimal. Four of the cluster patterns were found in both subsamples, while a fifth cluster was unique to each subsample. This resulted in 6 clusters that were labeled Disengaged, Early, At Risk, Early Action, Maintainers, and Habituated. A series of analyses were conducted to test the internal and external validity of the exercise typology. Two internal validity tests revealed that four of the cluster patterns demonstrated high stability and replicability, while the At Risk and Early Action cluster patterns were less stable. External validity of the clusters was demonstrated with significance testing using the six clusters as levels of a categorical variable to test for differences in five different variable domains: Exercise Behavior, Processes of Change, 
Stage of Change for Regular Exercise, Stage of Change for other health risks, and selected demographic variables. Large effect sizes were found for exercise behavior, processes of change, and stage of change. The clusters were also differentiated by stage of change for diet, gender, and perceived health. It was concluded that this series of analyses demonstrated the verisimilitude of the exercise cluster typology and that the clusters potentially represent a limited number of Stage of Change subtypes. The results also provide empirical evidence that the spectrum of behavior change can be represented as a series of discrete groups. Issues are discussed pertaining to the application of the exercise typology to developing interventions and recommendations for the use of cluster analysis for exploratory and confirmatory purposes. 


\section{ACKNOWLEDGMENT}

This dissertation is the culmination of several years of learning and collaborative research at the Cancer Prevention Research Center. I would like to thank my dissertation committee, which included Joseph Fava, Colleen Redding, Robert Sonstroem, and especially my major professor Wayne Velicer. His guidance over the last several years has been a strong influence on my thinking and development as a behavior scientist. I would also like to acknowledge James Prochaska, Joseph Rossi, and Lisa Harlow as professors who have had an important impact on me during my time as a graduate student. I must also thank Lynda Hurditch, Kathy Meier, and Elaine Taylor who I think of as my "other" committee.

My collaborators on exercise research, Joseph Rossi, Claudio Nigg, and Sonya Benisovich deserve a great deal of thanks for their work on the development of the questionnaire used for this project. I look forward to future collaborations with them. Thanks also to Julie Padula and the CPRC survey center for conducting the telephone interview assessments.

I feel privileged to have been a part of the $\mathrm{CPRC}$ at a time when talented individuals were emerging and developing their skills. Sara Johnson, Kerry Evers, Brett Plummer, and Jason Maddock are not only "hard-core" graduate students but valued friends. I would like to acknowledge other friends, both near and far, who have been an important part of my life over the last several years: Steve Pethick, Christina Ferguson, Todd Galati, Ann Hall, Tobey Demott, Jen Rose, and Chuck Persky. Life in Rhode Island would not have been the same, and not nearly as much fun, without the mountain 
bike madness crew of Larry Cronin, Bob Corr, Moe Chamberland, Colleen Kelly, Joe Clifford, Jim Case, and its newest member Sonya Benisovich. I would also like to acknowledge my parents and all of my family for their continued support and encouragement over the years. Finally, a special thanks to Sonya Benisovich who has brought incredible happiness to my life and has been very understanding and helpful during this project. 


\section{TABLE OF CONTENTS}

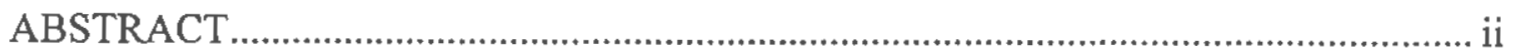

ACKNOWLEDGMENT .................................................................................... iv

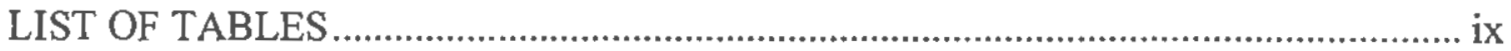

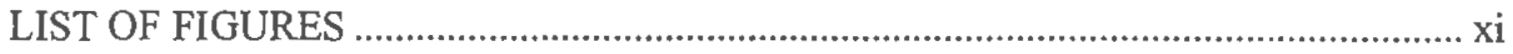

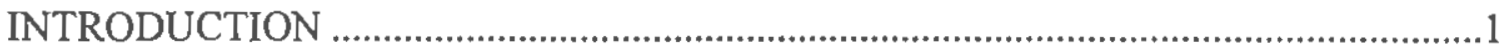

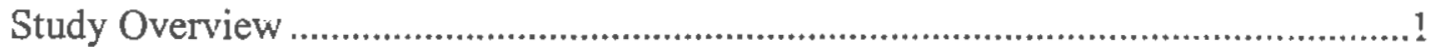

The Study of Exercise Behavior ....................................................................

Nature of the Problem ..................................................................................

Determining Qutcomes .........................................................................

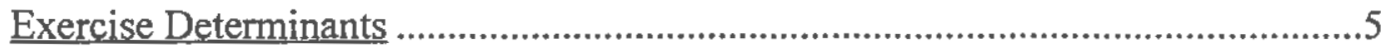

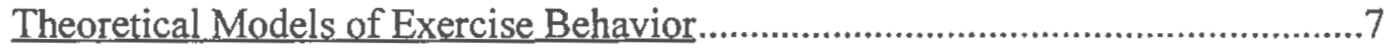

Transtheoretical Model and Exercise Behavior..................................................8

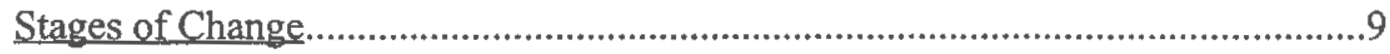

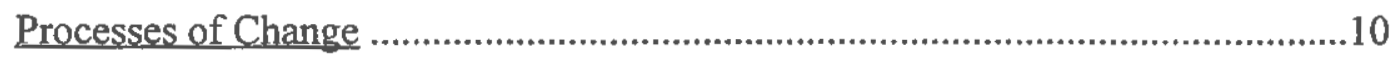

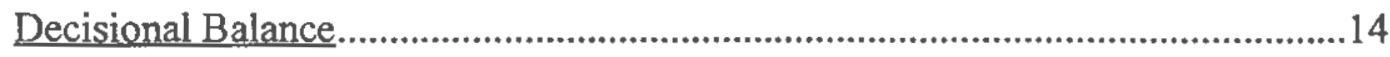

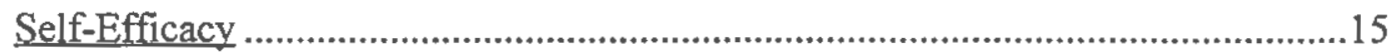

The Criterion Measurement Model and Stage Subtypes ..................................16

Cluster Analysis ......................................................................................18

An Alternative Method of Understanding Data ................................................. 18

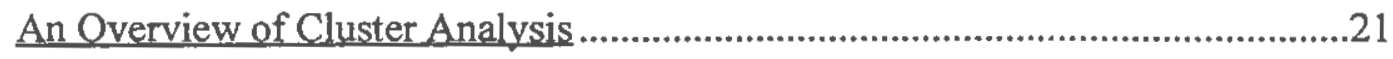

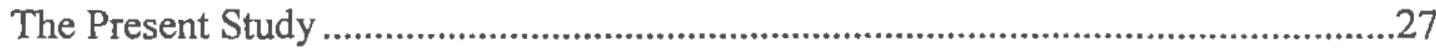


Hypotheses Regarding Cluster Validation.........................................................29

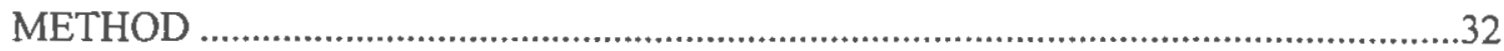

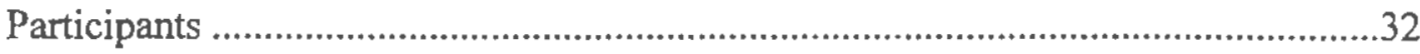

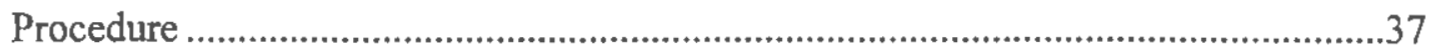

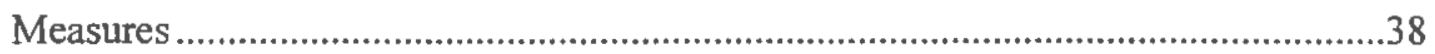

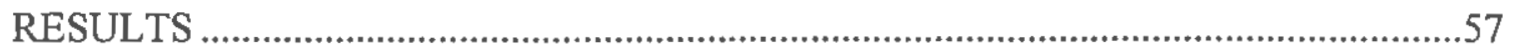

Preliminary Analysis .............................................................................................5

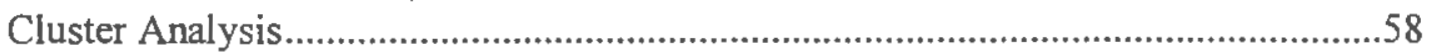

Describing and Naming the Clusters .....................................................................69

Intemal Validation: Random Subsample Clustering …..........................................73

Intemal Validation: Double Cross-Validation..........................................................76

Extemal Validation: Exercise and Physical Activity Behavior ....................................81

External Validation: Processes of Change ..................................................................8

External Validation: Stages of Change for Regular Exercise....................................94

External Validation: Stage of Change for Other Risk Factors.................................. 105

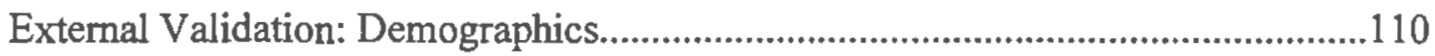

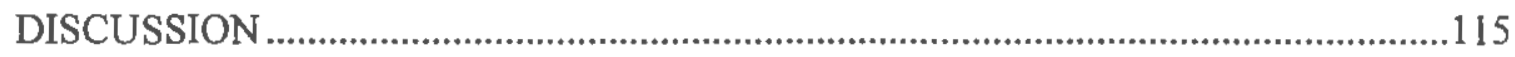

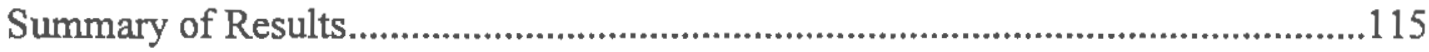

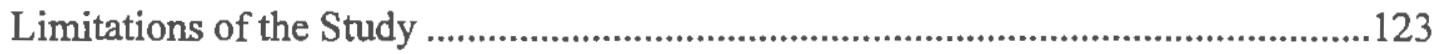

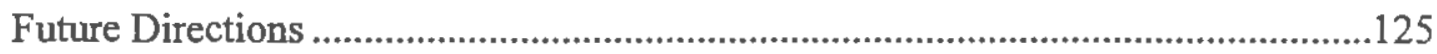

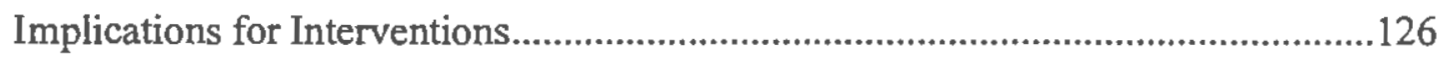


Conclusions.

APPENDIX

BIBILIOGRAPHY 


\section{LIST OF TABLES}

Table 1 Processes of Change for Exercise Behavior ................................................................................13

Table 2 Sample Characteristics: Demographics ........................................................................................34

Table 3 Sample Characteristics: Health Related.........................................................................................35

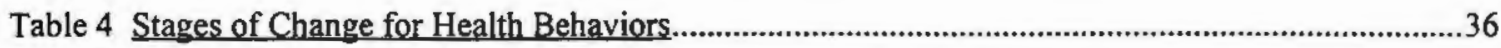

Table 5 Relationship Between Intension Based Staging and Marcus Based Staging ..................................47

Table 6 Items and Component Loadings for the Decisional Balance for Exercise Scale..............................48

Table 7 Raw Score Descriptive Statistics for Pros, Cons, and Confidence Summated Scales.....................49

Table 8 Items and Component Loadings for the Exercise Confidence Scale..............................................50

Table 9 Items and Factor Loadings for the 40 Item Exercise Processes of Change.....................................51

Table 10 Descriptive Statistics and Pearson Correlations for the Exercise Processes of Change ................54

Table 11 Means and Standard Deviations from the Paffenbarger/Harvard Alumni Questionnaire .............55

Table 12 Summary of Cluster Pattem Replication in Ten Random Subsamples .......................................75

Table 13 Cross-Validation of Subsample $2(n=165)$ Replicating Subsample $1(n=177) \ldots \ldots \ldots \ldots \ldots \ldots \ldots . . .79$

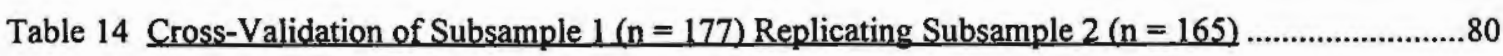

Table 15 Exercise Behavior Measures from Godin Leisure Time Exercise Questionnaire by Clusters ........84

Table 16 Experiential Processes of Change by Clusters..............................................................................90

Table 17 Behavioral Processes of Change by Clusters.................................................................................91

Table 18 Two-Way Table for Exercise Clusters and Stage of Change for Exercise ...................................98

Table 19 Relationship Between Clusters and Stages of Change ..............................................................99

Table 20 Two-Way Table for Exercise Clusters and Stage of Change for Diet.........................................107

Table 21 Two-Way Table for Exercise Clusters and Stage of Change for Sun Protection .........................108

Table 22 Two-Way Table for Exercise Clusters and Stage of Change for Smoking ................................109

Table 23 Two-Way Table for Exercise Clusters and Gender................................................................112

Table 24 Two-Way Table for Exercise Clusters and Perceived Health ................................................. 113

Table 25 Descriptive Statistics and ANOVA Results for Demographic Variables by Exercise Clusters ..114 
Table 26 Summary of Cluster Analysis and Validating Procedures .. 


\section{LIST OF FIGURES}

Figure 1. Flow chart depicting the stages of change algorithm. .56

Figure 2. Dendrograms from the cluster analysis of the two data subsamples. .62

Figure 3. Profile patterns for the 3 cluster solution. .63

Figure 4. Profile pattems for the 4 cluster solution. 64

Figure 5. Profile patterns for the 5 cluster solution. 65

Figure 6. Profile patterns for the 6 cluster solution. 66

Eigure 7. Profile pattems for the 7 cluster solution. 67

Figure 8. Profile pattems for the 8 chuster solution....... 68

Figure 9. Six cluster profiles based on Pros, Cons, and Confidence. .72

Figure 10. Self-reported level of leisure time exercise (METS) by cluster. 85

Figure 11. Weekly self-reported estimates of stenuous, moderate, and mild exercise by cluster. 86

Figure 12. Experiential process use by clusters. .92

Figure 13. Behavioral process use by clusters. .93

Figure 14. Individual profiles by cluster for the Precontemplation stage. 100

Figure 15. Individual profiles by cluster for the Contemplation stage. 101

Figure 16. Individual profiles by cluster for the Preparation stage. 102

Figure 17. Individual profiles by cluster for the Action stage. 103

Eigure 18. Individual profiles by cluster for the Maintenance stage. 104

Eigure 19. Three level conceptualization of the TTM based intervention approaches. 133 


\section{A CLUSTER ANALYTIC TEST OF THE \\ TRANSTHEORETICAL MODEL APPLIED TO EXERCISE BEHAVIOR}

Introduction

Study Overview

The purpose of the present study was to create an empirical typology of exercisers based on constructs from the Transtheoretical model (TTM) of behavior change. The study involved clustering individuals based on the multivariate outcome space defined by the TTM (Pros of exercise, Cons of exercise, and Confidence to exercise) to determine if homogeneous groups existed. Several possible outcomes were hypothesized for an empirical typology of exercise behavior. These outcomes included: (a) no discernible clusters, (b) a small number of clusters corresponding to the Stages of Change, (c) a small number of clusters that do not support the TTM but have some kind of meaningful interpretation, (d) a larger number of clusters corresponding to the stage subtypes previously found for smoking cessation, and (e) a larger typology of clusters that does not support the TTM. Each of the five outcome possibilities was interpretable in the context of testing the TTM. Outcomes (b) and (d) were considered supporting evidence for the model's application to exercise behavior, while the other three outcomes were not considered supportive evidence.

The resulting exercise cluster typology was validated in a series of analyses. Internal validity was assessed by testing the replicability of the cluster patterns by clustering random subsamples from the data set as well as through a double cross- 
validation procedure. External validity was examined by using significance testing with the exercise clusters as the levels of the independent variable and several groups of relevant variables. First, differences in the clusters were tested on behavior with two selfreport activity measures. Second, the ten processes of change served as dependent measures in multivariate analysis of variance (MANOVA). Third, the relationship between stage of change for regular exercise and the clusters was examined. Fourth, the association between the cluster typology and stage of change for three health behavior risk factors (smoking, diet, sun protection) was assessed to determine if more physically active clusters were also further along in the stages for other important health behaviors. Finally, differences in the clusters were tested for several demographic variables such as gender, age, education level, and body mass index.

The goal of the present study was to address several broad research questions:

1. Can a meaningful empirical typology of exercise behavior change be created based on three cognitive constructs?

2. What is the verisimilitude of the cluster groups? This question addresses both the nature of the resulting typology and the utility of the cluster analysis procedure.

3. Does the empirical exercise typology support construct relationships posited by the TTM? This question addresses the confirmatory purposes of the study.

4. What new information can be learned about the application of the TTM to exercise behavior by using cluster analysis? This question pertains to the exploratory aspect of the study. 
An additional purpose of the present study was to provide an in depth presentation on the use of cluster analysis and cluster validating procedures that can serve as guidance to others interested in using the procedure.

The remaining sections of the introduction review some of the previous research on exercise behavior, provide a review of the TTM and its application to exercise behavior, explain cluster analysis methodology, and further describe the present study.

\section{The Study of Exercise Behavior}

\section{Nature of the Problem}

The benefits of regular exercise have been well established. Reed (1995) has compiled a list of 50 benefits relating to physical and mental well-being, reduction of the risk of chronic diseases, and the enhancement of rehabilitation. What is not well understood is how to get people to become more physically active and how to keep them active. In the US population the statistics relating to exercise behavior are alarming. It is estimated that $12 \%$ of all deaths per year in the US are related to lack of regular exercise (Pate et al., 1995). Blair et al. (1993) estimated that 20,000 fewer deaths per year would occur if half of those who participate in no leisure-time physical activity would do something just a few times per week. Less than $10 \%$ of people exercise vigorously at levels necessary for achieving health benefits (Sallis \& Hovell, 1990). The problem is not confined to the US. It has been estimated that 60 percent of all adults in modern western societies are not exercising at recommended levels for achieving health benefits (Buxton, Wyse \& Mercer, 1996). In terms of moderate physical activity (3-6 METS), 
only about $22 \%$ exercise at adequate levels of physical activity, $54 \%$ are somewhat active, and $24 \%$ of the population are completely sedentary (Pate et al., 1995). Finally, Dishman (1988) has noted that $50 \%$ of individuals who join an exercise program will drop out within the first three to six months. Thus, behavior scientists are faced with two very important questions: (a) How to get people to adopt physical activity as part of their lifestyle, and (b) How to help physically active people maintain their exercise habits.

\section{Determining Outcomes}

Exercise adoption and maintenance have been difficult to understand and investigate because the outcome criteria for regular exercise behavior has varied and has changed over time (Pate et al., 1995; King et al., 1992). Without clear objective definitions of what constitutes regular exercise behavior it is difficult to interpret results across studies. The original Amercian College of Sports Medicine (ACSM) (1990) guidelines for exercise were that health benefits resulted from vigorous exercise (20 to 60 minutes at 60 to $90 \%$ of maximum capacity) at least three days a week. More recent research has indicated that nearly all of the health benefits of exercise can be obtained from moderate physical activity. The new ACSM guidelines recommend activity at an intensity of 3 to 6 METS (e.g. brisk walking) which can be accrued in bouts as short as ten minutes totaling about 30 minutes of activity each day. It is important to point out that there is a dose response with greater benefits acquired from more intense exercise, and that these new guidelines serve as a supplement to the vigorous exercise recommendations and do not succeed them. 
Exercise Determinants

Much of the research on exercise behavior has focused on various determinants of exercise. Although some of the more recent studies were prospective, Dishman accurately notes that since much of this research on exercise determinants has been crosssectional, correlational studies, determinants should really be defined as reproducible associations rather than cause and effect relationships (Dishman, 1990; Dishman \& Sallis, 1993). Several comprehensive and critical reviews of the exercise determinants literature have been published (Sallis \& Hovell, 1990; Dishman, 1990; King et al., 1992; Dishman \& Sallis, 1993). This section summarizes the different types of determinants that have been studied and their relationship to exercise behavior. Three broad categories of determinants have been identified: personal attributes, environmental factors, and physical activity itself.

Personal Attributes. Personal attributes includes several domains of determinants related to the individual such as demographics, past and present behavior, psychological beliefs, attitudes, as well as specific knowledge and behavioral skills. Demographic characteristics associated with sedentary behavior include being elderly, black, female, and poor (King et al., 1992). However, age is more likely to be a selection bias rather than a cause of inactivity (Dishman, 1990). The overweight are less likely to remain in fitness programs. Occupation and smoking status have not shown a relationship with exercise behavior. Past program participation is the most reliable predictor of current participation in supervised exercise programs. Perceived health, self-efficacy, self- 
motivation, and perceived exercise enjoyment and satisfaction have been found to be consistent correlates with physical activity. However, King et al. (1992) point out that intention to exercise has consistently shown to be a poor predictor of exercise behavior. Indicating that intention may be necessary but not sufficient relating to exercise behavior. Dishman (1990) reports that active peopie appear to be more knowledgeable about the benefits of exercise but it is unclear whether such knowledge is an antecedent or consequence of activity. Finally, behavioral skills such as effective goal setting, selfmonitoring of progress, and self-reinforcement have been bound to be positively correlated with maintaining physical activity.

Environmental Factors. Determinants which are external factors in and about the individual's environment can be divided into social supports, time, access to facilities, and climate conditions. Family participation and support has been shown to be strong predictors of exercise maintenance for women but not for men (King et al., 1992). There is some cross-sectional evidence of peer support as a predictor of vigorous exercise in young men and women (King et al., 1992). The most common reported excuse for not exercising is perceived lack of time. However, this is also the most common concern of regular exercisers, indicating that for sedentary individuals lack of time may reflect lack of interest or commitment to physical activity (Dishman, 1990). A consistent predictor of exercise participation has been access to exercise facilities in terms of the distance from an individual's home or place of employment. Weather conditions have been shown to be a consistent barrier to physical activity to all but the most committed of exercisers. 
Seasonal availability and climate conditions are notable influences on choice of out-door activities (Dishman, 1990).

Physical Activity Characteristics. There is no research evidence to indicate that activity intensity, perceived discomfort, or frequency influence drop out rates or for supervised exercise programs. Little research has been conducted on the effects of monetary costs and program formats to draw any conclusions about these factors.

\section{Theoretical Models of Exercise Behavior}

The study of exercise behavior has been criticized for being largely atheoretical (Dishman, 1988; Sonstroem, 1988; Sallis \& Hovell, 1990; Godin \& Shepard, 1990). Focusing on the determinants of exercise behavior has led to static models which isolate specific predictors rather than attempting to meaningfully integrate relevant constructs. Determinants such as gender, education, climate, and family makeup, have limited value because they can not be impacted by interventions. Sonstroem (1988) and others have called for the use of more dynamic models which recognize that exercise behavior is a process that involves several steps with varying determinants at each step. Sallis and Hovell (1990) proposed that exercise behavior can be conceptualized as a series of phases and transitions. These phases were labeled sedentary, adoption, maintenance, dropout, and resumption. The important transitions are: from sedentary to adoption, where there has been very little research; from adoption to maintenance or dropout, which has received the most attention; and from dropout to resumption where almost no research has been conducted. Identifying these phases and transitions not only points out where 
research needs to be focused, but it indicates the need for implementing more multidimensional models of exercise behavior.

Some exercise researchers have begun to apply, or at least conceptualize, exercise within more multivariate models such as the Theory of Reasoned Action (Godin, 1994), Social Cognitive Theory (Dzewltowski, 1994), and the Transtheoretical Model (Marcus \& Simkin, 1994; Prochaska \& Marcus, 1995). These models share more similarities than they do differences, since they all have roots in earlier outcome-expectancy values theories (Dishman, 1994; King et al. 1992). Each theory has a decision-making or costbenefits analysis component, and each emphasizes the importance of self-efficacy as a predictor of behavioral outcomes. The Transtheoretical Model (TTM) is the most integrative of these theories. Cognitive constructs along with a set of behavior change strategies are integrated along a temporal dimension called the stages of change. The model reflects the natural phases and transitions of exercise discussed by Sallis and Hovell (1990) and includes many of the more effective determinants of exercise. The TTM also facilitates the development of exercise interventions by matching specific strategies to individuals based on their readiness to change.

\section{Transtheoretical Model and Exercise Behavior}

In this section the constructs within the Transtheoretical model are described along with how the model has been applied to exercise behavior. 


\section{Stages of Change}

The stages of change are the temporal dimension in which change unfolds. Stages can be conceptualized as falling in between states and traits, having both stable and dynamic qualities. Stages are stable over time yet open to change (Prochaska \& Marcus, 1995). The length of time an individual spends in a stage may vary but the activities a person must complete to successfully move to the next stage are invariant. Each stage is defined by intentions and behaviors related to the problem behavior of interest.

Five stages are included in the current TTM. Precontemplation (PC) is characterized by resistance to recognizing and modifying a problem behavior. The Problem for PCs is not that they do not see the solution but that they do not see the problem. In exercise PCs have no intention of beginning to exercise in the next six months. Contemplators $(\mathrm{C})$ are individuals who are seriously considering exercising in the next six months. They recognize the problem, they know what they want to do, they are just not ready to act yet. The Preparation (PR) stage is characterized by both intentions to take action in the near future (next 30 days) and small behavioral changes. People in PR for regular exercise have started to exercise some but not regularly but intend to do so soon. The Action (A) stage is where behavior is being performed at the criterion level. The individual is either exercising at the recommended moderate or vigorous levels but has been doing so for less than six months. The Maintenance (M) stage is not a static stage but where the individual works to consolidate gains made working through the stages and to avoid relapse. Because exercise involves adopting and 
maintaining a new behavior, maintenance may be considered a life long stage for exercise.

The stages of change are an important concept for exercise because they accurately portray the revolving door nature of exercise behavior (Reed, Velicer, Prochaska, Rossi, \& Marcus 1997). An assumption of the stage construct is that individuals do not progress once through the stages but may cycle through many times beginning, maintaining, and relapsing, and resuming exercise programs.

Stage of change for regular exercise has been measured with a number of different types of assessments (Reed et al., 1997; Booth et al. 1993; Marcus \& Simkin, 1993). These include 5-point ordered categorical measures, multiple question Yes/No algorithms, and multiple question inventories that generate a scale score for all five stages for each individual (Reed, 1995; Reed et al.. 1997). The first two categorical methods of staging are the most commonly employed and have demonstrated adequate test-retest reliability and concurrent validity in a number of studies (Cardinal, 1997; Cardinal, 1995; Buxton, Hale, Mercer, Wyse, \& Ashford, 1994).

\section{Processes of Change}

While the stages of change reflect when change occurs, the processes of change are the strategies individuals use to make changes. The processes were originally formulated from examining methods of therapy from the major psychological theories (Prochaska, 1979). Processes represent an intermediate level of abstraction between these theoretical positions and the diverse specific therapy strategies derived from the theories 
(Prochaska, DiClemente, \& Norcross, 1992). Thus, although there are hundreds of strategies for changing behavior, they can be classified within ten common processes of change. It was also found that these ten processes described the strategies that selfchange smokers were using on their own (Prochaska \& DiClemente, 1983). Ten scales were developed to measure the processes assessing the frequency of their use for smoking cessation (Prochaska, Velicer, DiClemente, \& Fava, 1988). The measurement model revealed that the latent structure of the ten processes can be modeled as two correlated higher order factors, labeled experiential and behavioral, each with five first order processes factors. Examining the relationship between processes of change and the stages of change revealed that process use varies systematically across the stages. Experiential process use tends to peak in the Contemplation and Preparation stages, while Behavioral process use tends to peak between the Action and Maintenance stages. This pattern has been shown cross-sectionally (Prochaska \& DiClemente, 1983) and prospectively (Prochaska, Velicer, DiClemente, Rossi, \& Guadagnoli, 1991).

Measurement development of the processes of change for exercise was conducted by Marcus, Rossi, Selby, Niaura, \& Abrams (1992) by adapting the processes of change for smoking measures (Prochaska et al., 1988). Many of the processes involved the determinants of exercise that were previously outlined. In cross-sectional analysis, Marcus, Rossi et al. (1992) demonstrated that the structure of the ten processes of change fit well for exercise. One important difference between exercise and smoking was that while behavioral processes tended to peak in the action stage for smoking they did not differentiate exercisers in the action and maintenance stages. This difference may result 
from exercise being an acquisition behavior rather than a cessation behavior. Table 1 provides a definition and example of each of the ten processes of change adapted for exercise behavior. 
Table 1

Processes of Change for Exercise Behavior

Processes of Change Description

Experiential

Consciousness Raising Gaining information and knowledge about the health benefits of exercise and the health risks of a sedentary lifestyle.

Dramatic Relief

Self Reevaluation

Environmental

Reevaluation

Social Liberation

Behavioral

Self Liberation

Stimulus Control

Counter Conditioning

Reinforcement

Management

Helping Relationships
Emotional reactions from learning about the health risks of not exercising.

An assessment of how exercising regularly relates to personal self-standards and values.

Consideration of how being a regular exerciser may influence other people such as friends, family, and coworkers.

Consideration of how the environment is changing to facilitate exercise behavior. Examples include, noticing exercise programs at work or walking trails near the home.

Making commitments to regular exercise and taking personal responsibility for one's own exercise behavior.

Restructuring the environment to make exercising easier. Examples include, putting the gym bag by the front door, avoiding people who encourage sedentary behavior.

The substitution of healthy behaviors for unhealthy behavior. Examples include, taking a walk instead of staying on the couch, or exercising instead of making excuses for skipping exercise.

Providing positive rewards and incentives for exercising regularly.

Having important others for support. Examples include having an exercise partner or simply someone to talk to when motivation to exercise is low. 


\section{Decisional Balance}

Decisional balance is a measure of the importance of reasons and concerns relating to making a change in behavior. Velicer, DiClemente, Prochaska, \& Brandenburg (1985) developed this measure for smoking cessation based on Janis and Mann's (1977) model of decision theory. The original model hypothesized four positive and negative aspects to consider when making a decision. These included, utilitarian gains and losses to self, gains and losses to others, and self-approval and self-disapproval, and approval and disapproval from important others. Velicer et al. (1985) found that the structure of the decision to change smoking behavior consisted of two constructs, the Pros (positive aspects) and the Cons (negative aspects) of change.

Progressing through the stages of change can be described in terms of the Pros and Cons. In smoking, PC is characterized by high importance of the pros of smoking and low importance of the cons. In $\mathrm{C}$ the Cons have increased in importance creating conflict for the individual between the perceived benefits and drawbacks of smoking. In PR the Cons become more important than the Pros which is represented by a crossover effect of the two variables. In $\mathrm{A}$ and $\mathrm{M}$ the Cons of smoking remain higher than the Pros but both decline over time indicating that once the decision to quit has been made other variables become more important. Also if action is taken before the Cons out weigh the Pros an individual is at high risk for relapse because they were not quite cognitively ready to take action. The characteristic crossover effect occurring in either Preparation or Contemplation has been identified in the process of changing twelve different health related behaviors (Prochaska, Velicer et al., 1994). Prochaska (1994) has also 
hypothesized the magnitude of change that has to occur in the Pros and Cons scales for a person to make a behavior change.

Marcus, Rakowski, and Rossi (1992) developed a decisional balance inventory for exercise. Pros items reflect the importance of how exercise makes one feel, improving confidence, providing energy, and how exercise can help one to sleep more soundly. The Cons items reflect the importance of perceived barriers and drawbacks to exercise such as being too tired, not having enough time for family, and that exercise is uncomfortable. A pattern similar to that of smoking has been found for decisional balance for exercise across the stages of change in US and Australian adult worksite samples (Marcus, Rakowski, \& Rossi, 1992; Marcus \& Owen, 1992; Marcus, Pinto, Simkin, Audrain, \& Taylor, 1994), older Australian adults (Gorely \& Gordon, 1995), and Canadian high school students (Nigg \& Courneya, 1997).

\section{Self-Efficacy}

Bandura $(1977,1982)$ conceptualized self-efficacy as a person's perceived ability or confidence to perform a given task. These efficacy expectations are a mediator of future performance and as a result, changes in self-efficacy are predictive of changes in behavior. An alternative perspective on this concept is from models of relapse prevention (Brownell, Marlatt, Lichtenstein, \& Wilson, 1986) that have focused on typologies of situations in which relapse is likely to occur. Investigating smoking behavior, Velicer, DiClemente, Rossi, and Prochaska (1990) integrated these two perspectives into a three factor hierarchical measurement model. The three scales were labeled Positive/Social, 
Negative/Affective, and Habit/Addictive. These three constructs were found to be related to a higher-order general factor. Inventories assessing both self-efficacy and temptations fit this same three factor model. Both constructs have systematic relationships across the stages of change. Self-efficacy increases linearly from PC to M, while temptations decrease from $\mathrm{PC}$ to $\mathrm{M}$.

In exercise behavior, self-efficacy has been conceptualized as one's confidence to exercise under less than desirable conditions (Sallis, Pinski, Grossman, Patterson \& Nader, 1988). Confidence in one's ability to exercise has been considered an important determinant of exercise behavior. A five item scale measuring confidence to exercise during bad moods, bad weather, while on vacation, when there is little time, and when tired was developed by Marcus, Selby, Niaura, \& Rossi (1992) and demonstrated the ability to differentiate the stages of change. Precontemplators reported the lowest confidence and those in Maintenance reported the highest confidence to exercise regularly. Recently, a hierarchical multidimensional exercise self-efficacy measure was developed which identified six components of self-efficacy labeled Negative Affect, Excuse Making, Exercising Alone, Access to Equipment, Resistance from Others, and Weather (Benisovich, Rossi, Norman, \& Nigg, 1998). A similar linear increasing pattern was also found for these scales across the stages of change.

\section{The Criterion Measurement Model and Stage Subtypes}

The constructs of Decisional Balance and Self-Efficacay have been incorporated by Velicer, Rossi, Prochaska, and DiClemente (1996) into a recently proposed criterion 
measurement model (CMM). The CMM was developed within the context of smoking behavior as an alternative to the traditional problematic point prevalence outcome variable. The model was proposed as a means to measure the full spectrum of the change process as an individual progresses from being a smoker to becoming a non smoker. The advantages of the CMM over traditional outcome variables are that the model consists of constructs rather than simply variables, it is multivariate rather than univariate, its measures are continuous rather than discrete, and it can measure longitudinal change rather than only cross-sectional change. In the CMM the three constructs were identified as Habit Strength, the physiological and psychological aspects of smoking behavior; Positive Evaluation Strength, the importance of positive values and beliefs about smoking; and Negative Evaluation Strength, the importance of negative values and beliefs about smoking. In the present study, these three constructs were measured with scales assessing exercise confidence, the pros of exercise, and the cons of exercise, to form the basis for creating the empirical exercise typology.

The constructs from the CMM have been used as the basis for the analysis of subtype groups within the stages of change. The idea of stage subtypes was first suggested by Heather (1991) and was empirically investigated by Velicer, Hughes, Fava and Prochaska (1995) using an accidental sample of smokers and former smokers, and by Norman, Velicer, Fava, and Prochaska (1997) in a representative sample of smokers. Subtypes may be useful as an intermediate level of treatment-matching between broad group interventions and completely individualized treatment strategies. 
In the Velicer et al. (1995) study, cluster analysis within each of the first four stages of change (Precontemplation, Contemplation, Preparation, Action) revealed distinct subtypes that shared similar characteristics across the four study groups. In each stage, a profile was found which exemplified that stage, labeled Classic; a profile that was similar to the next stage, labeled Progressing; and a profile that was similar to the previous stage (except for Precontemplation), labeled Early. Within each stage an unexpected profile was also found that was labeled Disengaged because these cluster profiles reflected detachment or disinterest in the cognitive and physiological aspects related to smoking. The cluster subtypes were interpreted as strong support for conceptualizing the five Stages of Change as discrete stages. The Progressing and Early subtypes represented potential stage algorithm misclassifications indicating that some individuals perhaps belong in the preceding or succeeding stage. In the Norman et al. (1997) study a typology of subtypes was found that closely resembled the findings from the accidental sample. The main difference was that in the representative sample, an additional Disengaged subtype was found in the Precontemplation and Preparation stages of change.

\section{Cluster Analysis}

\section{An Alternative Method of Understanding Data}

The field of psychology has struggled throughout its history with a distinction between two seemingly contrasting focuses. On the one hand, there is the psychology that is concerned with the development of general laws or common patterns of behavior. 
On the other hand, there is the psychology which emphasizes the uniqueness of the individual. Allport (1937) borrowed the terms nomothetic and idiographic from the German philosopher Windelband to describe these two psychological approaches. This contrast of focuses creates a dilemma for psychologists which is also reflected in Henry Murray's statement, "Every man is in certain respects like all other men, like some other men, and like no other men." (Kluckhohn, Murray, \& Schneider, 1953, p. 53).

The tension between the nomothetic and idiographic focuses goes beyond its origins in personality psychology and extends to the very way in which research is conducted. This point was the topic of Cronbach's 1957 presidential address to the American Psychological Association. Cronbach (1957) described psychology as two disciplines. Experimental psychology had a nomothetic focus and conducted research experiments to discover between group variation. Typically in this kind of research, the emphasis was on central tendency and linear relationships between variables and within group variation was labeled noise or error and ideally should be controlled and minimized. Correlational psychology took the opposite approach of experimental psychology and focused on individual differences. Here the approach was descriptive and variables that reflected differential variation to external conditions were of primary interest.

Continuing from Cronbach, many other psychologists have written about the nomothetic and idiographic poles of psychology. The consensus seems to be to find ways that one approach can contribute to the other to facilitate research toward understanding behavior (Howard \& Myers, 1990; Dunn, 1994; Franck, 1982). Through efforts to 
synthesize the nomothetic/idiographic and the experimental/correlational approaches to psychology, there has been an increased understanding that research methods and observations are inherently biased and presumptive. The type of analysis that is used shapes the evidence that is generated and what can be known (Phillips, 1987; Waller \& Meehl, 1998). A convergence of evidence from a variety of perspectives (types of analyses) can generate confidence and support for knowledge claims.

Cluster analysis is a multivariate procedure that offers an alternative method of understanding data. Cluster analysis can be seen as falling in-between traditional linear analysis of central tendency and ad hoc correlational analysis. Rapkin and Luke (1993) discussed how cluster analysis can be used in place of linear model analyses which may misrepresent complex multifaceted relationships as random error variance. When a sample contains a mixture of cases with different combinations of relationships among key variables, linear model analyses may obscure these relationships and treat them as noise. Different types may exist in the sample representing different multivariate profiles. There may be a group that conforms to the aggregate's central tendency on the key variables with the addition of several other groups that represent other patterns. For these groups their values represent 'error' with respect to the central tendency of the sample but their pattern of variable relationships is also of importance to understanding the whole sample.

The dendrogram tree from hierarchical agglomerative cluster analysis can be used to represent the continuum from nomothetic to idiographic. This tree begins at the roots (branches) with all individuals represented as separate. Here there is at maximum 
complexity, each individual in the sample is their own group. No meaningful statements can be made about the sample, as everyone is a unique case. At the other end of the tree, all individuals have been grouped into one cluster. Simplicity is maximized but meaningful statements still can not be made about the sample, all individuals are part of one group. The goal of the researcher using cluster analysis is to find the level of the dendrogram tree that meaningfully represents the individual profiles as a limited number of cluster groups.

A goal of the present study was to show how cluster analysis can be combined with other statistical procedures to integrate the nomothetic and idiographic approaches to exploring and analyzing data in meaningful ways by taking into account typical patterns of change and individual patterns of change.

\section{An Overview of Cluster Analysis}

This section provides a brief overview of cluster analysis. Detailed and comprehensive explanations of cluster analysis are available in monographs by Everitt (1980), Lorr (1983) and Aldenderfer and Blashfield (1984).

Cluster analysis is a multivariate classification method that uses a series of steps to identify homogeneous subgroups within a data set. This is a type of parsimony procedure where the goal is to reduce $\underline{N}$ subjects down to $\underline{M}$ groups $(\underline{N}>\underline{M})$ based on $p$ variables. The selection of variables to be used in cluster analysis is a critical step. This decision should be based primarily on theoretical considerations. The researcher should ask the question, "What variables would best characterize possible groups within the 
data?" Cluster analysis performs optimally when relevant variables are included and irrelevant variables are excluded from the analysis. There is no set limit to the number of variables one can use in a cluster analysis (except for the limits of your computer). However, results are more easily interpreted when a limited number of variables are selected to cluster individuals. It is much easier to interpret and display clusters in four or five dimensional space than in ten dimensional space. Variables used in cluster analysis should also be relatively independent of each other. This makes intuitive sense since highly correlated variables do not add any significant information to the cluster analysis. Thus, when selecting variables it is desirable to choose a small number of uncorrelated relevant variables that will best characterize potential groups within the data.

The variables selected for a cluster analysis are generally standardized to a comparable scale. Standardizing the variables equalizes the contribution of each variable in order to avoid unintentional variable weighting. Standardization of variables is particularly important when the variables have substantially different units of measurement (Aldenderfer \& Blashfield, 1984). However, there is some controversy with regard to whether or not to standardize variables in cluster analysis. Aldenderfer and Blashfield (1984) and Gordon (1980) discuss some of the advantages and disadvantages when making this decision. Simulation studies on the performance of various cluster algorithms conducted by Edelbrock (1979) and Milligan (1980) demonstrated that the standardization of variables had only a minor effect on cluster solutions.

Once variables have been selected and standardized, a measure of how each individual relates to every other individual in $\boldsymbol{p}$ dimensional space must be 
determined. This is accomplished by creating an $\underline{N} \times \underline{N}$ matrix of either similarity or distance coefficients. To determine which of these types of coefficients to use, it is necessary to understand how they represent the $\underline{N}$ objects in $p$ dimensional space. If the rows of the $\underline{N} \times p$ data matrix are thought of as representing individual profiles, then these profiles can be decomposed into three descriptive properties which define the profile and its relationship to the other $\underline{N}-1$ individual profiles. These properties are called elevation, scatter, and shape. The elevation of the profile, also called level or height, is the mean score of the $p$ variables for an individual. The scatter is the dispersion of the profile's scores around their average, or the standard deviation within the profile. The shape represents the configuration of the scores, or the dips and rises across the variables (Aldenderfer \& Blashfield, 1984; Cronbach \& Gleser, 1953).

We can now consider how similarity and distance coefficients relate to these profile properties. The Pearson $\underline{R}$ is a type of similarity measure, but in this case it is bounded by 0 and 1 with higher numbers representing greater similarity. However, the correlation coefficient is only sensitive to shape and not to scatter and elevation. The correlation between two profiles with the same shape but different elevation will be 1.0. This loss of information when using the correlation coefficient as a similarity measure can lead to misleading conclusions. Distance measures are sensitive to all three of the profile characteristics. Distance coefficients range from 0 to infinity with larger values indicating greater distance or dissimilarity between two objects. 
One of the most commonly used distance measures is Euclidean distance which is the square root of the sum of the squared differences of the $p$ variables for cases $\underline{i}$ and $j$. To avoid the square root term, the coefficient is often squared and expressed as "squared Euclidean distance." Other popular distance metrics include Manhattan distance, or city-block metric, which is the summation of the absolute values of the variable differences, and Mahalanobis distance which removes the correlation between the observed variables. Both distance and similarity metrics have been used in cluster analysis procedures but distance coefficients are generally preferred. The choice of a distance or a similarity metric would depend on whether or not the researcher wants to distinguish profiles only by shape or if all three characteristics are to be taken into consideration.

The next step in a cluster analysis is the process of grouping similar individuals. There are a host of clustering algorithms available. In the social sciences, hierarchical agglomerative algorithms are generally used. These methods begin with $\underline{N}$ clusters and sequentially merge the most similar cases until all individuals are contained in one cluster. The sequence of hierarchical mergers can be represented visually by a tree diagram called a dendrogram. Once individuals are assigned to a cluster, they cannot be removed at a later step. This creates nonoverlapping, nested clusters. The hierarchical clustering methods are distinguished by their different rules for determining which mergers occur at each step. Some of the most popular hierarchical clustering methods include: single linkage, complete linkage, average linkage, and Ward's method. Simulation studies 
by a number of researchers (Milligan 1981a; Milligan \& Cooper, 1987; Edelbrock, 1979; Blashfield, 1976) have demonstrated Ward's method to be the most accurate of these algorithms at recovering the correct cluster structure.

With hierarchical agglomerative cluster methods, all possible solutions between $\underline{N}$ the number of individuals, and 1, all individuals in one cluster are presented. This creates the problem of how to determine the optimal number of clusters for a data set. Although this is a critical decision, there is no generally accepted solution. A number of different approaches to solving the number of clusters problem exit. The dendrogram or tree diagram can be used to visually determine at what point dissimilar clusters start to merge. Theoretical assumptions can be a guide to determining how many clusters are expected. Several cluster solutions may be examined to find a solution which contains the most interpretable profiles. There are also a number of mathematical stopping rules that have been developed to find the optimal cluster solution. Milligan and Cooper (1985) conducted a simulation study on 30 different stopping rules and found the pseudo $\mathrm{F}$ test and the pseudo $\mathrm{t}^{2}$ to be the most accurate. These two stopping rules along with the Cubic Clustering Criterion (Sarle, 1983), have been incorporated into the SAS cluster analysis procedure. If the data set is large enough, splitting it into two samples allows for replication of the cluster pattern solutions. Replication can also be performed by using different clustering methods. Similar profiles found for the same number of clusters across samples or across methods, provide strong support for the cluster solution. Since there is no consensus on how to find the number of 
clusters, the researcher is advised to employ several of these options when determining the optimal number of clusters.

Interpreting the identified clusters involves examining the characteristics of the cluster profiles. Cluster profiles are created from the mean values of the $p$ variable for each of the $\underline{M}$ clusters. The variables in the profile are standardized so that all of the variables can be displayed with the same metric and each variable is given equal weight in creating the profile. For example, a T-score standardization (mean 50 and standard deviation 10) allows for easy interpretation of the magnitude of differences among the variables because a one point difference indicates a tenth of a standard deviation. The profile properties described earlier (elevation, scatter, and shape) are used to describe the standardized cluster profiles.

Validation of the $\underline{M}$ clusters is a critical step that can be accomplished through replication of the clusters and through significance testing with relevant external variables. Cross-validation replication is an empirical approach to replicating a cluster solution across samples (Breckenridge, 1989; Humphreys \& Rosenheck, 1995; Morey, Blashfield, \& Skinner, 1983). In this procedure the data in Sample A is clustered. The data points in Sample B are matched to the nearest cluster centroids of Sample A. Cluster analysis is then performed on Sample B. Replication is assessed as the amount of agreement between the two partitions of Sample B. The same procedure can also be conducted on Sample A for double cross-validation replication.

For external validation the $\underline{M}$ clusters become the levels of an independent categorical variable and the validating variables are treated as dependent variables. The 
external validating variables should be of theoretical relevance to the variables in the cluster analysis. Meaningful group differences found between the $\underline{M}$ clusters provide evidence of external validity for the cluster solution. Establishing both stability and external validity is important evidence of the verisimilitude of the clusters.

\section{The Present Study}

In the present study, cluster analysis and cluster validating procedures were proposed as a means of examining hypothesized construct relationships within the TTM for exercise behavior. Cluster analysis was used to find groups of exercisers differentiated by the TTM's multivariate outcome space defined by the Criterion Measurement Model (Velicer et al., 1996). Several possible outcomes of the exercise typology were hypothesized, all of which have implications for the application of the TTM to exercise behavior.

\section{Possible Cluster Analysis Outcomes}

No discernible clusters. The sample of individuals may not contain different groups of exercisers and may best be characterized as a homogeneous sample based on the outcome space defined by decisional balance and confidence to exercise. This cluster solution would not provide evidence to support hypothesized TTM construct relationships.

Clusters corresponding to the Stages of Change. Finding a typology of exercisers that corresponds with the Stages of Change would support the construct validity of the 
stages of change for exercise behavior. Such a typology would indicate that each cluster is defined by a profile of Pros, Cons, and Confidence that is the expected pattern for a particular Stage of Change. Predictions were made about what cluster profiles for each stage of change would look like. For Precontemplation, an inverted "V" profile was expected with the Pros of exercise low, the Cons of exercise high and Confidence to exercise low. A Contemplation cluster would have a flat profile with average elevation and little scatter. The Pros and Cons would be at approximately the same level with Confidence at a lower level. A Preparation profile was expected to have a shallow "V" profile with low scatter and average elevation. The Pros should be somewhat higher than the Cons and Confidence should be average. Regular exercisers, those in Action and Maintenance would most likely have similar cluster profiles. The shape is expected to be a distinct "V" with higher scatter than the Preparation profile. The Pros should be much higher than the Cons and Confidence should also be high.

A small number of clusters not related to the Stages of Change. This solution would indicate that there are alternative ways to group individuals that relate to changing exercise behavior but not related to the stages of change. Such a solution would challenge the applicability of the stages of change to exercise behavior as well as call into question hypothesized construct relationships between stages of change and other model constructs.

Clusters corresponding to stage subtypes. Although the cluster analysis was not done within stages, the existence of exercise stage subtypes was considered plausible if, instead of a one to one correspondence between stages and clusters, a limited number of 
clusters were found that were associated with adjacent stage groups. That is, if a cluster was associated with $\mathrm{PC}$ and $\mathrm{C}$ and not with $\mathrm{A}$ and $\mathrm{M}$, then that cluster would be considered a subtype group within the early stages of change. Finding subtypes within the stages of change for exercise would provide further evidence that subtypes within stages exist and that they generalize to health behaviors other than smoking.

A Large number of clusters not related to the stages of change. An alternative typology would be a large number of clusters that is meaningful but not a replication of the smoking subtype typology. Finding that a cluster is as equally likely to be associated with Precontemplation as it is with the Maintenance stage would not support the generalizability of stage subtypes across health behaviors.

\section{Hypotheses Regarding Cluster Validation}

To demonstrate the verisimilitude of a cluster typology, there must be evidence of its reliability and validity. Reliability can be demonstrated through replication procedures. Two replication procedures were employed in the present study: random subsample clustering, and cross-validation. Finding consistent results between these two tests was expected to be an indication of the reliability of the cluster solution. Statistical significance testing with relevant external variables was used to determine validity of the cluster solution. Hypotheses for the different external validation variable domains are as follows:

1. Based on the CMM, a cluster typology that differentiates the multivariate outcome variables of Pros, Cons, and Confidence, should have meaningful differences in 
exercise behavior. It was hypothesized that validation of any exercise cluster solution would require that the clusters were differentiated by exercise behavior.

2. It was hypothesized that an exercise cluster typology that related to the exercise stages of change should also have an association with the exercise processes of change. Cluster profiles expected of sedentary and less active individuals should, on average, be using the processes of change less than clusters of more active individuals. This is particularly true for the behavioral processes of change. Experiential process use may have a more curvilinear relationship across an exercise typology as demonstrated in previous studies (Marcus, Rossi, et aI. 1992; Prochaska et al., 1991).

3. It was hypothesized that a cluster typology should have some association with the stages of change for regular exercise. It was difficult to predict the degree of association as indicated by the possible outcomes hypothesized for the cluster solution.

4. Concise hypotheses about the relationship between the exercise clusters and stage of change for other risk factors were difficult to make. Smoking status has only shown modest or no relationship with physical activity (King et al., 1992, Dishman, 1990). A recent study of stage of change for physical activity, diet, and smoking in a sample of 8,000 members of a HMO found the correlation between stage of physical activity and stage of smoking was .10 , and the correlation between physical activity and fruit and vegetable intake was .32 (Boyle, O'Conner, Pronk, Tan, 1998). The relationship between stage of change for sun protection and exercise has not been published. Based on this previous research, a small effect was predicted for smoking, a small to medium 
effect was predicted for diet, and the assessment of the relationship between exercise and sun protection was considered exploratory.

5. Variables such as gender, age, BMI, education level, and perceived health have all been associated with exercise behavior to varying degrees (Dishman, 1990; King et al., 1992; Sallis \& Hovell, 1990). The most consistent associations for exercise behavior have been positive relationships with perceived health and level of education. These variables were predicted to be related to the exercise typology. 


\section{Method}

\section{Participants}

A sample of 362 adults between the ages of 18 and 75 completed a survey through a random telephone interview. Ten completed surveys were not delivered until after the analyses for the current study were nearly complete. Six participants were dropped from the analyses because of insufficient data bringing the sample size for the present study to 346. Complete demographic descriptive information about the sample is presented in Table 2 . The sample was $62 \%$ female, $95 \%$ white, with a median household income of between $\$ 30,000$ and $\$ 40,000$. US Census data from 1990 for Rhode Island estimated the state to be approximately $52 \%$ female, $92 \%$ white, with a median household income of $\$ 32,000$. Comparison with the census data indicated that the current sample had a higher than expected percentage of females and slightly higher percentage of whites. Employment and marital status were equivalent to that reported from a large representative sample of 4,144 smokers in Rhode lsland (Fava, Velicer, \& Prochaska; 1995).

Health related sample characteristics are presented in Table 3. The distribution for perceived general health was very similar to that of a sample of over 19,000 member of a New England managed care organization (LaForge, 1992). The remaining health status questions were compared with national statistics from the Centers for Disease Control (CDC) (1997, August), the Arthritis Foundation (1997, December), and the American Heart Association (AHA) (1997, December). Overall, the comparison with the national health statistics indicated that the current sample was comparable in health status 
to the general US population. The only notable exception was the lower percentage of smokers (19\%) in the current study. However, recent data from a worksite clinical trial of 1,906 individuals in Rhode Island and Massachusetts was also 19\% smokers (Laforge, 1998).

Table 4 presents distributions of individuals at each stage of change for exercise, smoking, diet, and sun protection. Comparison of stage distributions with other studies can be difficult for some of these behaviors because the methods of staging and the definitions of the target behaviors have varied and changed over time. Assessment of stage of change for cigarette smoking is the most well established, and the present study's distribution replicates other studies (Velicer, Fava et al. 1995). The target behavior for Diet was fruit and vegetable consumption. In other studies the target behavior has been fat reduction or increasing dietary fiber. Interestingly, the stage distribution for diet in the present study is very similar to the diet distribution found in the worksite clinical trial of 1,906 indiviuals (Laforge, 1998) where the target behavior was to avoid eating high fat foods. The stage distribution for regular exercise was compared with distributions reported by Reed et al. (1997) from three different samples. Clear similarities among the samples were not present. The largest discrepencies were related to the Maintenance stage of change which was usually between $20 \%$ and $40 \%$ rather than the $50 \%$ found in the present study. Clear stage of change distribution similarities were also not found for Sun Protection behavior in comparision with the large managed care sample and the worksite sample mentioned previously. 
Table 2

Sample Characteristics: Demographics

Variable

Total Sample

Gender

females

$212(62 \%)$

males

$132(38 \%)$

Age (mean)

(s d)

15

Race

white

$328(95 \%)$

$8(2 \%)$

black

$11(3 \%)$

other

$\$ 30-\$ 40 \mathrm{~K}$

Household Income (median)

14

Education (median grade level)

Marital Status

married

$195(56 \%)$

not married

$67(19 \%)$

divorced

$38(11 \%)$

living with partner

$22(6 \%)$

separated

$6(2 \%)$

widowed

$18(5 \%)$

Employment Status

employed for wages

$209(60 \%)$

self-employed

$33(10 \%)$

student

$18(5 \%)$

out of work

$18(5 \%)$

homemaker

$23(7 \%)$

retired

$46(13 \%)$ 
Table 3

Sample Characteristics: Health Related

Variable $\quad$ Present Study $\quad$ Regional \&

National

Percentages

Perceived General Health

$\begin{array}{lrc}\text { excellent } & 75(21 \%) & 20 \%^{a} \\ \text { very good } & 150(43 \%) & 40 \% \\ \text { good } & 99(28 \%) & 32 \% \\ \text { fair } & 24(7 \%) & 7 \% \\ \text { poor } & 4(1 \%) & 1 \%\end{array}$

Specific Health Problems (\% Yes)

$\begin{array}{lrc}\text { diabetes } & 6.0 \% & 4.6 \%^{\mathrm{b}} \\ \text { arthritis } & 15.3 \% & 14.3 \%^{\mathrm{c}} \\ \text { high blood CHL } & 18.2 \% & 20.6 \%^{\mathrm{b}} \\ \text { high blood pressure } & 19.3 \% & 23.1 \%^{\mathrm{b}} \\ \text { heart attack stroke } & 3.4 \% & 6.6 \%^{\mathrm{d}} \\ \text { any form of cancer } & 5.1 \% & 3 \%\end{array}$

Body Mass Index (mean, sd)

fermales with $\mathrm{BMI} \geq 27.3$

$26.2(4.7)$

males with $\mathrm{BMI} \geq 27.8$

$21.4 \%$

$21.6 \%^{b}$

$29.0 \%$

$28.6 \%^{b}$

Current Smokers

$19 \%$

$24.7 \%^{b}$

\footnotetext{
${ }^{2}$ Assessed from a sample of over 19,000 members of a New England managed care organization. ${ }^{\mathrm{b}}$ Estimates for the state of Rhode Island from the CDC Surveillance

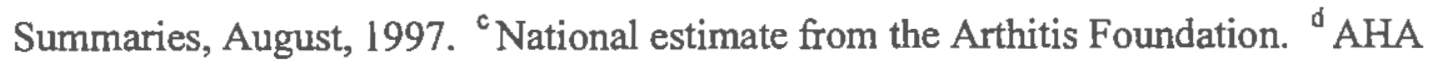
national estimate in 1994.
} 
Table 4

Stages of Change for Health Behaviors

\begin{tabular}{|c|c|c|c|c|}
\hline Stage of Change & Exercise & $\begin{array}{l}\text { Cigarette } \\
\text { Smoking }\end{array}$ & $\operatorname{Diet}^{\mathrm{a}}$ & $\begin{array}{c}\text { Sun } \\
\text { Protection }^{b}\end{array}$ \\
\hline Precontemplation & $\begin{array}{c}46 \\
(13 \%)\end{array}$ & $\begin{array}{c}26 \\
(7 \%)\end{array}$ & $\begin{array}{c}108 \\
(30 \%)\end{array}$ & $\begin{array}{c}106 \\
(30 \%)\end{array}$ \\
\hline Contemplation & $\begin{array}{c}27 \\
(8 \%)\end{array}$ & $\begin{array}{c}24 \\
(7 \%)\end{array}$ & $\begin{array}{c}36 \\
(10 \%)\end{array}$ & $\begin{array}{c}20 \\
(6 \%)\end{array}$ \\
\hline Preparation & $\begin{array}{c}76 \\
(22 \%)\end{array}$ & $\begin{array}{c}18 \\
(5 \%)\end{array}$ & $\begin{array}{c}91 \\
(26 \%)\end{array}$ & $\begin{array}{c}72 \\
(21 \%)\end{array}$ \\
\hline Action & $\begin{array}{c}26 \\
(7 \%)\end{array}$ & $\begin{array}{c}14 \\
(4 \%)\end{array}$ & $\begin{array}{c}9 \\
(3 \%)\end{array}$ & $\begin{array}{c}24 \\
(7 \%)\end{array}$ \\
\hline Maintenance & $\begin{array}{c}174 \\
(50 \%)\end{array}$ & $\begin{array}{c}103 \\
(30 \%)\end{array}$ & $\begin{array}{c}107 \\
(31 \%)\end{array}$ & $\begin{array}{c}124 \\
(36 \%)\end{array}$ \\
\hline Never smoked & & $\begin{array}{c}166 \\
(47 \%)\end{array}$ & & \\
\hline
\end{tabular}

Note: a. Diet target behavior was 5 servings of fruits and vegetables per day.

b. Sun Protection target behavior was consistently using sunscreen with an SPF rating of at least 15 . 


\section{Procedure}

Telephone interviews were conducted by the Cancer Prevention Research Center's survey center between May 15 and July 15, 1997. Telephone numbers were obtained from the Rhode lsland telephone directory for Providence and surrounding vicinity which included areas of Southern Massachusetts. Phone numbers were selected by taking every 25th telephone number from the directory. Individuals between the ages of 18 and 75 were eligible to participate in the study. Interviews were conducted anonymously and no incentives were offered to individuals for completing the survey. If more than one eligible person resided in a household an interview was requested with the individual who would next celebrate a birthday.

Telephone interviewers explained that they were from the Cancer Prevention Research Center at the University of Rhode Island and that they were conducting a study to learn about peoples' behaviors and attitudes regarding exercise. If an individual agreed to take part in the study, a confidentiality statement was read by the interviewer. The statement assured participants that all information was strictly anonymous, confidential, and for research purposes only. An interview usually took between 20 and 30 minutes to complete.

529 eligible participants were contacted, of which 130 (24.6\%) individuals refused to participate, $17(3.2 \%)$ individuals started the survey but refused to continue and were not agreeable to completing the survey at a later date. Twenty (3.8\%) individuals were determined to be eligible but a gate keeper refused access to the individual. An additional 145 homes were called in which a gate keeper refused and it 
was not determined if an eligible individual lived in the home. Thirty-four interviews were started but never completed. A Total of 362 participants completed the survey. A response rate of $68.4 \%$ was computed by dividing the number of completed surveys by the sum of completes, refusals, incomplete refusals and gate-keeper refusals.

\section{$\underline{\text { Measures }}$}

The questionnaire was designed to assess cognitions and behavior relating to participation in regular exercise. Measurement scales were based on the Transtheoretical model of change. These included stage of change for regular exercise, a decisional balance inventory, confidence to exercise regularly, and a processes of change inventory. Measures of exercise frequency and physical activity were also included. Demographic characteristics and health related characteristics were also assessed. A copy of the questionnaire is included in the Appendix.

Part of the purpose of the data collection, in addition to conducting the present study, was to develop new scales for three of the TTM constructs: Decisional Balance, Confidence, and Processes of Change. The goal was to create a set of TTM measures for exercise behavior that were not directly translated from the smoking cessation research and were developed on a general adult population. New scale items were generated, and along with items from the previously developed inventories (Marcus, Rakowski, et al., 1992; Marcus, Selby, et al., 1992; Marcus, Rossi, et al. 1992), were rated by experts to assess face validity. Items with consistently high ratings were included in the inventories used in the present study. Some of the measurement development work is reported here, 
but a complete description is reported elsewhere (Rossi, Norman, Nigg, \& Benisovich, 1998).

Stages of Change for Regular Exercise. The five stages of change

(Precontemplation, Contemplation, Preparation, Action, Maintenance) were assessed with a four question algorithm. Participants were first read a definition of regular exercise which included several examples (see Appendix). The questions were answered with response choices YES or NO. Skip patterns within the series of questions determined which questions participants received based on their previous response. Figure 1 displays how question response patterns grouped individuals into the five stages of change.

The staging categories were considered "intention based" because intention to exercise regularly differentiated the first three stages of change. For example, Precontemplation was defined as not exercising regularly and not intending to exercise regularly in the next six months; Contemplation was defined as not exercising regularly but seriously considering exercising regularly in the next six months, and Preparation was defined as not exercising regularly but seriously considering exercising regularly in the next thirty days (see Figure 1). This conceptualization of the stages of change for exercise is somewhat of a departure from the stage definitions employed by Marcus and colleagues (Marcus, Selby et al, 1992). Their staging definition defines Preparation based only on current behavior. That is, individuals are classified into Preparation if they are exercising some but not regularly. Intention to exercise regularly for $\mathrm{PC}$ and $\mathrm{C}$ is defined as "not in the next six months" and "in the next six months" respectively, but 
these stages are further defined by "not exercising regularly and not exercising even some of the time."

The questions pertaining to stage of change in the questionnaire (see Appendix) allowed for both the intention based staging and the staging used by Marcus and colleagues to be calculated for the sample. After comparing the distributions of both methods of staging, it was decided to focus on the intention based staging for a number of reasons. The intention based staging was simpler, it was closer to the original conceptualization of the stages of change (Prochaska \& DiClemente, 1983), and it created a staging distribution with more individuals in the Precontemplation and Contemplation stages. Table 5 displays the relationship between the two methods of staging. The Action and Maintenance stages do not vary but there is a shift from PC and $\mathrm{C}$ to $\mathrm{PR}$ when going from intention based staging to how Marcus, Selby et al. (1992) defined the stages of change. For further analyses intention based staging will be called exercise stages of change.

Decisional Balance. The Decisional Balance Inventory consists of two constructs which involve cognitive and motivational aspects of human decision-making (Prochaska et al., 1994; Velicer et al., 1985). These constructs have been labeled the "Pros" and the "Cons" of change. Marcus, Rakowski, and Rossi (1992) developed a 16 item Decisional Balance inventory for exercise. Internal consistency for the 10 item Pros scale and the 6 item Cons scale was .95 and .79 respectively.

In the present study, the Pros and Cons of exercise scales were derived from an inventory of 49 items representing the eight domains of decision making theorized by 
Janis and Mann (1977). These domains are grouped into four main categories: (1) utilitarian gains and losses for self; (2) utilitarian gains and losses for others; (3) selfapproval or self-disapproval; and (4) approval or disapproval from significant others. Several items were included from the Decisional Balance Inventory for Exercise developed by Marcus, Rakowski, and Rossi (1992). Participants responded to each on using a 5-point Likert scale with written anchors of 1 "not at all important" to 5 "extremely important". Item reduction using Principal Components Analysis (PCA) resulted in two 10 item components accounting for $43.5 \%$ of the total item variance. Table 6 contains the items retained for the Pros and Cons scales and their component loadings. The average loading of an item on its corresponding component was .641 . Internal consistency, measured with coefficient alpha for the Pros and Cons was .91 and .72 respectively. Raw score descriptive statistics for the summated scales are presented in Table 7. The correlation between the Pros and the Cons scales was $r=.06$, indicating that these scales were orthogonal. This two factor uncorrelated measurement structure for decisional balance is a replication of the structure found for decisional balance for smoking cessation (Velicer, et al. 1985) and exercise behavior (Marcus, Rakowski, et al., 1992).

Exercise Confidence. Bandura $(1977,1982)$ defined self-efficacy as the confidence one has to perform a particular behavior. Velicer et al. (1990) identified a hierarchical measurement structure for self-efficacy related to smoking cessation consisting of three constructs labeled Positive/Social, Negative/Affective, and Habit/Addictive. Confidence in one's ability to exercise regularly has been assessed with 
both unidimensional general measures of confidence (Marcus, Selby et al., 1992; Sallis et al., 1988) and with multi-dimensional situational measures of confidence (Benisovich et al., 1998). The general confidence measure consists of the five items representing adverse conditions such as being tired, in a bad mood, on vacation, not enough time, and when it is raining or snowing. Marcus and Owen (1992) reported internal consistency of the five item scale to be above .80 in two different samples.

In the present study, a 25 item inventory was administed to participants in an attempt to replicate the multi-dimensional structure for exercise confidence found by Benisovich et al. (1998). Participants responded to each item on a 5-point Likert scale ranging from 1 "Not at all confident" to 5 "Extremely confident" (see Appendix). Velicer et al. (1990) demonstrated that distinguishable factors can be disguised as an artificial "general factor" when a sizable number of participants respond to an entire question inventory with all ' 1 ' or all ' 5 ' response choices. This pattern of responding is called an extremity response style and can occur in a heterogeneous sample where there are groups of individuals who are either completely confident across all situations or completely unconfident across all situations. Non-normality with a very high frequency of scores in the tails of the distribution would be an indication of extremity response style for the inventory. The mean scores for the 25 confidence items in the present study were esentially normally distributed with a skewness of -.038 and a kurtosis of.- .707 , indicating no evidence of extremity response style.

A PCA on the matrix of correlations was conducted and both the MAP rule (Velicer, 1976) and parallel analysis (Lautenschlager, 1989) indicated a two component 
solution. Interpretation of the two components revealed a component representing primarily internal states and feeling relating to confidence, and a second component representing external situations. The correlation between the two scales was 64 .

Because a single scale for Confidence was needed for cluster analysis, ten items were selected from the two scales to form one scale of global confidence. A final PCA on the ten retained items extracting one component accounted for $43.6 \%$ of the total variance with an average component loading of .657. Internal consistency for this scale was .86 . Table 8 displays the ten confidence items and their component loadings.

Processes of Change. The Processes of change are affective, cognitive, and behavioral activities that people use as they change their behavior. The original 40 item Processes of Change Inventory measures ten processes and was developed for smoking behavior (Prochaska, Velicer, DiClemente, \& Fava, 1988). External validity for these scales has been demonstrated in both cross-sectional (Prochaska \& DiClemente, 1983; DiClemente et al. 1991; Fava et al., 1995) and longitudinal studies (Prochaska, Velicer, DiClemente, Guadagnoli, \& Rossi, 1990; Prochaska, et al., 1991). A 39 item questionnaire assessing these same processes was developed for exercise behavior (Marcus, Rossi et al., 1992). Internal consistency for the exercise processes of change ranged from .62 to .89 across the 10 scales.

In the present study, a 68 item inventory was administered to participants to assess processes of change for regular exercise. These items were a final pool of items that resulted from a large set of newly generated items and the 39 items from the Marcus, Rossi et al. (1992) processes of change for exercise inventory. This initial larger pool of 
items was rated by individuals knowledgeable in the TTM in regard to how well each item represented one of the ten processes of change. The purpose of developing a new inventory for exercise processes of change was to create a measure in which the process items were not directly translated from the smoking inventory developed by Prochaska et al. (1988).

Confirmatory factor analysis using LISREL software was used to reduce the number of items in the 68 item inventory and assess the fit of the hypothesized 10 factor model to the data. Modification indices provided in the LISREL software were used to identify complex items in an effort to maximize the discriminant validity of the 10 factors. The inventory was reduced to 40 items. The fit of a correlated 10 factor model to the data was good as indicated by structural equation modeling diagnostic fit indices $\left(\chi_{(695)}^{2}=1628.4, \chi^{2} / \mathrm{df}=2.34, \mathrm{CFI}=.849, \mathrm{AASR}=.046\right)$. Table 9 displays the final 40 item inventory with each item's estimated standardized loading from the 10 correlated factors model. A more complete description of the measurement development of the processes of change scales can be found in Rossi et al. (1998). Table 10 displays the summary statistics, including coefficient alphas and pearson correlation coefficients for the summated processes of change scales.

Self-Reported Exercise and Physical Activity Behavior. Exercise and physical activity behavior were assessed with two self report measures. Together these two measures provide information about daily physical activity related to occupation and home life, as well as planned leisure time exercise activity. 
Leisure time exercise was assessed with the Godin's Leisure Time Exercise Questionnaire (GLTEQ) (Godin \& Shepard, 1985; Godin, Jobin, \& Boullon, 1986). With this measure, individuals are asked to consider a typical week and indicate the number of times per week they engaged in strenuous, moderate, and mild forms of exercise for at least 20 minutes in duration. Examples of each type of exercise are provided. Godin et al. (1986) demonstrated two week test-retest reliability to be .64 and statistically significant concurrent validity coeffiecients with $\mathrm{VO}^{2} \max$, body fat, and muscular endurance. The GLTEQ has the advantages of being brief and easy to understand and has been shown to have the strongest association with stages of change (Buxton, Wyse, \& Mercer, 1996).

The second assessment was a physical activity measure taken from the Paffenbarger/ Harvard Alumni Questionnaire (P/HAQ) (Paffenbarger, Blair, Lee, \& Hyde, 1993). Individuals are instructed to indicate how much time they spend on a usual weekday and weekend day engaging in vigorous, moderate, light, sitting, and sleeping or reclining activities. The total number of hours/day should sum to 24 hours. In the present study, weekday and weekend day means for the five activity types did sum to nearly 24 hours (see Table 11).

Validity for the $\mathrm{P} / \mathrm{HAQ}$ has been demonstrated by its inverse relationship with incidence of disease states, and correlations with high-density lipoprotein (positive) and body mass index (negative) (Lee, Hsich, \& Paffenbarger, 1993; Washburn, Smith, Goldfield, \& McKinlay, 1991). Test-retest reliability has ranged from .50 to .76 across a number of studies (Montoye, Kemper, Saris, \& Washburn, 1996). 
Stages of Change for Health Behaviors. Stage of change was assessed for cigarette smoking, diet, and sun protection. Stage of change for smoking was assessed with a six question algorithm which has been used in previous studies (DiClemente et al., 1991; Fava et al. 1995). Stage of change for diet asked participants, "Do you eat at least 5 servings of fruits and vegetables per day?" (Laforge, Greene, \& Prochaska, 1994). Stage of change for sun protection was assessed with the question, "Do you consistently use a sunscreen with an SPF of 15 or more whenever you know you will be out in the sun for more than 15 minutes?"' (Rossi, Blais, Redding, \& Weinstock, 1995). These two questions were each followed by five response choices which corresponded to one of the five stages of change.

Demographics. Several questions were asked that assessed general demographics and health related participant characteristics. General demographics included: gender, level of education, age, marital status, and race. Height and weight were assessed to calculate body mass index (BMI). Assessment of health characteristics included perceived health and the presence of several disease states (diabetes, arthritis, hypertension, elevated blood cholesterol, heart attack or stroke, and cancer). 
Table 5

Relationship Between Intention Based Staging and Marcus Based Staging

Intention Based Staging

(frequency count)

\begin{tabular}{|c|c|c|c|c|c|c|}
\hline $\begin{array}{l}\text { Marcus } \\
\text { based } \\
\text { Staging }\end{array}$ & $\mathrm{PC}$ & C & PR & $\mathrm{A}$ & M & Row Total \\
\hline $\mathrm{PC}$ & 17 & & & & & 17 \\
\hline $\mathrm{C}$ & & 5 & 12 & & & 17 \\
\hline PR & 24 & 22 & 63 & & & 109 \\
\hline A & & & & 26 & & 26 \\
\hline $\mathbf{M}$ & & & & & 172 & 172 \\
\hline $\begin{array}{l}\text { Column } \\
\text { Total }\end{array}$ & 41 & 27 & 75 & 26 & 172 & 341 \\
\hline
\end{tabular}


Table 6

Items and Component Loadings for the Decisional Balance for Exercise Scale

Scale Item

Component

Loading

Pros of Exercise

I would have more energy for my family and friends if I exercised regularly.

Others would have more respect for me if I exercised.

I would decrease my chance of having a heart attack if I exercised.

.609

It would be easier for me to perform routine physical tasks if I exercised regularly.

Exercising puts me in a better mood for the rest of the day.

Exercise would lower my anxiety.

I would have more self respect if I exercised.

Regular exercise would help me have a more positive outlook on life.

I would look better if I exercised.

Exercise allows me to participate in activities with my friends

\section{Cons of Exercise}

Exercise clothing and equipment is too costly.

Exercise prevents me from spending time with my friends.

Regular exercise would take too much of my time.

I would feel out of place in an exercise group.

I am too out of shape to exercise.

I think I would be too tired to do my daily work after exercising.

There is too much I would have to learn to exercise.

I feel uncomfortable when I exercise because I get out of breath and my heart beats very fast.

I am afraid to find that $I$ am not good at exercising.

Exercise is too boring to do it regularly. .566

Note. * Indicates an item from Marcus et al. 1992. 
Table 7

Raw Score Descriptive Statistics for Pros, Cons, and Confidence Summated Scales

Raw Score Descriptive Statistics

\begin{tabular}{lccccc}
\cline { 3 - 5 } Variable & mean & $s \mathrm{~d}$ & alpha & skewness & kurtosis \\
\hline Pros & 32.43 & 9.68 & .91 & -.409 & -.511 \\
Cons & 15.80 & 5.21 & .72 & 1.23 & 1.82 \\
Confidence & 29.48 & 8.57 & .86 & -.082 & -.618 \\
\hline
\end{tabular}

Note. Range for all summated scales was: $\min =10$ and $\max =50$. 
Table 8

Items and Component Loadings for the Exercise Confidence Scale

Scale Item

Component

Loading

I am confident that I can participate in regular exercise when...

I am tired.

$.691 *$

I am in a bad mood.

I feel I don't have the time.

The weather is bad.

I am under a lot of stress.

I am feeling lazy,

I don't have access to exercise equipment.

I am spending time with friends or family who do not exercise.

I am travelling.

I have to exercise alone.

Note. * Indicates an item from Marcus, Selby et al. (1992) inventory. 
Table 9

Items and Factor Loadings for the 40 Item Exercise Processes of Change

Scale Item

Component

Loading

\section{Consciousness Raising}

I think about information from articles and advertisements on how to make exercise a

.635

regular part of my life.

I look for information related to exercise.

I find out about new methods of exercising.

I seek information about which exercises are best for me.

Dramatic Relief

I react emotionally to warnings about an inactive lifestyle.

I get upset when I see people who would benefit from exercise but choose not to exercise.

I am afraid of the consequences to my health if I do not exercise.

I get upset when I realize that people I love would have better health if they exercised.

\section{EnvironmentaI Reevaluation}

I feel I would be a better role model for others if I exercised regularly.

I realize that I might be able to encourage others to be healthier if I would exercise more.

I wonder how my inactivity affects those people who are close to me.

Some of my close friends might exercise more if I did.

\section{Social Liberation}

I find society changing in ways that make it easier to exercise.

I am aware of that communities are spending more money on recreation centers and bike paths.

I notice that more businesses are encouraging their employees to exercise by offering fitness centers. 
(Table 9 continued)

I have noticed that famous people often advertise the fact that they exercise regulariy.

Self Reevaluation

I get frustrated with myself when I don't exercise.

I feel more confident when I exercise regularly.

I think that because I value health I should value exercise.

I believe that regular exercise will make me a healthier, happier person.

\section{Helping Relationships}

I have a friend who encourages me to exercise when I don't feel up to it.

I have someone who provides feedback about my exercising.

I have a workout partner.

I have someone who encourages me to exercise.

\section{Reinforcement Management}

One of the rewards of regular exercise is that it improves my mood.

I do something nice for myself for making efforts to exercise more.

I congratulate myself after I exercise.

If I engage in regular exercise, I find that I get the benefit of having more energy.

Self Liberation

I tell myself I am able to keep exercising if I want to.

I tell myself that I can keep exercising if I try hard enough.

I make commitments to exercise.

I believe that I can exercise regularly

\section{Stimulus Control}

I keep things around my place of work (school) that remind me to exercise.

I keep a set of exercise clothes conveniently located so I can exercise when ever I get the time.

I bring my athletic shoes to work so I will remember to take a walk at lunch time. 
(Table 9 continued)

I use my calander to schedule my exercise time.

Counter Conditioning

Instead of remaining inactive, I engage in some physical activity.

Rather than viewing exercise as another task to get out of the way, I try to use it as my .625 time to relax.

Instead of relaxing by watching TV or eating, I take a walk or exercise.

I think of exercising as fun, rather than as a burden.

Note. * Indicates an item from Marcus et al. (1992) inventory. 


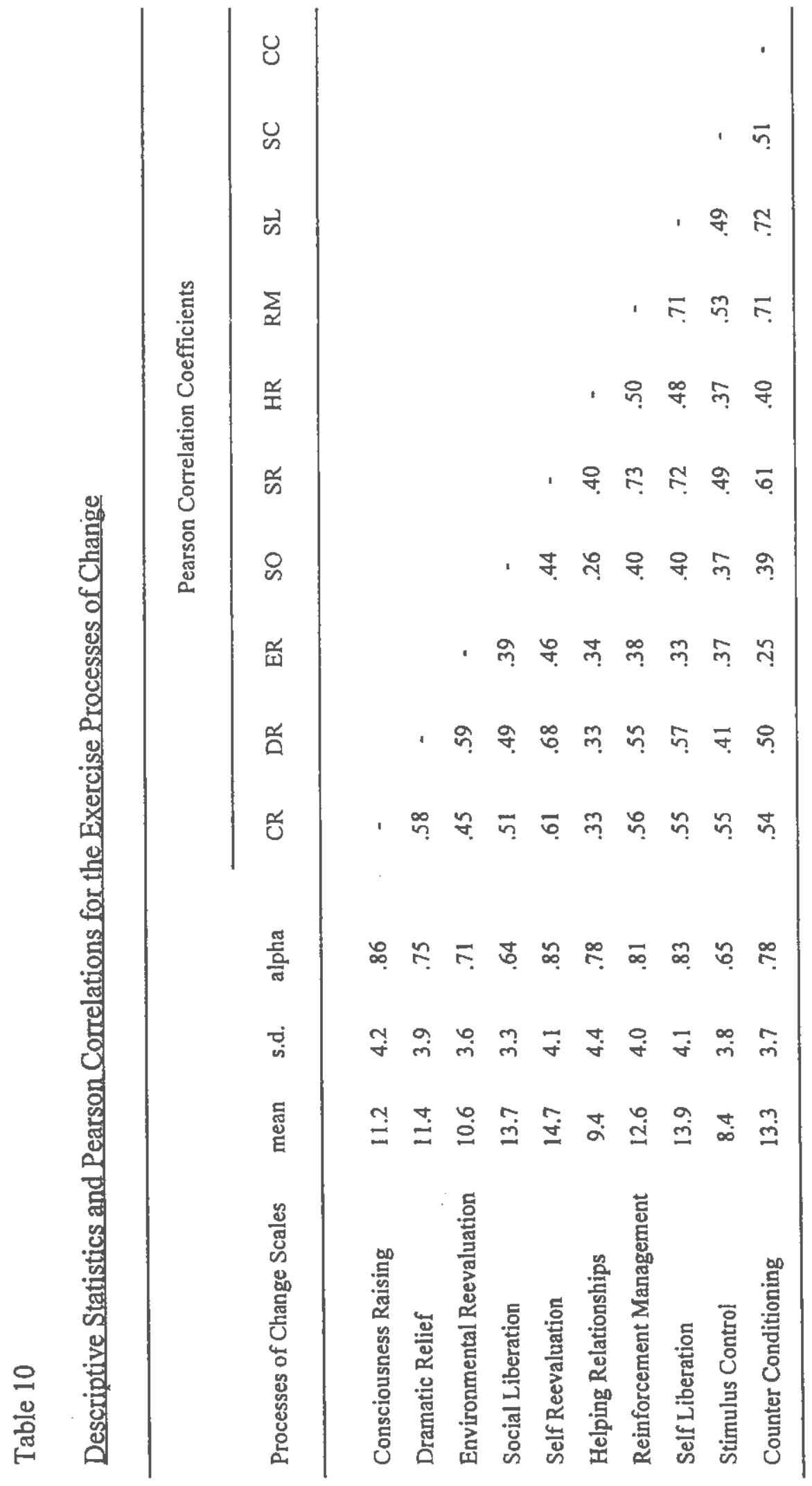


Table 11

Means and Standard Deviations from the Paffenbarger/Harvard Alumni Questionnaire

\begin{tabular}{|c|c|c|}
\hline \multirow[b]{2}{*}{ Type of Activity } & \multicolumn{2}{|c|}{$\begin{array}{l}\text { Hours Per Day } \\
\text { (Mean , S.D.) }\end{array}$} \\
\hline & Weekday & $\begin{array}{c}\text { Weekend } \\
\text { Day }\end{array}$ \\
\hline $\begin{array}{l}\text { Vigorous Activity } \\
\text { (e.g. strenuous sports, digging in the } \\
\text { garden, aerobic dancing, brisk } \\
\text { walking, heavy carpentry) }\end{array}$ & $\begin{array}{l}1.60 \\
(2.1)\end{array}$ & $\begin{array}{l}2.40 \\
(2.4)\end{array}$ \\
\hline $\begin{array}{l}\text { Moderate Activity } \\
\text { (e.g. housework, light sports, regular } \\
\text { walking, golf, yard work, painting, } \\
\text { ballroom dancing) }\end{array}$ & $\begin{array}{l}3.75 \\
(2.4)\end{array}$ & $\begin{array}{l}4.46 \\
(2.4)\end{array}$ \\
\hline $\begin{array}{l}\text { Light Activity } \\
\text { (e.g. office work, driving a car, } \\
\text { strolling, personal care) }\end{array}$ & $\begin{array}{l}6.27 \\
(3.4)\end{array}$ & $\begin{array}{l}4.79 \\
(2.5)\end{array}$ \\
\hline $\begin{array}{l}\text { Sitting Activity } \\
\text { (e.g. eating, reading, desk work, } \\
\text { watching tv, listing to the radio) }\end{array}$ & $\begin{array}{l}4.37 \\
(2.5)\end{array}$ & $\begin{array}{l}4.35 \\
(2.3)\end{array}$ \\
\hline Sleeping or Reclining & $\begin{array}{l}7.22 \\
(1.6)\end{array}$ & $\begin{array}{l}7.44 \\
(1.8)\end{array}$ \\
\hline
\end{tabular}




\section{Stages of Change}

Do you exercise regularly?

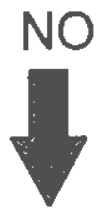

Are you seriously considering exercising regularly in the next 6 months?

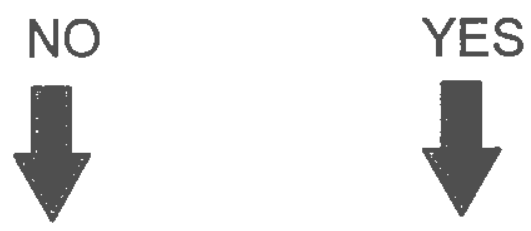

Precontemplation

Are you seriously considering exercising regularly in the next 30 days?

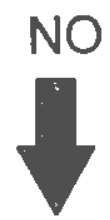

YES

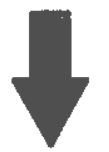

Contemplation

Preparation
YES

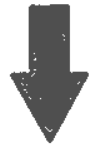

Have you been exercising regularly for the past six months?

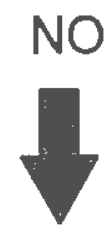

Action
YES

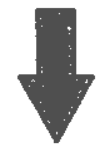

Maintenance

Eigure 1. Flow chart depicting the stages of change algorithm. 


\section{Results}

The results are presented as a series of steps and analyses. The first section describes the preliminary analysis which included data processing and decisions made prior to using cluster analysis. The second and third sections present the cluster analysis followed by a description of the process of interpreting the clusters. The fourth and fifth sections present internal validation analyses of the cluster solution patterns. The last five sections are each separate studies conducted to test the external validation of the clusters with relevant variables.

\section{Preliminary Analysis}

Variable Selection. The variables included in the cluster analysis were based on an outcome measurement model proposed by Velicer, Rossi, Prochaska, and DiClemente (1996). This model includes three constructs Positive Evaluation Strength, Negative Evaluation Strength, and Habit Strength, which have relevance across the Stages of Change. Positive Evaluation Strength and Negative Evaluation Strength were measured using the Pros of Exercise Scale and the Cons of Exercise Scale respectively. In place of Habit Strength, a scale measuring confidence to exercise regularly was be used.

Correlations among the three variables were small to moderate. The pros and cons were correlated at $r=.06$, the correlation between pros and confidence was $r=.41$, and between cons and confidence was $r=-.31$. The range of these correlations were considered appropriate for cluster analysis because the variables only shared a small 
amount of overlapping variance indicating that each could contribute to the cluster analysis.

Standardization of Variables. The variables used in the cluster analysis were standardized T-scores $($ mean $=50$, standard deviation $=10)$. Standardization was computed across the entire sample. Standardization serves both to equalize the contribution of each variable in the analysis and to put the variables on a comparable metric.

Assessment of Outliers. Box plots and bivariate scatter plots were used to identify outliers. Cases above or below three standard deviations on the pros, cons, or confidence scales were dropped from the analysis. This criteria lead to three cases being excluded from further analysis.

Splitting the Sample. The total sample was split into two subsamples by separating participants by even and odd number identification numbers. This resulted in Subsample 1 containing 177 individuals and Subsample 2 containing 165 individuals. Splitting the sample allowed for conducting separate cluster analyses and comparing the cluster solutions as a test of cluster replication.

\section{Cluster Analysis}

Distance Metric and Clustering Algorithm. The squared Euclidean distance metric and Ward's minimum variance clustering algorithm were used for all analyses (Ward, 1963). Euclidean distance metric was chosen because it is particularly sensitive to the cluster profile characteristics of shape, level, and scatter among the variables 
(Cronbach \& Gleser, 1953; Edelbrock, 1979). Ward's method of clustering is a hierarchical agglomorative procedure that tends to find spherical clusters. It has been shown to be one of the better clustering methods in several simulation studies (Blashfield, 1976; Milligan, 1980; Milligan \& Cooper, 1987).

Assessment of Ties. It is important to assess the influence of cluster ties which can occur at each step of the agglomorative clustering. Too many ties, or ties at the later steps of the analysis can result in inconsistent cluster solutions. At each step of the cluster analysis, the two subjects, or clusters, with the smallest distance between them are merged. If more than one merger is possible during a step because of equal minimal distance then the tie is broken with an arbitrary rule. SAS and SPSS have different rules for settling distance ties. If ties occur at the early steps of the cluster analysis and are relatively few, then the later steps, which are of interest, are usually unaffected by the ties. The SAS/STAT User's Guide release 6.03 (1988) contains a set of macros which assesses the influence of ties on a cluster solution. The SAS macros create a series of permutations of the data and cluster each data permutation. The consistency of participants' cluster membership for a cluster solution of interest is output. Fifteen permutations of Ward's method clustering were run for cluster solutions 8 through 3 . The results indicated that distance ties did not affect the cluster solutions for the sample as a whole or the two subsamples.

Determining the Number of Clusters. Once the cluster analysis was performed on the two subsamples using Ward's method and squared Euclidean distance the optimal cluster solution was determined. Since there is no definitive procedure for determining 
the number of clusters several methods were used. As a first step to narrow the range of cluster solutions that would be examined, the hierarchical cluster dendrograms were interpreted along with three stopping rule procedures: the pseudo F, pseudo $\mathrm{t}^{2}$, and cubic clustering criteria (CCC) (Calinski \& Harabasz, 1974; Milligan \& Cooper, 1985; Sarle, 1983). The dendrogram for subsample 1 indicated a five cluster solution, while the dendrogram for subsample 2 indicated a six cluster solution (see Figure 2). The pseudo F and pseudo $t^{2}$ tests both indicated six cluster solutions for both subsamples. The cubic clustering criteria (CCC) did not indicate a clear solution in either subsample. Because these methods for determining the number of clusters are not absolute, the second step involved examining cluster pattern replication between the two subsamples.

Cluster pattern replication was conducted by comparing the mean variable patterns for different cluster solutions between subsample 1 and subsample 2. First, mean standardized scores were plotted for the pros, cons, and confidence variables for cluster solutions 3 through 8 . Next, the pattern characteristics of profile shape, elevation, and scatter for the different solutions were compared (see Figures 3 though 8 ). The 3 cluster solutions contained profiles with somewhat similar shapes but differences in scatter indicated that this was a less than optimal solution (see Figure 3). The 4 cluster solutions did produce patterns that replicated well between the subsamples (see Figure 4). However, these solutions combined clusters with dissimilar patterns that were distinct in the 5 cluster solutions. The 5 cluster solutions generated four distinct pattern profiles that clearly replicated between the subsamples (see Figure 5). However, there was one cluster that was unique to each of the subsample solutions that was not replicated in the other 
solution. The unique profiles are indicated by the dotted lines in Figure 5 . The 6 cluster solutions also revealed similar profile patterns to the 5 cluster solution. It is at the 6 cluster solution where cluster profiles began to emerge which were similar in shape and scatter with only small differences in profile elevation. Arrows point to these clusters in Figure 6. Replication for the 7 and 8 cluster solutions was poor with each subsample solution containing several unique cluster profiles (see Figures 7 and 8).

Looking across the cluster profile patterns of solutions 3 through 8 , it was determined that solutions 3 and 4 contained some clusters that combined groups that appeared distinct in higher number solutions. On the other hand, cluster solutions beyond the five cluster solution only generated variations on existing cluster patterns that differed in terms of elevation. For solutions above seven clusters, the number of individuals per cluster was becoming too low to have a representative number of individuals within each cluster for further analyses. The 5 cluster solution was determined to be optimal because all of the pattern profiles were distinct and, four of the five patterns replicated between the two subsamples. Because the two patterns that did not replicate between the subsamples in the 5 cluster solution differed in shape, elevation, and scatter, they remained as separate clusters when the subsamples were combined for further analyses. Thus, the final number of clusters was six based on the 5 cluster solution.

Recombining the two subsamples. For further analyses the two subsamples were combined. This was necessary to have sufficient cell sizes and statistical power for further analyses. 
Subsample $1(n=165)$

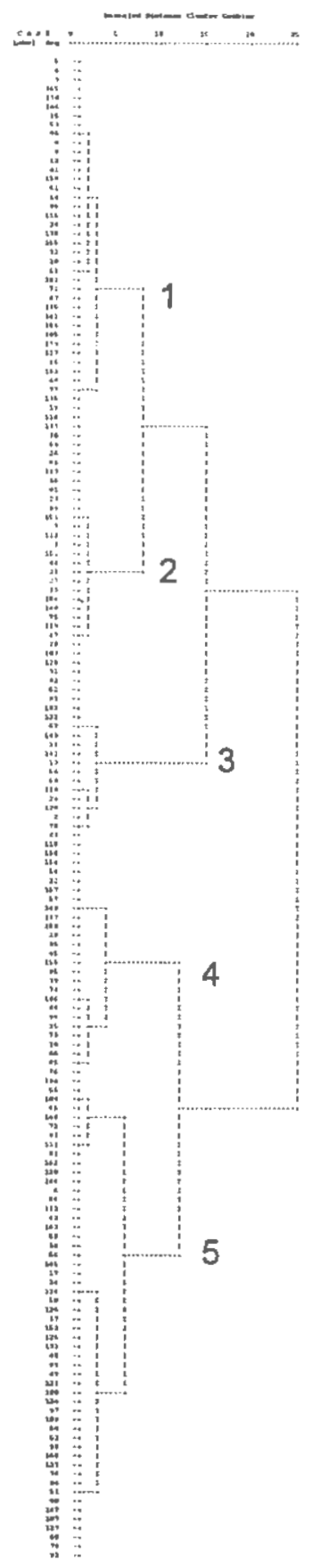

Subsample $2(n=177)$

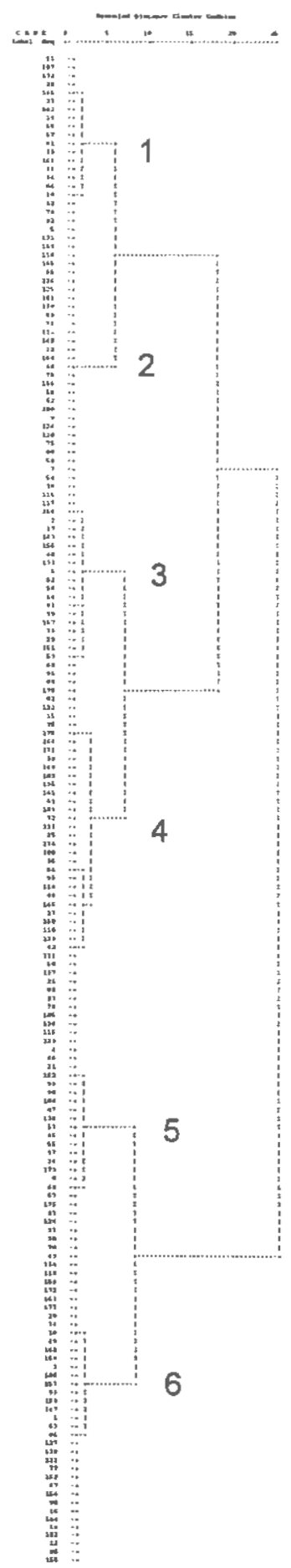

Figure 2. Dendrograms from the cluster analysis of the two data subsamples. 

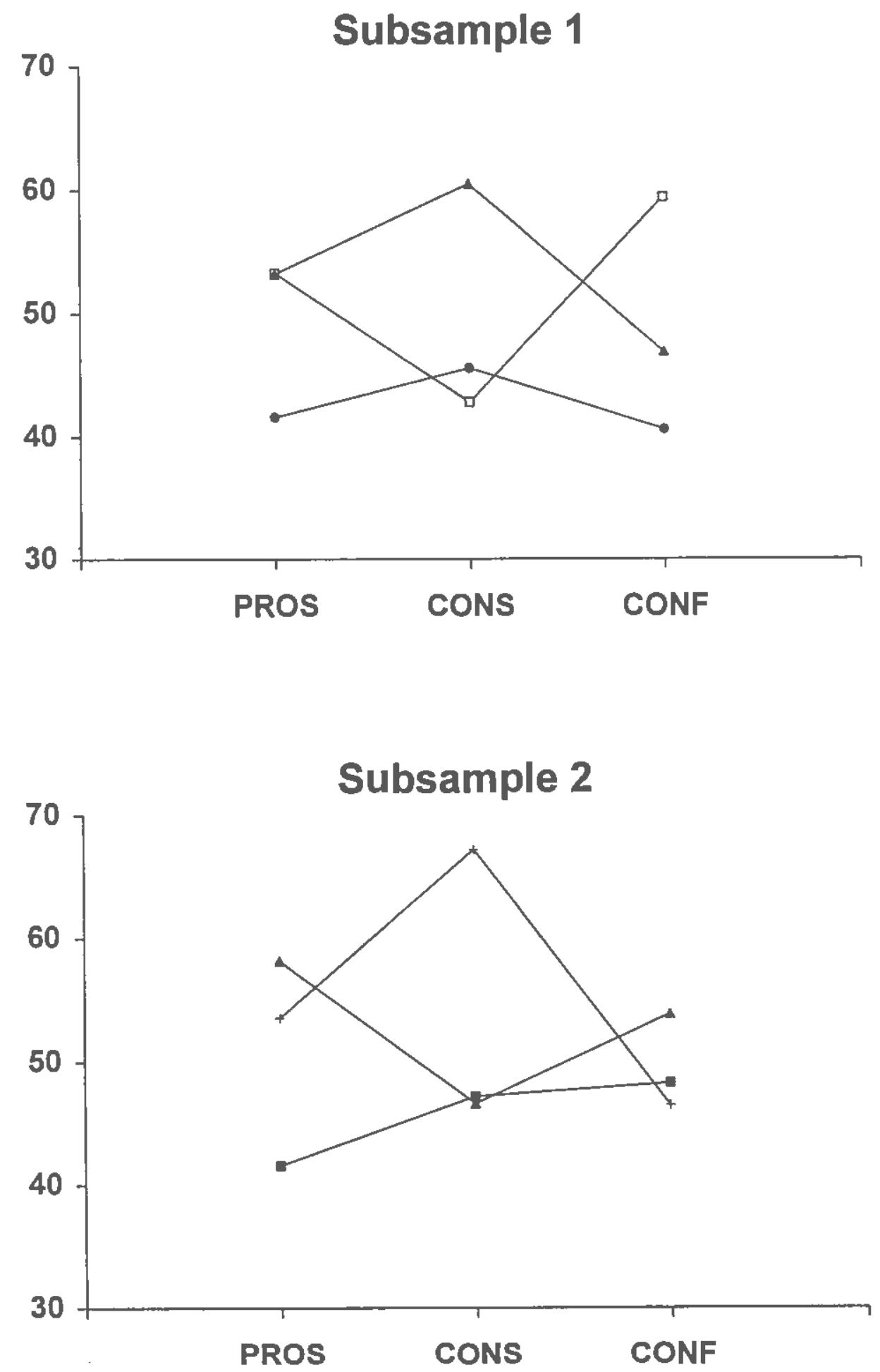

Eigure 3. Profile patterns for the 3 cluster solution. 


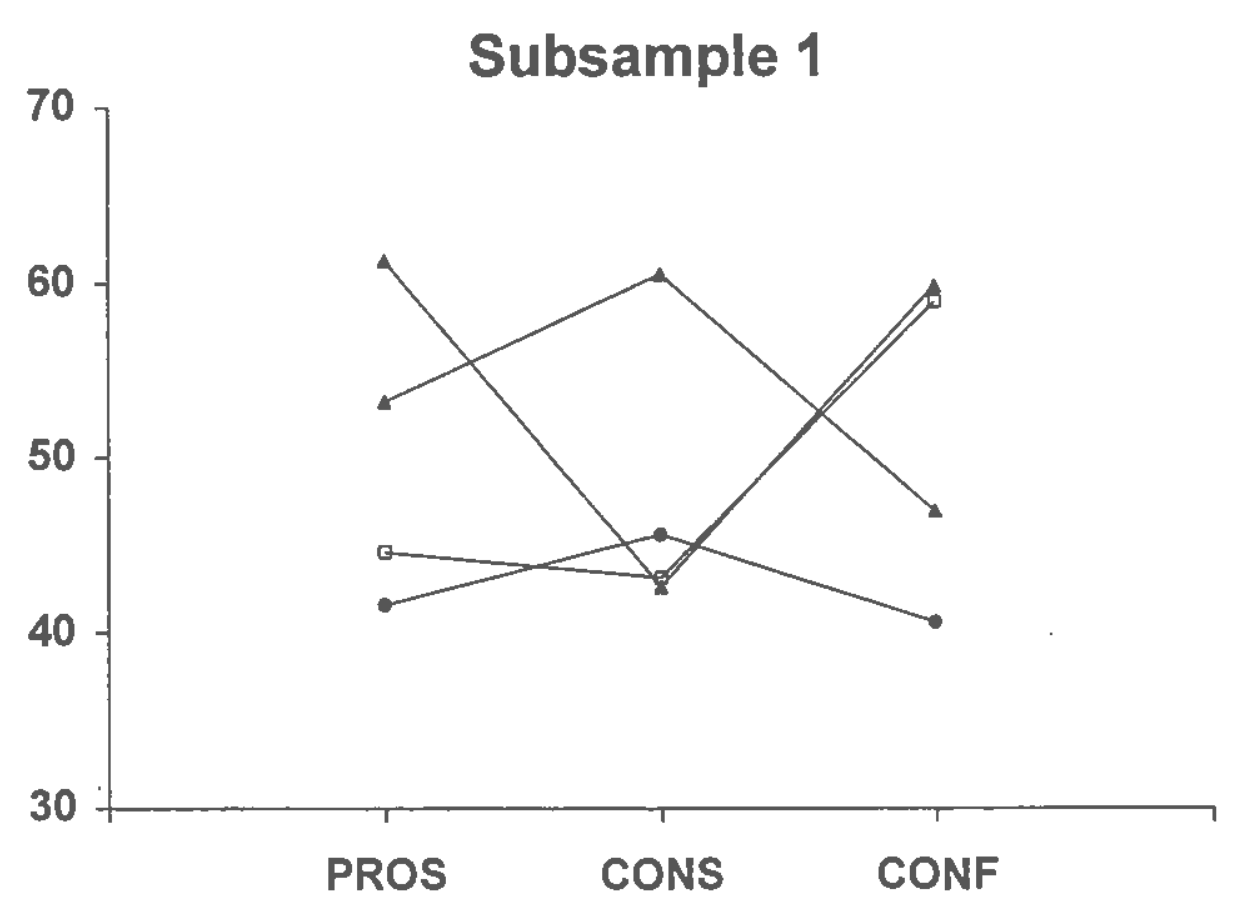

Subsample 1

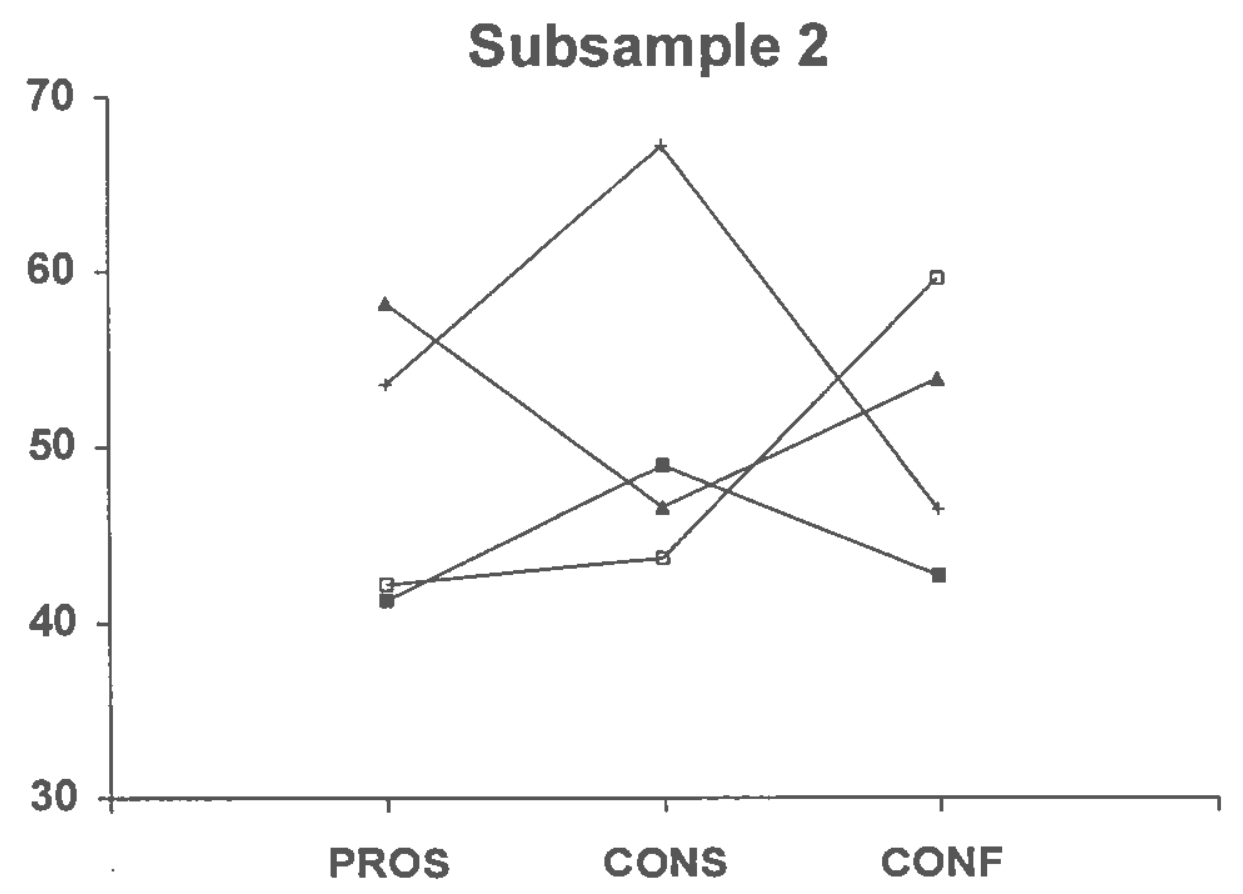

Figure 4. Profile patterns for the 4 cluster solution. 

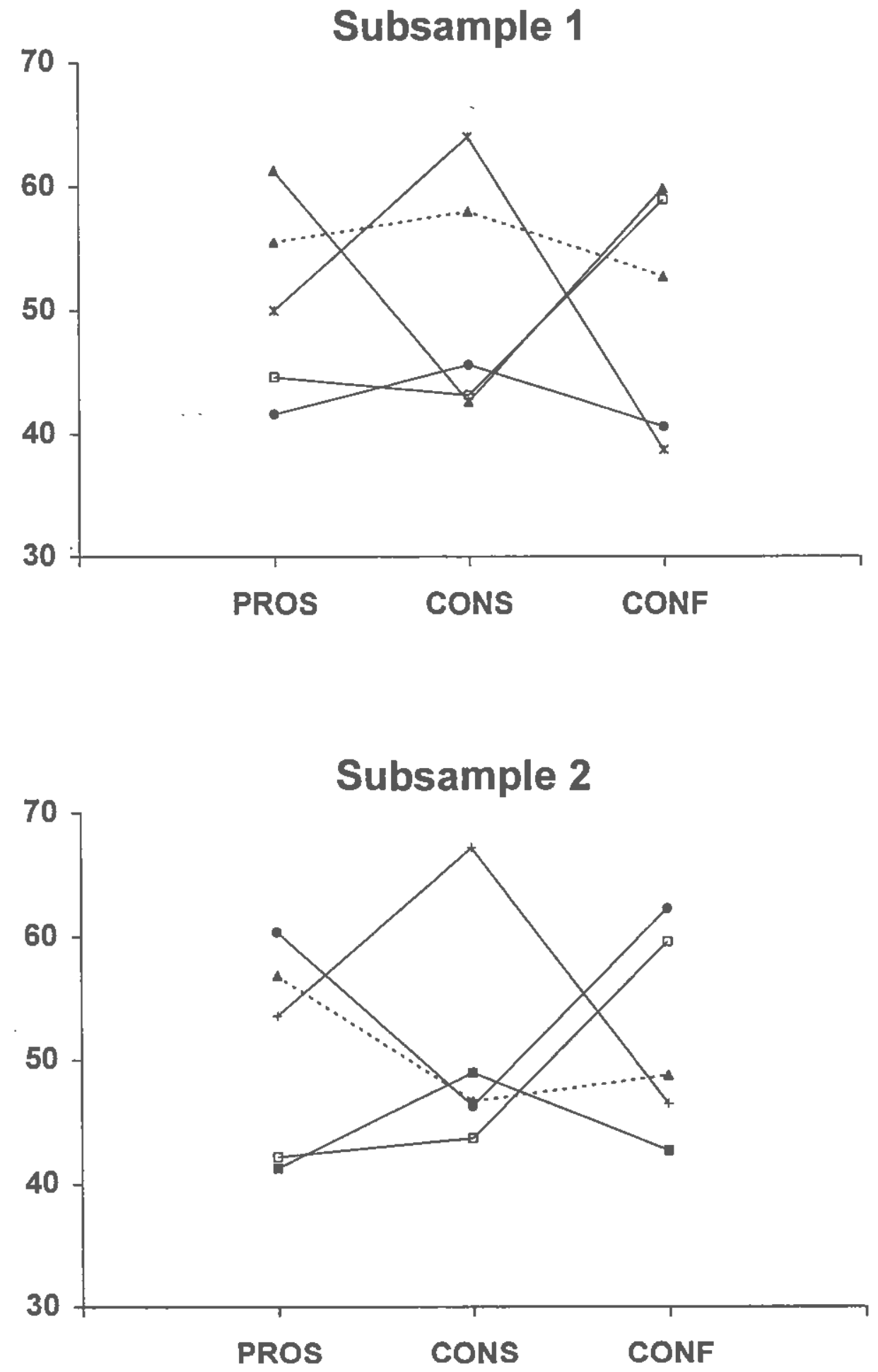

Figure 5. Profile patterns for the 5 cluster solution. 


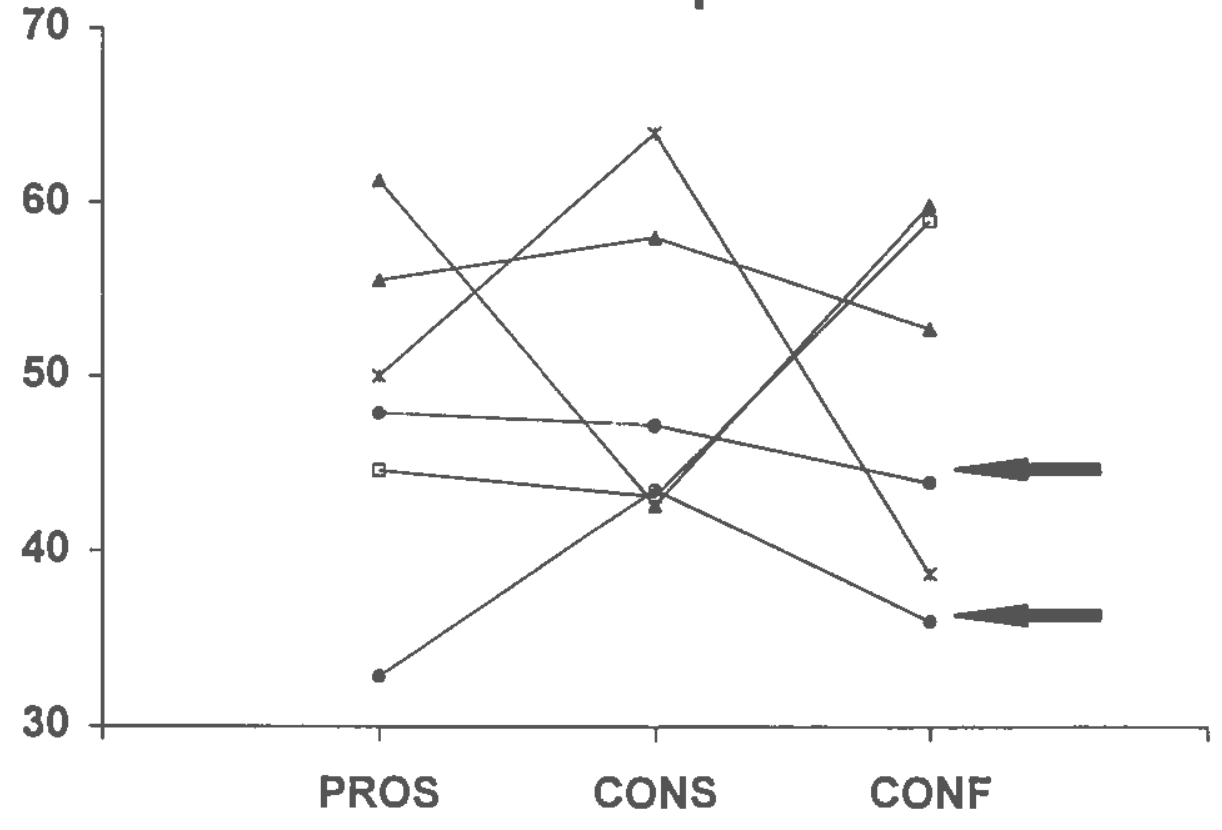

Subsample 1

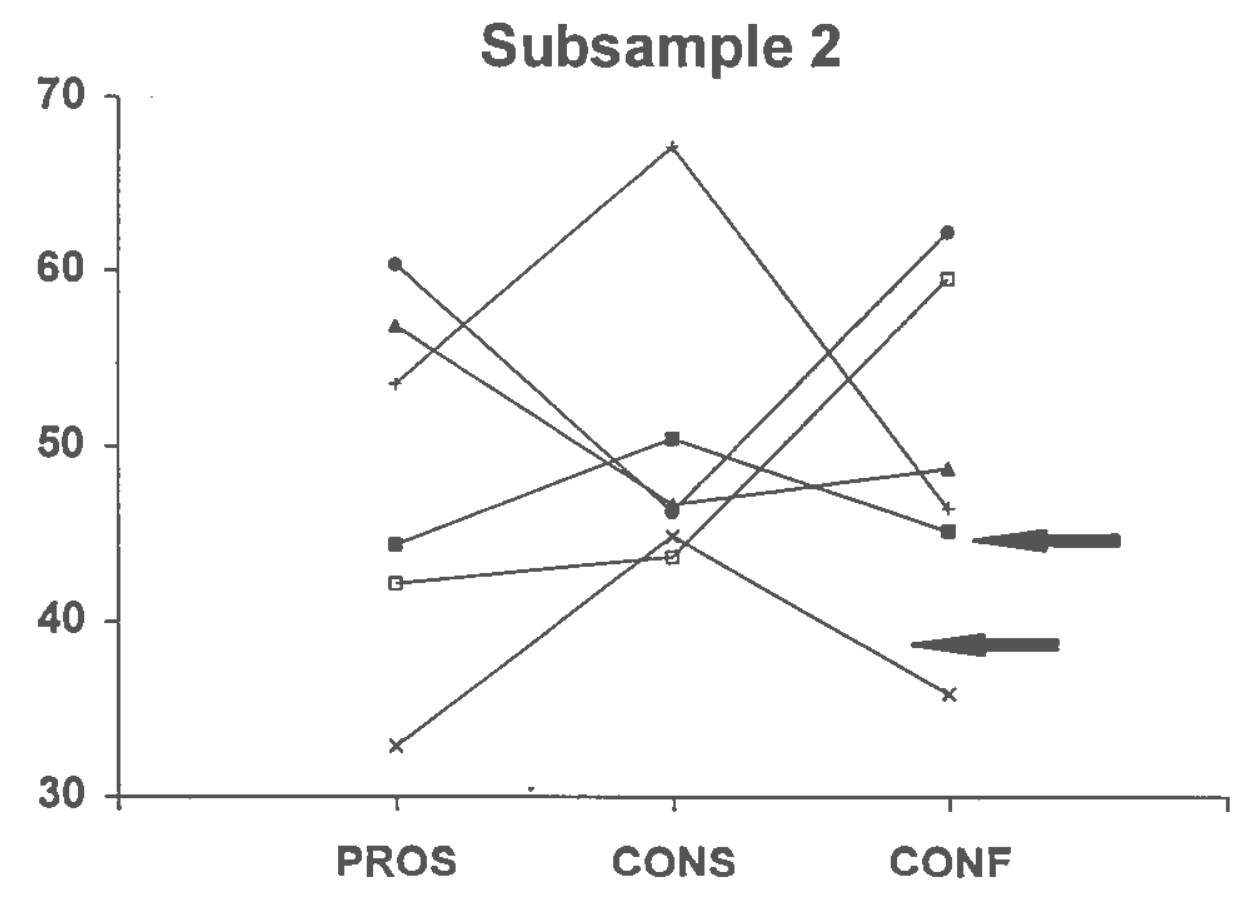

Figure 6. Profile patterns for the 6 cluster solution. 

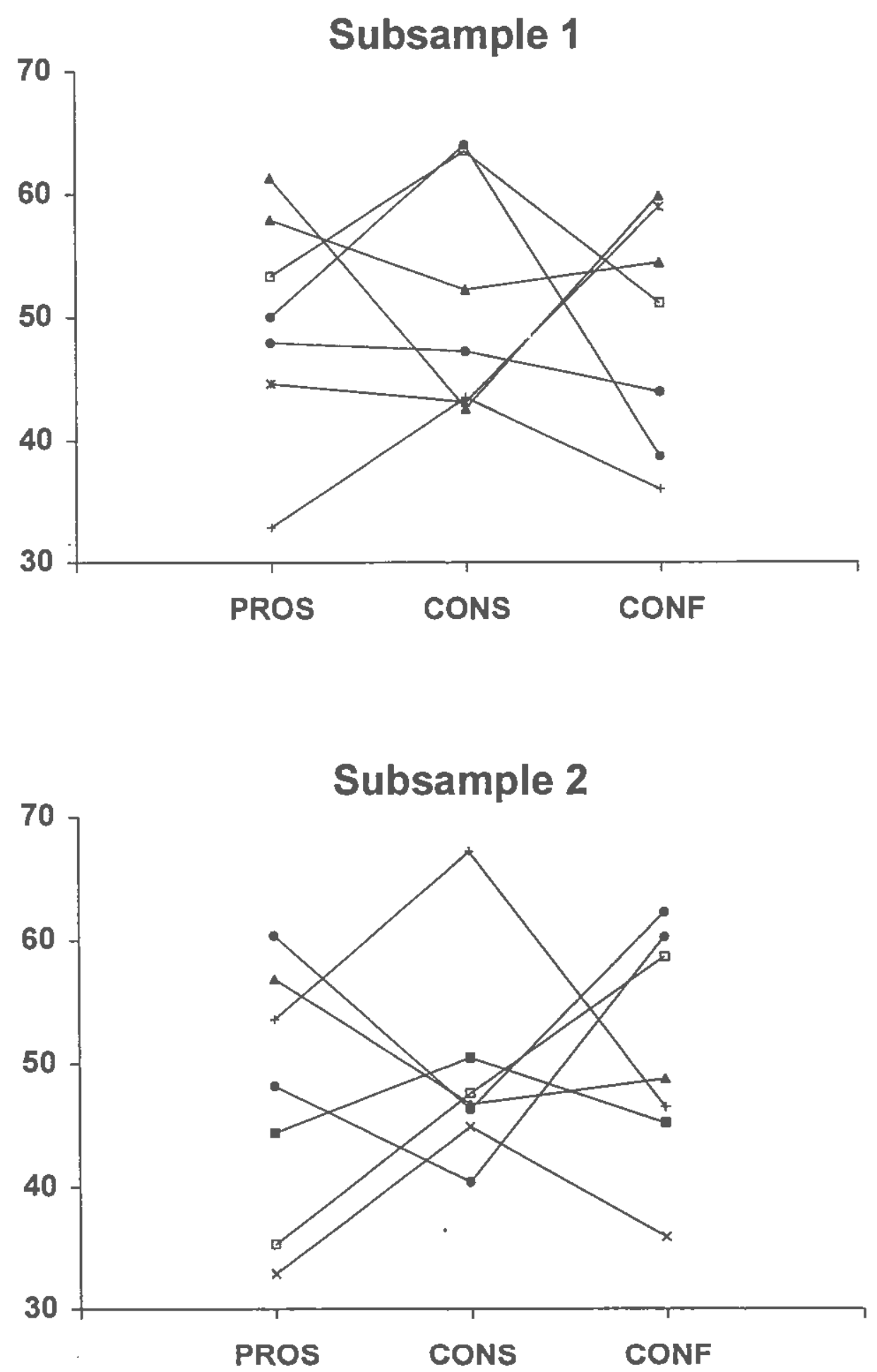

Figure 7 Profile patterns for the 7 cluster solution. 


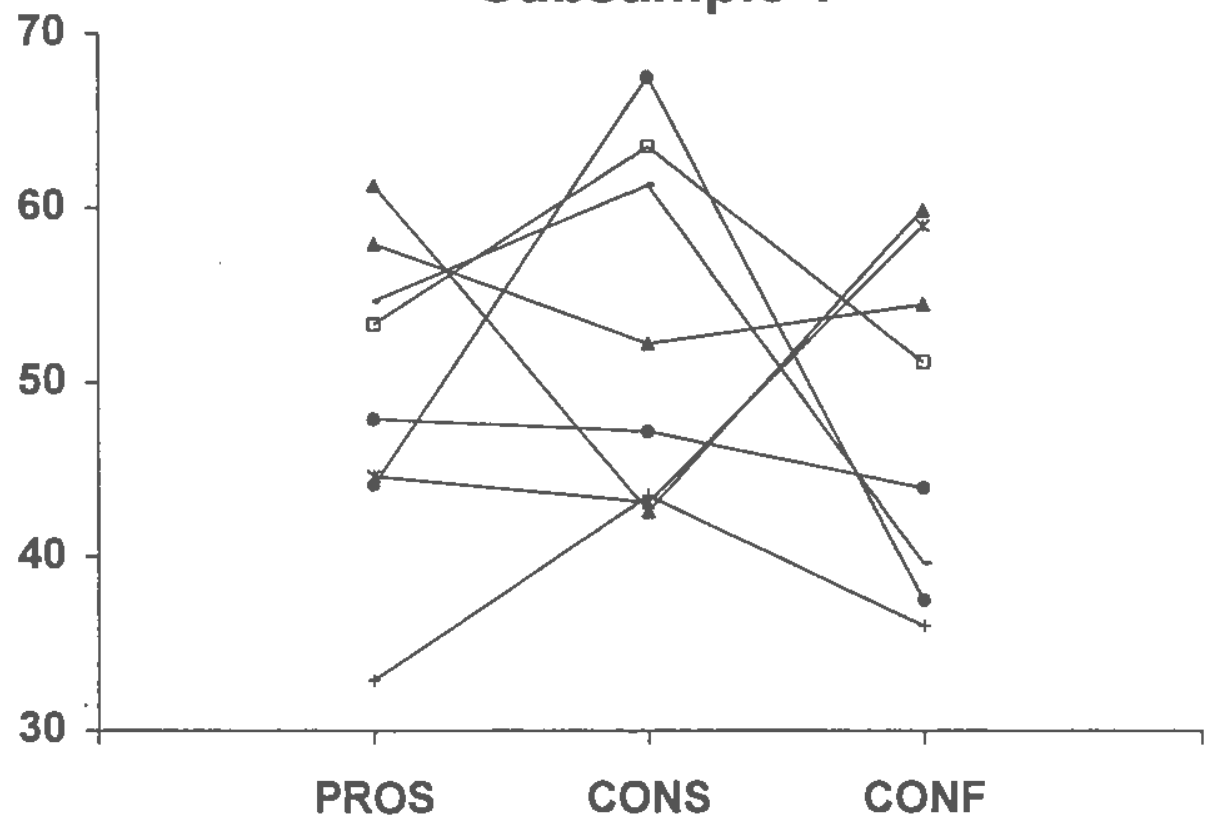

\section{Subsample 2}

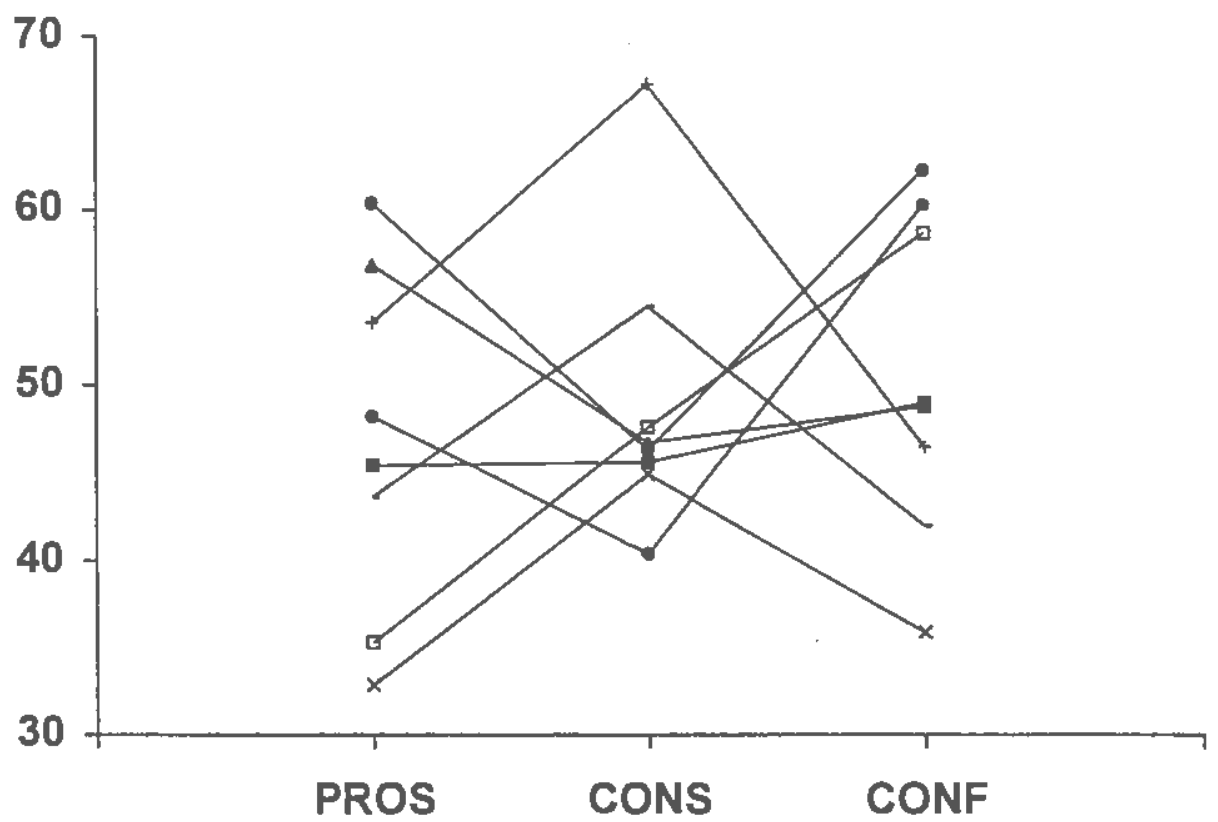

Figure 8. Profile patterns for the 8 cluster solution. 


\section{Describing and Naming the Clusters}

The pattern profiles of the six clusters are presented in Figure 9. Although some of the cluster pattern profiles closely resembled patterns expected of particular stages of change (Velicer et al., 1995), the stage names (e.g. Precontemplation) were intentionally not given to clusters so as to avoid confusion when comparing stage of change groups to cluster groups.

Cluster 1. The profile of Cluster 1 had a flat shape, low elevation, and little scatter. This cluster was named Disengaged because the Pros, Cons, and Confidence were all at the same level, between one half and one standard deviation below the mean. These individuals did not see the pros or the cons as very important and their confidence to exercise regularly was low. Disengaged profiles have also been found for smoking behavior in previous research and represent a category of individuals that were not originally hypothesized within the transtheoretical model (Velicer et al.; 1995, Norman et al. 1997).

Cluster 2. Individuals in Cluster 2 had a pattern profile that was very characteristic of early stage individuals. Consequently, this cluster was named Early. The shape of the pattern is a very distinct inverted ' $\mathrm{V}$ ' with average level and high scatter. The Pros were about average, the Cons were about one and a half standard deviations above average and Confidence was almost a full standard deviation below average. For smoking behavior, this type of pattern has been called Immotive and represents the pattern expected of precontemplators (Velicer et al., 1995). 
Cluster 3. The mean profile for this cluster was characterized as having a flat shape, a high elevation, and very little scatter. The Pros, Cons, and Confidence were all about a half standard deviation above average. This group seemed to be at a critical point in relation to exercise. The Pros and Cons were equally elevated indicating conflict between weighing out the positive and negative aspects of exercising. Confidence to exercise regularly was above average but not at the level of some of the other clusters. This cluster was named At Risk because although individuals in this cluster may have been active, their profile is characteristic of individuals in the Preparation stage.

Cluster 4. The profile shape of Cluster 4 resembled an ' $L$ ' with average level and moderate scatter. The Pros were almost a standard deviation above average while the Cons and Confidence were about average. This profile was characteristic of individuals in either the Preparation or the Action stage. The Pros outweighed the Cons but Confidence was at an average level. This profile was named Early Action.

Cluster 5. Cluster 5 was the classic pattern expected of individuals who have progressed through the stages of change and have been exercising regularly for some time. This cluster was named Maintainers. The shape of the cluster was a distinct ' $\mathrm{V}$ ' with average level and high scatter. The Pros were about a standard deviation above the mean, the Cons were almost a standard deviation below the mean, and Confidence was about a standard deviation above the mean. For these people the benefits of exercise were important and they were confident that they could exercise regularly.

Cluster 6. This cluster was characterized as having the Pros and Cons about a half standard deviation below average and Confidence about a standard deviation above 
average. The shape of this profile resembled a backwards ' $L$ '. This group was labeled Habituated because the profile depicted exercisers who were very confident in their ability to exercise regularly yet did not find either the Pros or Cons of exercising as important in their decision to exercise regularly. It was hypothesized that to these individuals exercise was probably a well formed habit. 


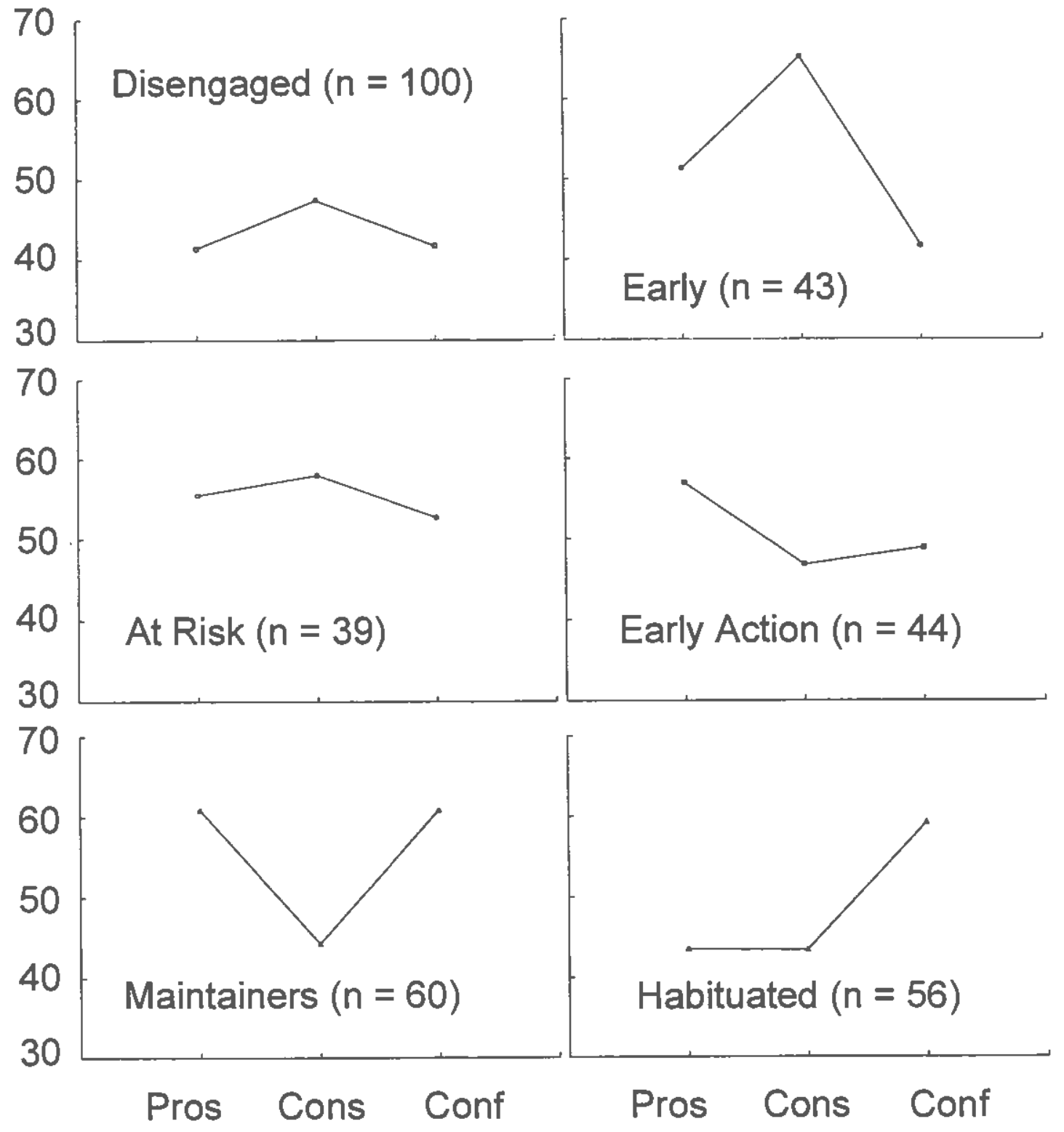

Figure 9. Six cluster profiles based on Pros, Cons, and Confidence. 
Internal Validation: Random Subsample Clustering

Using replication as a step in cluster analysis has been recommended as a means of validating cluster solutions (Overall \& Magee, 1992; Breckenridge, 1989). This can be considered a type of internal validity since the same variables used to perform the initial cluster analysis are being used to validate the analysis (Milligan, 1981b). To examine the consistency of recovering the cluster patterns, ten random samples of size $n=170$ were consecutively drawn from the total sample $(n=342)$.

Cluster analysis was performed using Ward's method and squared Euclidean distance on each of the 10 random samples. Cluster profiles were then plotted for cluster solutions four through six. The object was to identify as many of the previously found six cluster profile patterns in each of the random sample plots. A high percentage of cluster profile pattern replication was considered an indicator of reliable cluster. If the random subsamples produced different or inconsistent patterns this would indicate that clusters were not reliable and that the data did not contain clearly defined groups.

A cluster profile pattern had to be clearly evident for it to be labeled one of the six patterns of Early, Maintainers, Disengaged, Habituated, At Risk, or Early Action. Quantifiable detection rules were established for each pattern based on profile shape, level, and scatter. For example, the At Risk profile which was characterized as having a flat shape, low scatter, and moderate level could only be identified if it was between 50 and $60 \mathrm{~T}$-scores and all variables (pros, cons, confidence) were within a half standard deviation (5 points) of each other. 
Table 12 summarizes the findings from this analysis. First, two patterns, Maintainers and Disengaged, were consistently detected in cluster solutions 4,5 , and 6 for all 10 random samples. Second, the Early and Habituated patterns were nearly always detected. The high scatter expected of the Early pattern was reduced somewhat by the 5 and 4 solutions. Third, the last two patterns were less consistent. The At Risk and the Early Action patterns were the two patterns that were not replicated in the five cluster solution of the original cluster analysis. They were clearly present in about half the samples for the six cluster solutions but commonly merge with other patterns in the 5 and 4 cluster solutions.

Out of the 150 sets of cluster pattem profiles ( 10 random samples $x(6+5+4$ cluster solutions)) 14 patterns did not match one of the 6 cluster patterns. Overall, four of the patterns were consistently reproduced, while two patterns were found inconsistently, and the majority of the patterns were one of the six target patterns. These results were interpreted as evidence for reliable and replicable clusters. 
Table 12

Summary of Cluster Pattern Replication in Ten Random Subsamples

Cluster Solution

Cluster Profile

6

5

4

Early

Present in 10

samples. High

scatter with pros $\approx$

50 , cons $>60$, conf $\approx 40$.

Present in all 10 samples.

Present in all 10 samples. For some samples $(1,2,3,8)$ shape more $\Lambda$ with low level, moderate scatter.
Present in 8

samples. 2 remaining samples,

$\Lambda$ shape but moderate scatter $(2,3)$

Present in all 10 samples.

Present in all 10 samples.
Present in 7

samples. 3

remaining samples,

$\Lambda$ shape but moderate scatter $(2,3,8)$.

Present in all 10 samples.

Present in all 10 samples.

Habituated

Present in 9 samples

Present in 9 samples

Present in 7 samples (not found in sample 2).

(not found in sample 2). (not found in 1,5,9). Emerges in sample 2.

At Risk Present in 6 samples $(1,3,5,7,8,10)$. Present in 4 samples $(1,3,5,8)$.

Present in 2 samples $(1,5)$.

Early Action

Present in 4 samples Present in 3 samples $(1,4,5,6,9)$. $(4,6,9)$.

Present in 1 sample (9).

Note. Size of all random subsamples was $n=170$. 


\section{Internal Validation: Double Cross-Validation}

A cross-validation procedure was used as a test of the stability, or replicability, of the cluster solution. Assessing the stability of a cluster solution is similar to assessing the reliability of a psychological test (McIntyre \& Blashfield, 1980). Just as reliability is a necessary but not a sufficient condition for establishing a test's validity, the same is true of cluster stability. Since the "true" classification of individuals in a data set is not known, there is no direct measure of the accuracy of a cluster solution. Mclntyre and Blashfield (1980) found in a simulation study that the accuracy of a solution could be estimated by assessing its stability. Specifically, they found that when using the nearestcentroid procedure to evaluate minimum variance cluster solutions, the correlation between stability and accuracy of the cluster solution was as high as .97 . This magnitude of association was found when the cluster variables had low inter-correlations and were normally distributed.

For the present study the same cross-validation sequence was used as described by Mclntyre and Blashfield (1980). The stability of the 5 cluster solution was assessed for Subsample $1(n=177)$ and Subsample $2(n=165)$, which were clustered using Ward's minimum variance method (Ward, 1963). The steps for the cross-validation procedure were as follows:

1. Subsample 1 was cluster analyzed.

2. The centroid vectors for each cluster from the 5 cluster solution were calculated.

3. Subsample 2 was cluster analyzed. 
4. The squared Euclidean distance for each of Subsample 2's individuals from each of the centroids of Subsample 1 was calculated.

5. Each individual in Subsample 2 was assigned to the closest centroid vector.

6. Agreement between the nearest-centroid assignment of step 5 and the cluster results of step 3 were measured with the kappa statistic (Cohen, 1960). Kappa is a measure of agreement that is adjusted for agreement accounted for by chance. It can range from 1.0 (perfect agreement) to 0.0 (no agreement).

7. The same series of steps was conducted reversing the roles of Subsample 1 and Subsample 2 for double cross-validation of the 5 cluster solution.

Table 13 presents the cross-validation of Subsample 2 replicating Subsample 1. Agreement between the cluster analysis and the nearest-centroid classification was $66.7 \%$. The kappa statistic was $.59(\mathrm{t}=16.17, \mathrm{p}<.001)$ indicating a high degree of replication. Similar results were found for the cross-validation of Subsample 1 replicating Subsample 2 (See Table 14). Here the overall agreement was $72 \%$ with a statistically significant kappa of $.65(t=17.48, p<.001)$. These indices of agreement exceed levels of agreement found in two other studies where this procedure was used in applied research settings. Morey et al. (1983) found an average adjusted Rand statistic score of .345 across 23 clustering procedures. Humphreys and Rosenheck (1995) reported an adjusted Rand score of .463 , which was the largest score from 12 clustering methods. The Rand and the kappa indices are highly correlated and are essentially identical for practical applications (Milligan, 1981b). Based on the results of Morey et al. (1983) and Humphreys and Rosenheck (1995), measures of agreement adjusted for 
chance that exceed .500 are an indication of good cluster stability. If that heuristic is correct, then the cluster solution in the present study demonstrated high stability. It is also of note that in the present study, when a subsample was cross-validated with itself (e.g. assignment of Subsample 1 using centroid vectors of Subsample 1), kappa values for Subsample 1 and Subsample 2 were .85 and .87 respectively.

The largest discrepancy was found for cluster 3 which was the cluster pattem that differed between the two subsamples. In Subsample 1 this cluster was labeled At Risk and in Subsample 2 the cluster was labeled Early Action. The pattern of discrepancies is evidence of a qualitative difference between the At Risk and Early Action clusters. For the At Risk cluster in Subsample 1, most of the missclassified individuals were assigned to the preceding cluster, which was labeled Early. On the other hand, in Subsample 2, most of the misclassified individuals in the Early Action cluster were assigned to the succeeding cluster, which was labeled Maintainers. These discrepancies were really not misclassifications, but rather clusters that did not have a corresponding cluster to which to be assigned. The results of the cross-validation analysis corroborate the findings from the random subsample clustering test. Four of the clusters demonstrated high stability (Disengaged, Early, Maintainers, Habituated), while two patterns were less stable as a function of being unique to a subsample. 
Table 13

Cross-Validation of Subsample $2(n=165)$ Replicating Subsample $1(n=177)$

Nearest-Centroid Assignment

Cluster

DI ER AR

Analysis:

Subsample 2

$\begin{array}{lllllll} & & & & & & \\ \text { DI } & \text { ER } & \text { AR } & \text { MA } & \text { HA } & \text { Row } \\ & & & & & \text { Total }\end{array}$

DI

35

7

3

7

52

ER

10

6

16

EA

4

2

19

16

3

44

MA

4

23

27

HA

1

2

23

26

Column Total

40

19

32

$41 \quad 33$

165

Note. $\mathrm{DI}=$ Disengaged, $\mathrm{ER}=$ Early, $\mathrm{AR}=$ At Risk, $\mathrm{EA}=$ Early Action, $\mathrm{MA}=$

Maintainer, $\mathrm{HA}=$ Habituated. Kappa statistic $=.59(\mathrm{t}=16.17, \mathrm{p}<.001)$. 
Table 14

Cross-Validation of Subsample $1(n=177)$ Replicating Subsample $2(n=165)$

Nearest-Centroid Assignment

Cluster

DI ER EA MA

HA Row

Analysis:

Total

Subsample 1

DI

39

9

48

ER

$4 \quad 21$

2

27

AR

1

19

15

4

39

MA

6

27

33

HA

1

4

25

30

Column Total

45

40

32

35

25

177

Note. $\mathrm{DI}=$ Disengaged, $\mathrm{ER}=$ Early, $\mathrm{AR}=\mathrm{At}$ Risk, $\mathrm{EA}=$ Early Action, $\mathrm{MA}=$ Maintainer, $\mathrm{HA}=$ Habituated. Kappa statistic $=.65(\mathrm{t}=17.48, \mathrm{p}<.001)$. 


\section{External Validation: Exercise and Physical Activity Behavior}

This is the first of a series of external validation tests of the exercise cluster typology. In each test, statistical significance tests were performed with the clusters serving as the levels of the independent grouping variable to test for differences on relevant dependent measures. Variables used to establish external validation of the cluster solution must be variables that were not used in the cluster analysis but have theoretical relevance to the clustering variables (Aldenderfer \& Blashfield, 1984).

Differences between the exercise clusters on self-reported exercise and physical activity were measured with two assessments. The first was the Godin Leisure Time Exercise Questionnaire (GLTEQ) and the second was the Paffenbarger/Havard Alumni Questionnaire (P/HAQ) (see Appendix). Separate ANOVAs were conducted with cluster membership as the independent variable. Tukey HSD post hoc tests were computed to determine statistically significant mean differences.

Exercise Behavior. The GLTEQ assesses the number of times individuals participate in leisure time strenuous, moderate, and mild exercise activities. A weighted summed score was calculated to create a composite score of estimated sum of METS per week. The formula for this total score was $((9 x$ strenuous $)+(5 x$ moderate $)+(3 x$ mild)). The weights represented average METS for the different activity levels (Godin \& Shepard, 1985).

Table 15 presents the results from the four ANOVAs. A significant difference was found for the weighted total score $(p<.001)$ with a large effect size of .11 . The Tukey pattern of means indicated that the Disengaged and Early clusters engaged in less 
leisure time exercise than the Habituated, Early Action, and Maintainer clusters (see Figure 10). Examining the individual scales revealed that this difference was a function of the amount of strenuous and moderate exercise $(p<.001)$ (see Figure 11). The amount of mild exercise did not differ across clusters.

Physical Activity. The Paffenbarger/Harvard Alumni Questionnaire (P/HAQ) assessed the number of hours a day individuals engage in five different types of activity (vigorous, moderate, light, sitting, and sleeping or reclining). Examples of each type of activity were presented in the assessment (see Table 5). Participants were asked to estimate the number of hours they engaged in each type of activity separately for a typical week day and a typical weekend day. The number of hours that an individual reported for the five types of activities should sum to a total of twenty-four hours.

Separate ANOVAs were conducted for each type of activity for weekday and weekend day for a total of 10 ANOVAs. A Bonferroni correction to the alpha level to control for Type I errors set the alpha level at .005 . None of the ten tests were statistically significant at this alpha level. However, weekday vigorous activity and sitting activity were statistically significant at $\mathrm{p}<.05\left(\mathrm{~F}(5,336)=2.80, \eta^{2}=.04\right.$, and $F$ $(5,336)=2.37, \eta^{2}=.04$ respectively). Tukey post hoc follow-up tests indicated that the Habituated cluster engaged in more hours of weekday vigorous activity $(\underline{m}=2.25)$ than did the Disengaged cluster $(\underline{m}=1.21)$, and the Early cluster engaged in more hours of weekday sitting activity $(\underline{m}=4.98)$ than did the Maintainer cluster $(\underline{m}=3.63)$.

The results from the GLTEQ and the P/HAQ indicated that differences in activity behavior among the six clusters is specific to strenuous and moderate volitional exercise 
behavior. The clusters were not related to types of non-exercise daily activity as indicated from the $\mathrm{P} / \mathrm{HAQ}$ results. 


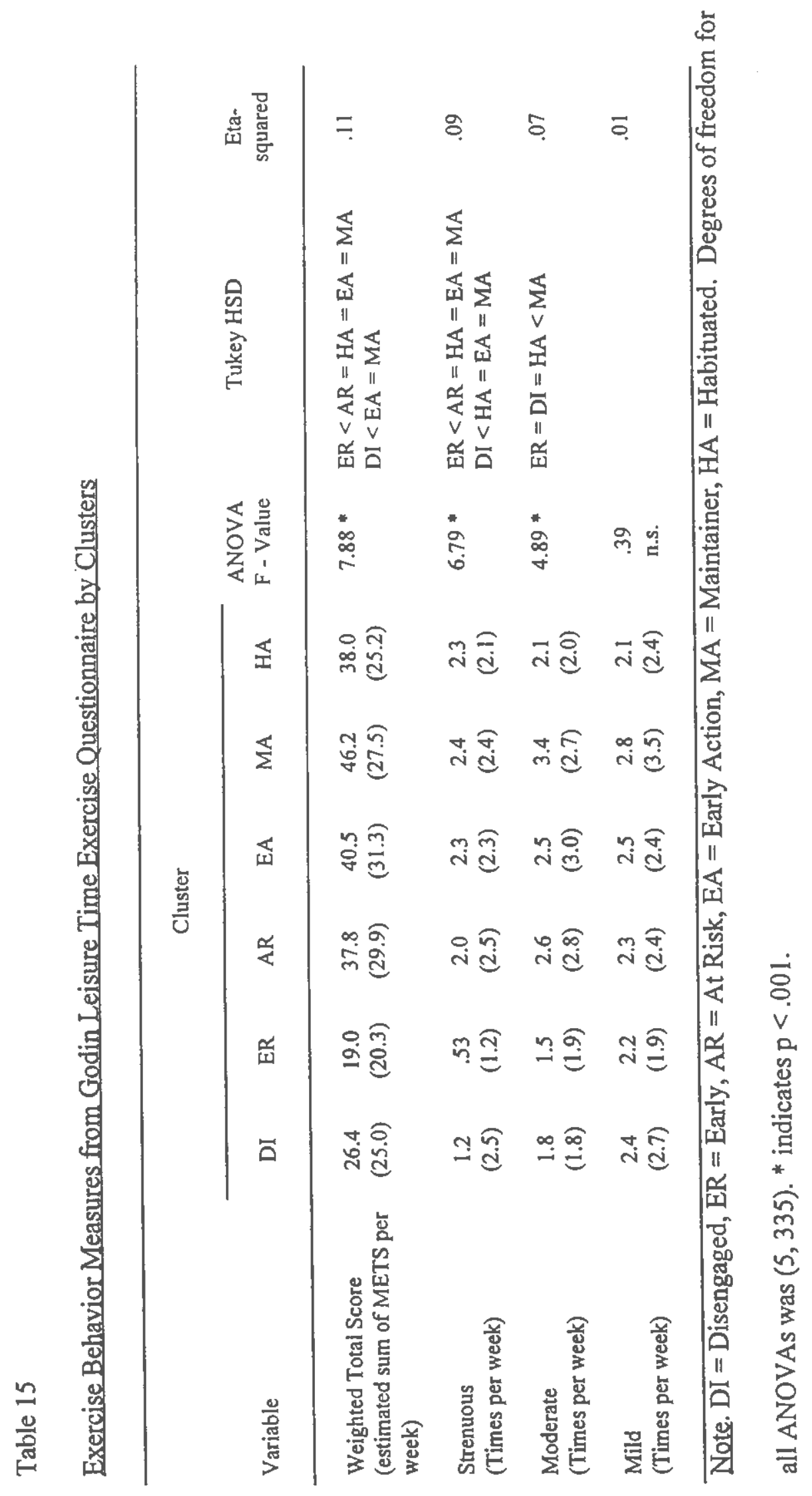




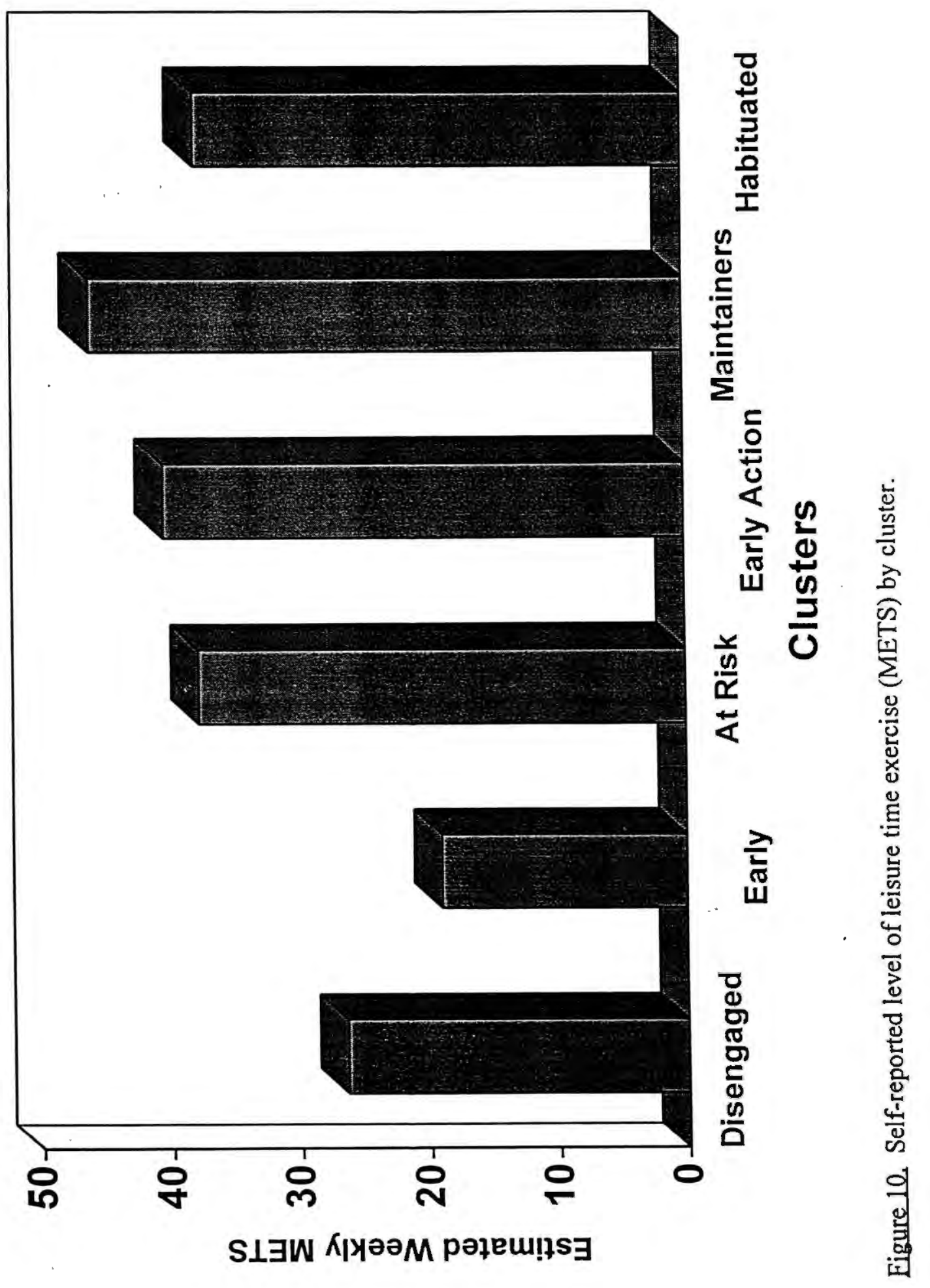




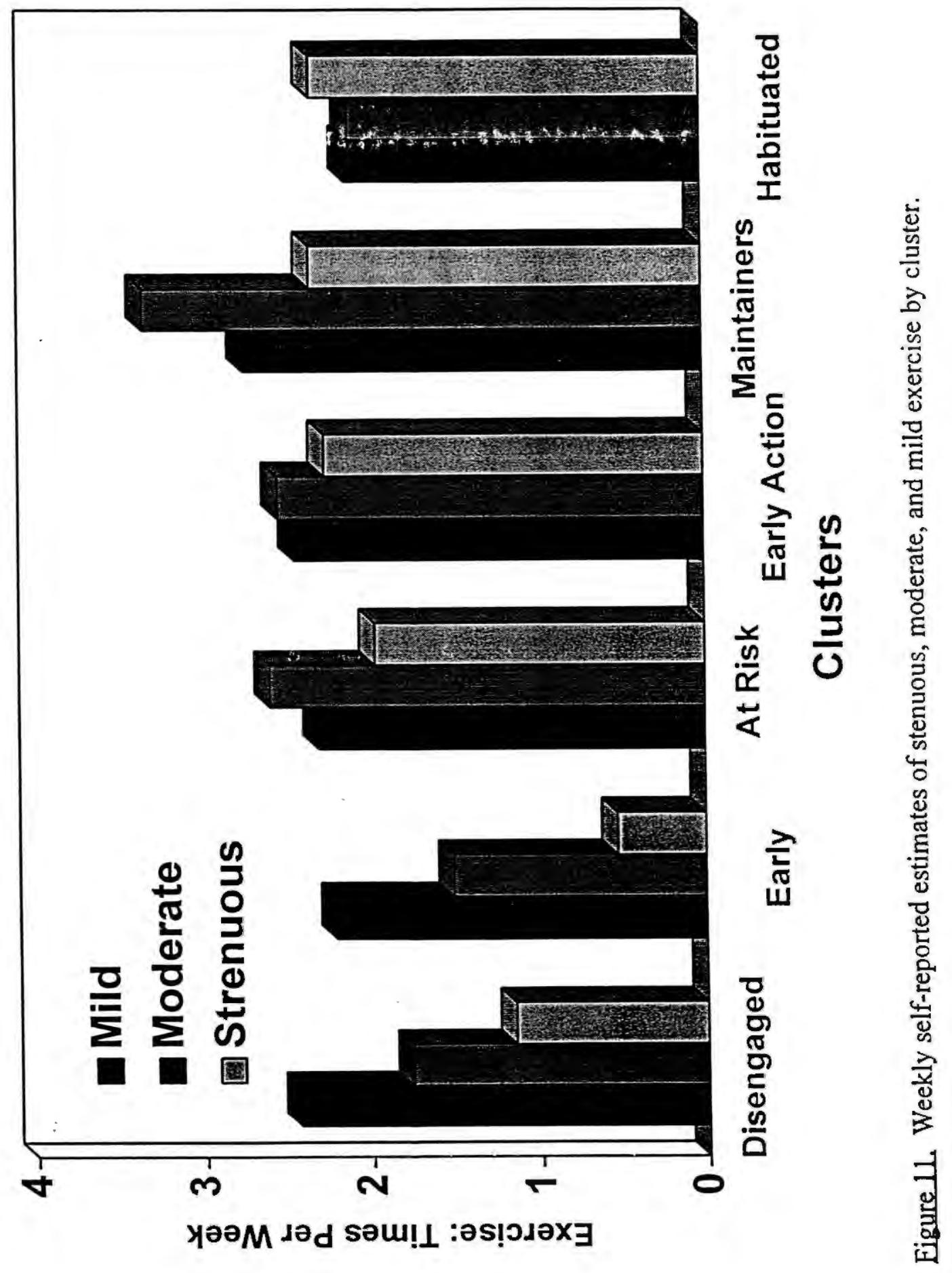




\section{External Validation: Processes of Change}

The ten exercise processes of change were used as dependent external validating variables in two multivariate analyses of variance (MANOVA). The first analysis was on the five experiential processes: Consciousness Raising, Dramatic Relief, Environmental Reevaluation, Social Liberation, and Self Reevaluation. The second analysis was on the five behavioral processes: Reinforcement Management, Helping Relationships, Counter Conditioning, Self Liberation, and Stimulus Control. Cluster membership served as the levels of the independent variable. Statistically significant multivariate tests were followed up with univariate analysis of variance (ANOVA) and Tukey HSD tests. Eta squared effect size measures were calculated and the magnitude of effect sizes were classified as either small (.01), medium (.06), or large (.14) as recommended by Cohen (1992) for social science research.

Experiential Processes. A statistically significant multivariate effect was found for the 5 experiential processes of change (Wilk's $\Lambda=.6005, F(25,1,234)=7.27, p<$ .001). Table 16 displays the means, standard deviations, $\mathrm{F}$ values, Tukey pattern of differences, and measures of effect size for the experiential processes by cluster groups. Group differences were found for all five of these processes with large effect sizes ranging from .13 to .27 . Experiential process use for the Disengaged, Habituated, and Early groups was consistently below the mean of 50.0, while the At Risk and Early Action groups scored between 52 and 54, and the Maintainer group scored above 57 on all of the processes except Environmental Reevaluation (see Figure 12). The pattern of differences generally indicated that the Disengaged group was using these processes the 
least, while the Maintainers were using them the most. The magnitude of difference between the Maintainer group and the Disengaged, Habituated, and Early groups was at least a full standard deviation for four of the five processes, the exception being Environmental Reevaluation.

Behavioral Proceesses. A statistically significant multivariate effect was found for the 5 behavioral processes of change (Wilk's $\Lambda=.6028, \mathrm{~F}(25,1,234)=7.21, \mathrm{p}<$ $.001)$. Table 17 summarizes the results by cluster groups. Differences were found for all five of the processes with large effect sizes ranging from .14 to .32 . Behavioral process use for the Disengaged and Early groups was consistently well below the mean of 50.0, while the At Risk, Early Action, and Habituated groups scored between 49 and 54, and the Maintainer group scored above 56 on all of the processes (see Figure 13). The pattern of differences indicated that the Maintainer group was clearly using the behavioral processes the most, while the Disengaged and Early groups were using these processes the least. The magnitude of difference between the Maintainer group and the Disengaged, and Early groups was very large, at least a full standard deviation difference on all five of the behavioral processes.

A notable pattern occurred between the use of experiential and behavioral processes related to the Habituated cluster. This group's experiential process use looked more like the two sedentary groups, Disengaged and Early. However, behavioral process use by the Habituated cluster more closely resembled the At Risk and Early Action groups. If the Habituated cluster truly is the most advanced cluster in terms of being a group able to maintain their exercise behavior habitually with little risk of relapse, then 
decreased use of the experiential processes would be expected of this group. Overall, the differences in process use clearly validated the six clusters as distinct groups as indicated by the large magnitude of differences on all ten of the exercise processes of change. 


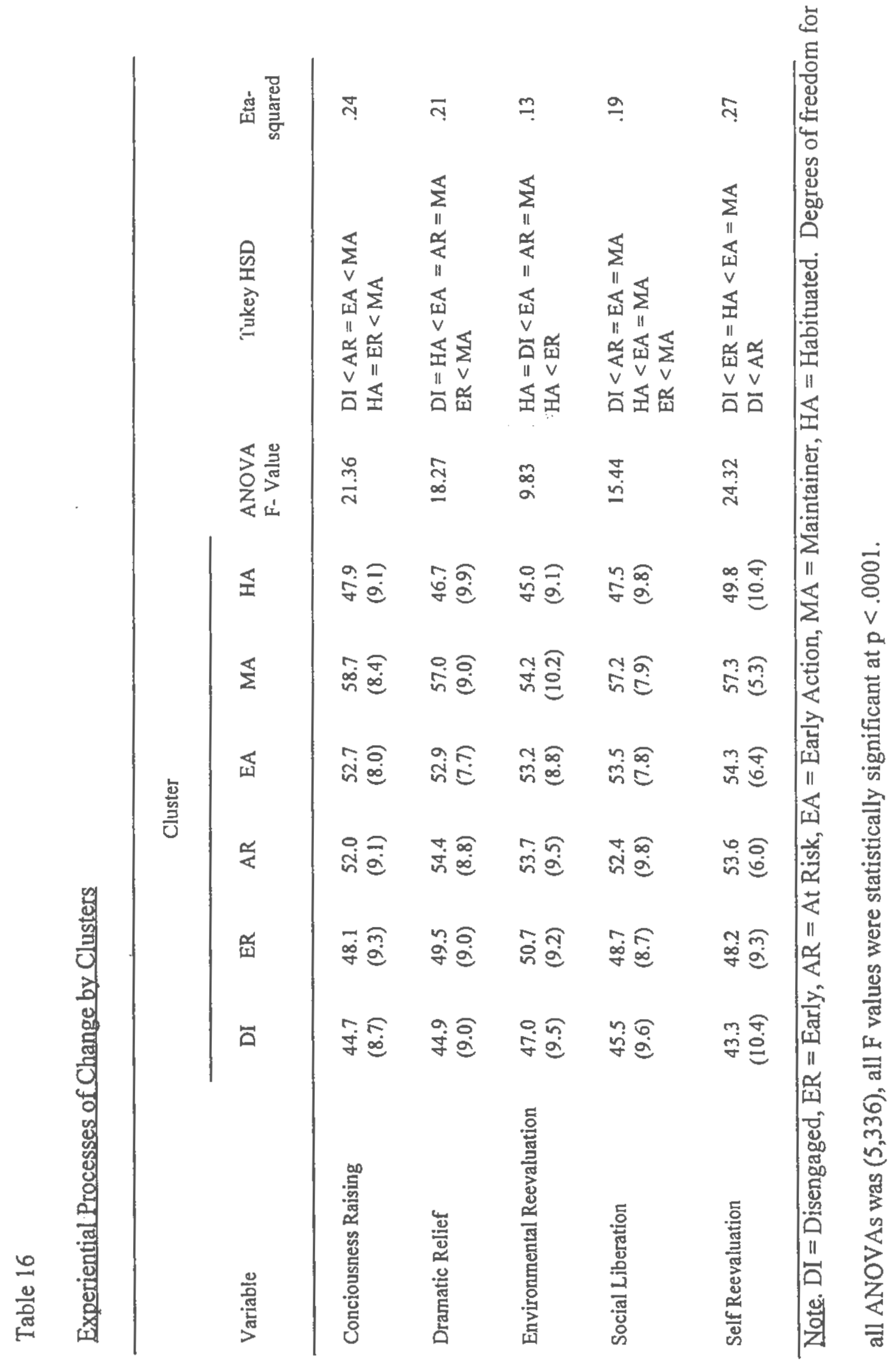




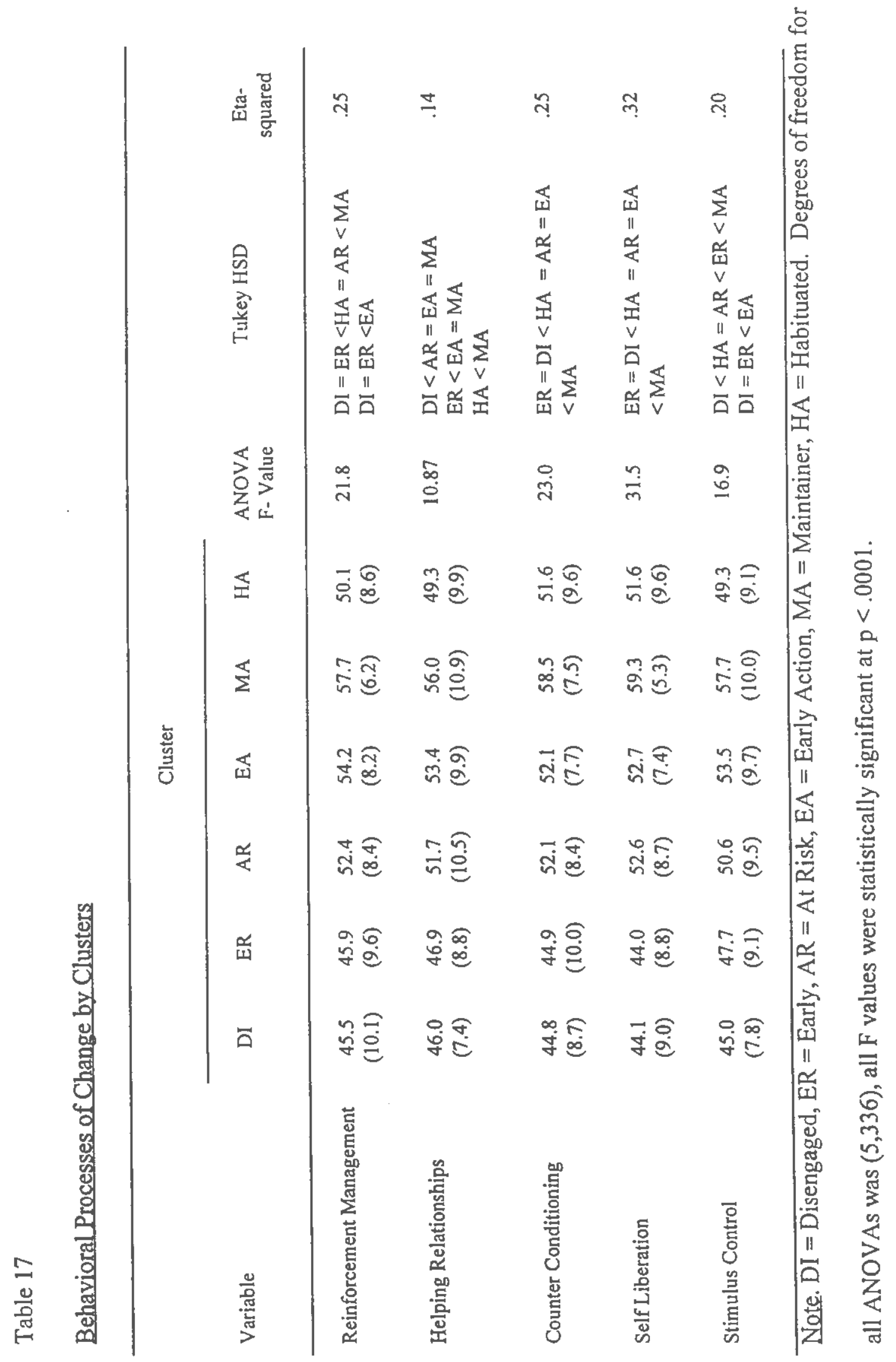




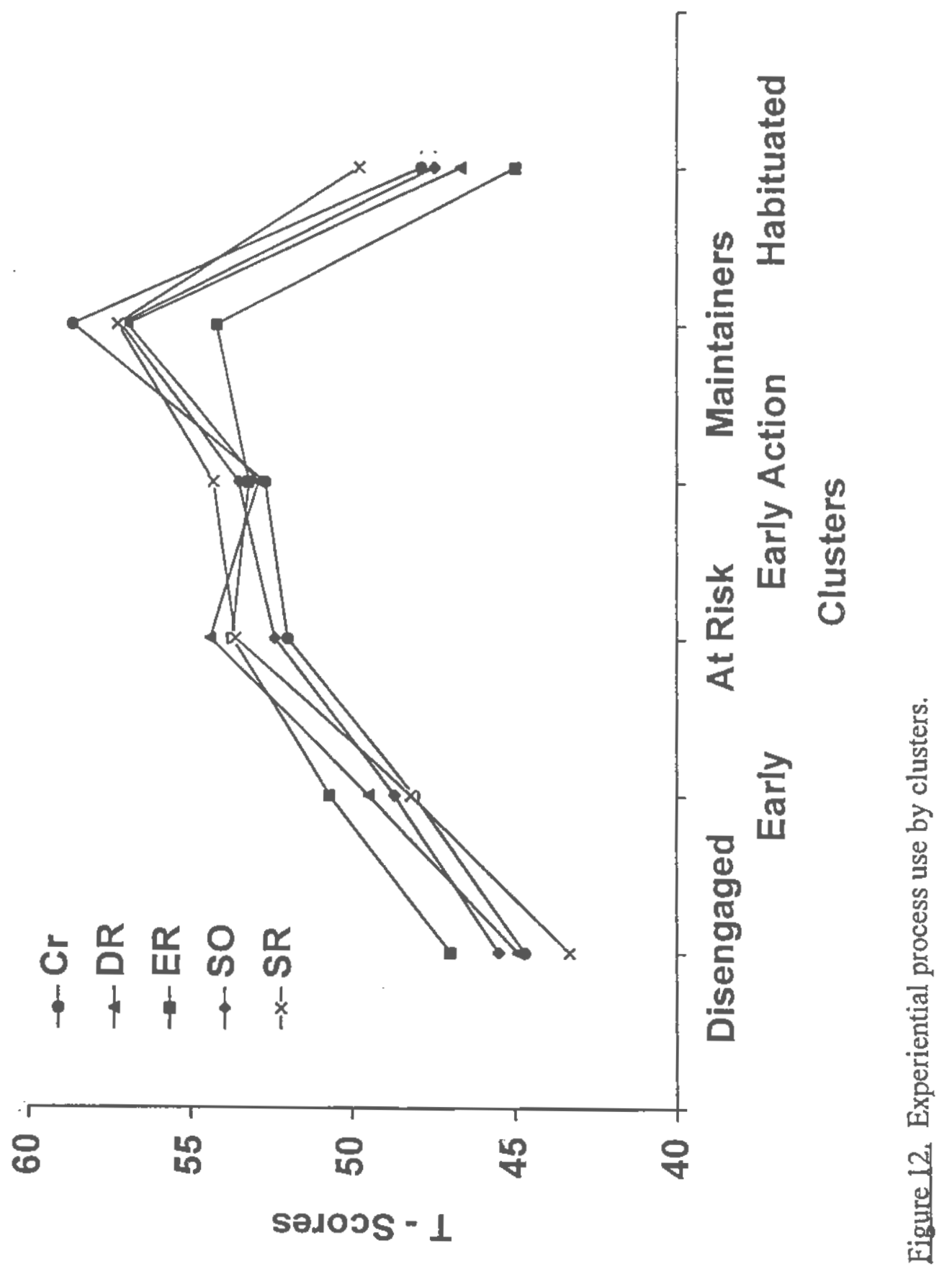




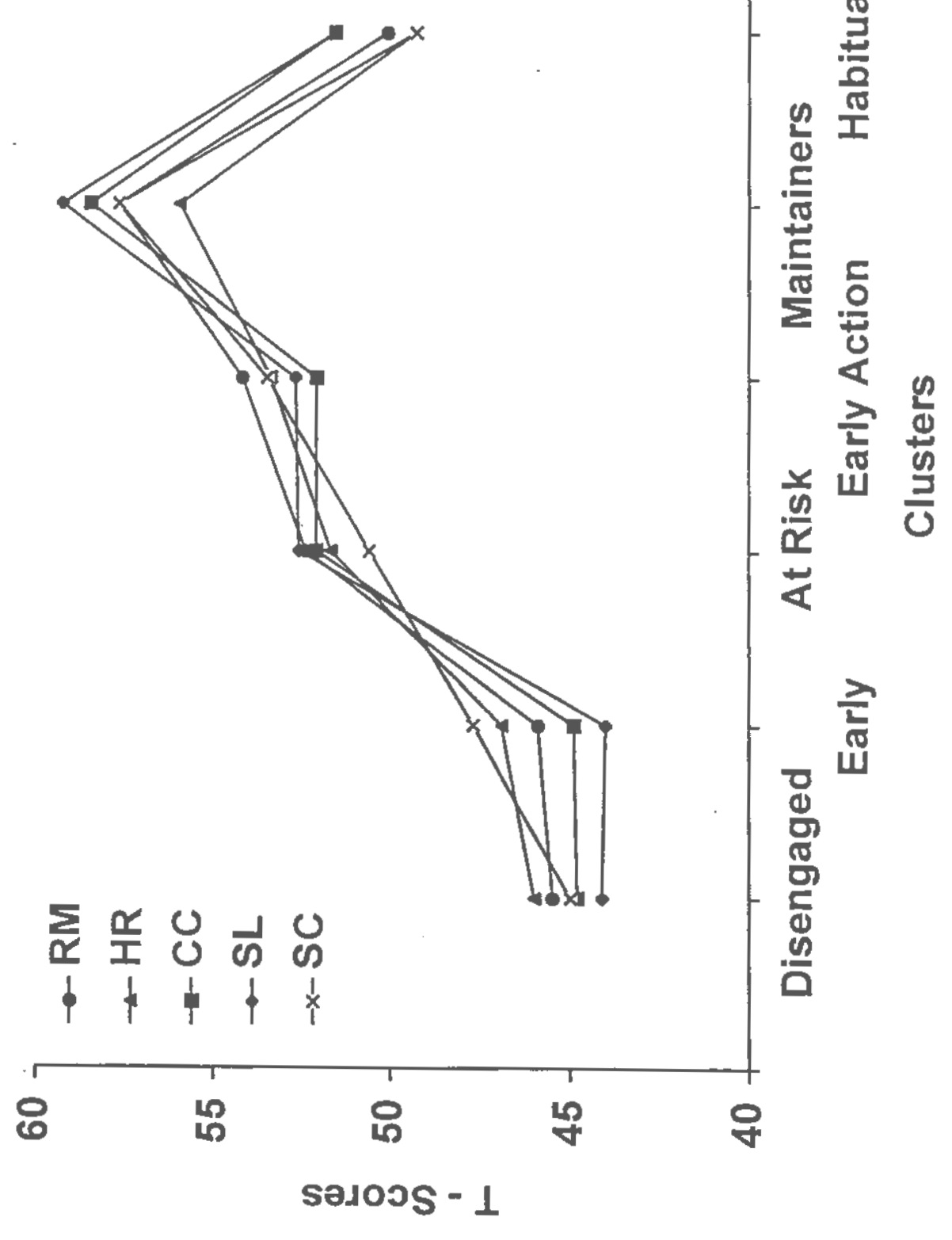

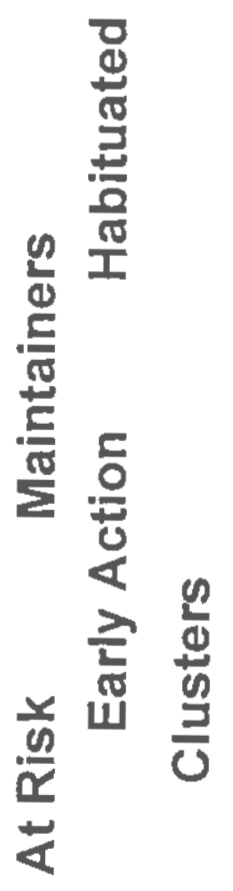

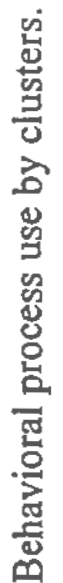

북 


\section{External Validation: Stages of Change for Regular Exercise}

Exercise stage of change was calculated from a four question algorithm. The action criteria was the definition of vigorous exercise recommended by the American College of Sports Medicine (ACSM, 1990), and described the frequency, intensity and duration criteria expected to be considered a regular exerciser. The definition also gave several examples of what is commonly considered regular exercise (see Appendix).

To test the external validity of the six cluster groups a comparison was made with the exercise stages of change. A Chi-square test of association for the $5 \times 6$ contingency table revealed a statistically significant relationship between clusters and stage of change $\left(\chi_{(20)}^{2}=98.8, p<.0001\right)($ see Table 18$)$. To more clearly see the relationship between clusters and stages, the contingency table displayed in Table 18 was recreated in Table 19 to include only cells with clusters that accounted for more than $20 \%$ of a stage's total membership. For example, the cell ${ }_{1.2}$ has a frequency count of 15 , representing the individuals in the Disengaged cluster who were also classified into the Contemplation stage. Thus, the Disengaged cluster accounted for $58 \%$ of the Contemplators in the sample. Each of the remaining clusters accounted for less than $20 \%$ of the Contemplators so these cells were left blank. With one exception, the cells displayed in Table 18 all had frequencies greater than expected by chance and an average standardized residual of 2.3 (ranging from 1.5 to 3.6 ). The only exception to this finding was cell ${ }_{5.4}$ (Maintainer cluster, Action stage) with a standardized residual of .7 , but it was included because the frequency count exceeded the $20 \%$ criteria. 
With the $20 \%$ criteria, the cells which represented strong relationships between the six clusters and the five stages accounted for $58.7 \%$ of the total sample. The remaining cell counts can be attributed to chance. The pattern that emerged between clusters and stages revealed that each stage of change contained a small number of cluster types. The first three stages consisted of the Disengaged and Early clusters. These were the two less active clusters and had profile shapes that would be expected of early stage individuals. The Action stage contained primarily three clusters, the At Risk, Early Action, and Maintainers. The presence of these three cluster groups in the Action stage reflected this stage's volatility where individuals were beginning to exercise regularly at criteria and may progress to Maintenance or relapse back to an earlier stage. The Maintenance stage consisted of primarily the Maintainer and Habituated clusters.

As a further look at the relationship between stages of change and the exercise clusters, the 30 cells from the $5 \times 6$ contingency table were graphed to display the individual pattern profiles which are presented in Figures 14 through 18. Plotting the individual profiles, which consisted of standardized T-scores on the Pros, Cons, and Confidence scales, allowed for a direct examination of the shape of the individual patterns within each stage and into what clusters these individuals were classified by cluster analysis. This graphical approach provides a detailed look at the accuracy with which the cluster analysis grouped similar individuals based on the three input variables.

Precontemplation. Figure 14 indicates that the majority of those in Precontemplation were clustered into the Disengaged and Early groups (32\% and $46 \%$ respectively). The individual profiles in these two clusters looked very similar. There 
were no precontemplators in the Maintainer cluster creating the only one of the 30 cells that was empty.

Contemplation. Figure 15 displays the profiles within each of the six clusters groups for individuals in the Contemplation stage. Fifty-eight percent of the Contemplators were in the Disengaged cluster. The remaining clusters each contained three or fewer contemplators. Because the Contemplation stage represented only about $8 \%$ of the total sample, it is not clear if other clusters would also represent this stage in a larger sample.

Preparation. The two clusters most associated with the Preparation stage were the Early (22\%) and Disengaged (39\%) clusters (see Figure 16). The Preparation stage is considered an unstable transition stage where individuals may be ready to move to Action or to regress back to an earlier stage. Finding more than $10 \%$ of those in Preparation in the At Risk, Early Action, and Habituated clusters is evidence for the instablity of the Preparation stage.

Action. The Action stage represented about $8 \%$ of the total sample and of all the stages, had the weakest association with the clusters. The majority of those in the Action stage were equally represented by the At Risk, Early Action, and Maintainer clusters (see Figure 17). Like the Preparation stage, the Action stage is also unstable in terms the high probability of stage movement and this is reflected by the three cluster patterns that primarily represent the stage.

Maintenance. The two clusters that were the most related to the Maintenance stage were the Maintainer and Habituated clusters, accounting for $52 \%$ of this stage (see 
Figure 18). Far fewer than expected Maintenance stage individuals were clustered into the Early and Disengaged clusters as indicated by standardized residuals of -3.1 and -2.6 respectively (see Table 18 ).

Plotting individual profiles is not commonly part of the cluster analysis procedure. Usually, only mean profile patterns of the clusters are examined. Plotting individual profiles by cluster stratified by stage of change, allowed for an examination of exactly "who" ended up in what cluster. The plots allowed for a visual inspection of the similarity of the grouped profiles, as well as where discrepancies existed. The greatest conformity of individual profiles seemed to be for the ' $\mathrm{V}$ ' shaped patterns (i.e. Early and Maintainers), while the Early Action and At Risk clusters seem to be the most heterogeneous groups. Visually comparing the densities of different plots indicates how closely a particular cluster was associated with a stage of change. For example, in Figure 18 , because there were a large number of individuals in the Maintenance stage, nearly all of the plots contained greater than ten individual profiles. However, the Maintainer and Habituated clusters clearly had the densest concentration of individual profiles, indicating that these clusters had the strongest association with the Maintenance stage. Although this procedure may not be practical when the sample size is exceedingly large, plotting individual profiles is an effective graphical approach to examining the heterogeneity of the $M$ clusters. 
Table 18

Two-Way Table for Exercise Clusters and Stage of Change for Exercise

\begin{tabular}{|c|c|c|c|c|c|c|}
\hline \multirow[b]{2}{*}{$\begin{array}{l}\text { Exercise } \\
\text { Clusters }\end{array}$} & \multicolumn{5}{|c|}{$\begin{array}{c}\text { Stage of Change: Exercise } \\
\text { (Frequency and Standarized Residual) }\end{array}$} & \multirow[b]{2}{*}{$\begin{array}{l}\text { Row } \\
\text { Total }\end{array}$} \\
\hline & PC & C & PR & A & $\mathrm{M}$ & \\
\hline \multirow[t]{2}{*}{ DI } & 20 & 15 & 29 & 4 & 32 & 100 \\
\hline & $(2.0)$ & $(2.7)$ & $(1.6)$ & $(-1.3)$ & $(-2.6)$ & \\
\hline \multirow[t]{2}{*}{ ER } & 14 & 3 & 16 & 3 & 7 & 43 \\
\hline & (3.6) & $(-.1)$ & $(2.2)$ & $(-.1)$ & $(-3.1)$ & \\
\hline \multirow[t]{2}{*}{$\mathrm{AR}$} & 3 & 2 & 8 & 6 & 20 & 39 \\
\hline & $(-.9)$ & $(-.6)$ & $(-.2)$ & $(1.8)$ & $(.1)$ & \\
\hline \multirow[t]{2}{*}{ EA } & 3 & 1 & 9 & 6 & 25 & 44 \\
\hline & $(-1.1)$ & $(-1.3)$ & $(-.2)$ & $(1.5)$ & (.6) & \\
\hline \multirow[t]{2}{*}{ MA } & 0 & 3 & 2 & 6 & 49 & 60 \\
\hline & $(-2.8)$ & $(-.7)$ & $(-3.0)$ & $(.7)$ & $(3.4)$ & \\
\hline \multirow[t]{2}{*}{$\mathrm{HA}$} & 4 & 2 & 10 & 1 & 39 & 56 \\
\hline & $(-1.2)$ & $(-1.1)$ & $(-.6)$ & $(-1.6)$ & $(2.0)$ & \\
\hline $\begin{array}{l}\text { Column } \\
\text { Total }\end{array}$ & 44 & 26 & 74 & 26 & 172 & 342 \\
\hline
\end{tabular}

Note. $\mathrm{PC}=$ Precontempation, $\mathrm{C}=$ Contemplation, $\mathrm{PR}=$ Preparation, $\mathrm{A}=$ Action, $\mathrm{M}=$ Maintenance. $\mathrm{DI}=$ Disengaged, $\mathrm{ER}=$ Early, $\mathrm{AR}=$ At Risk, $\mathrm{EA}=$ Early Action, $\mathrm{MA}=$ Maintainer, $\mathrm{HA}=$ Habituated. Chi-Square test of association $\left(\chi_{(20)}^{2}=98.8, p<.0001\right)$. 
Table 19

Relationship Between Clusters and Stages of Change

\begin{tabular}{|c|c|c|c|c|c|c|}
\hline & \multicolumn{5}{|c|}{ Stage of Change (Count and Percentage of Stage) } & \multirow[b]{2}{*}{ Cluster \% } \\
\hline Cluster & $\begin{array}{c}\mathrm{PC} \\
\mathbf{n}=\mathbf{4 4}\end{array}$ & $\begin{array}{c}C \\
n=26\end{array}$ & $\begin{array}{c}\text { PR } \\
n=74\end{array}$ & $\begin{array}{c}A \\
n=26\end{array}$ & $\begin{array}{c}M \\
n=172\end{array}$ & \\
\hline$+\infty$ & $\begin{array}{c}20 \\
46 \%\end{array}$ & $\begin{array}{c}15 \\
58 \%\end{array}$ & $\begin{array}{c}29 \\
39 \%\end{array}$ & & & $64 \%$ \\
\hline$\varepsilon=$ & $\begin{array}{c}14 \\
32 \%\end{array}$ & & $\begin{array}{c}16 \\
22 \%\end{array}$ & & & $70 \%$ \\
\hline Alik & & & & $\begin{array}{c}6 \\
23 \%\end{array}$ & & $15 \%$ \\
\hline - & & & & $\begin{array}{c}6 \\
23 \%\end{array}$ & & $14 \%$ \\
\hline & & & & $\begin{array}{c}6 \\
23 \%\end{array}$ & $\begin{array}{c}49 \\
29 \%\end{array}$ & $92 \%$ \\
\hline & & & & & $\begin{array}{c}39 \\
23 \%\end{array}$ & $70 \%$ \\
\hline $\begin{array}{l}\% \text { stage } \\
\% \text { sample }\end{array}$ & $78 \%$ & $58 \%$ & $61 \%$ & $69 \%$ & $52 \%$ & Total sample \\
\hline \% sample & $10 \%$ & $4.4 \%$ & $13 \%$ & $5.3 \%$ & $26 \%$ & $58.7 \%$ \\
\hline
\end{tabular}

Note. Cell percentages equal percentage of stage. 

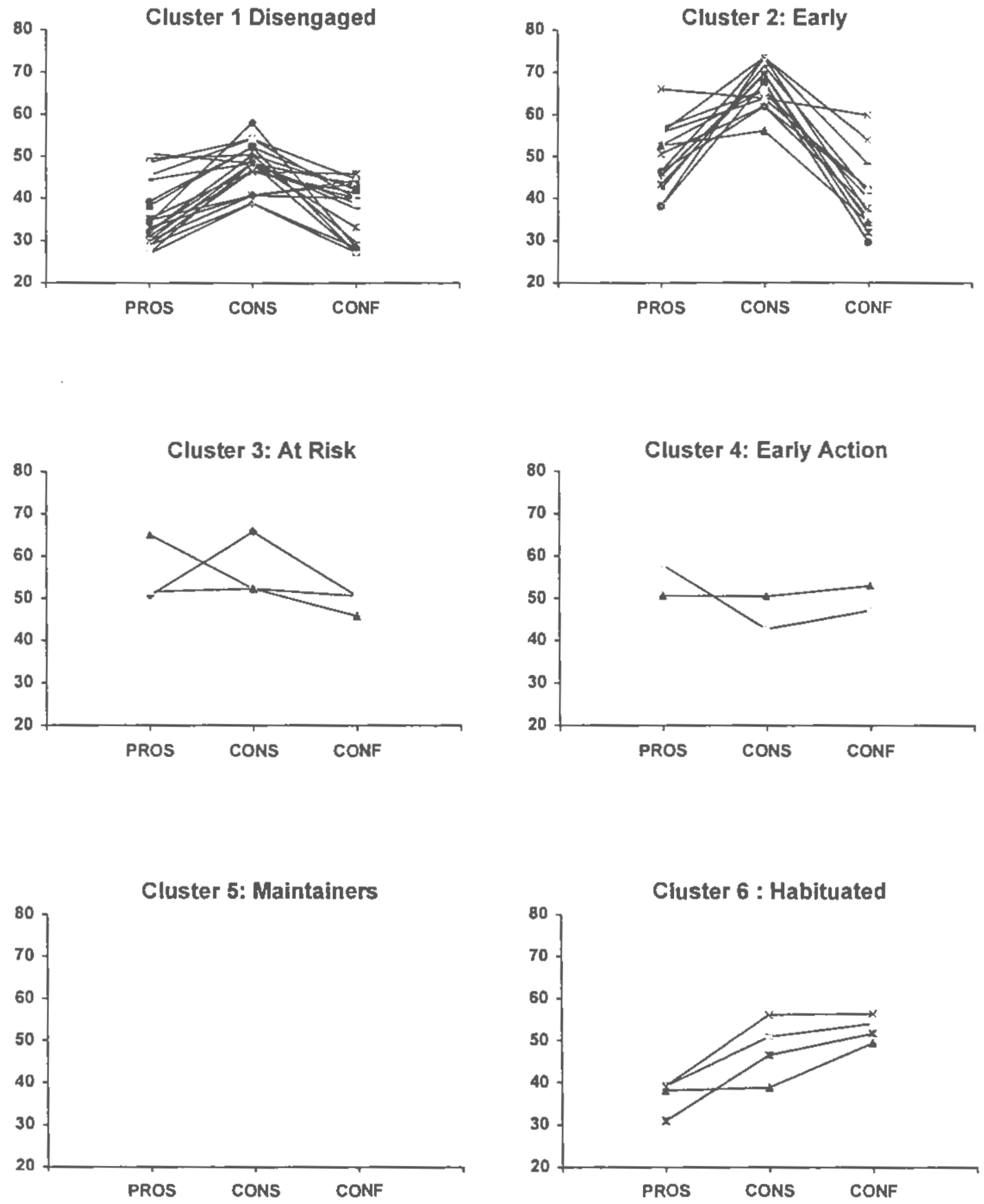

Figure 14. Individual profiles by cluster for the Precontemplation stage. 

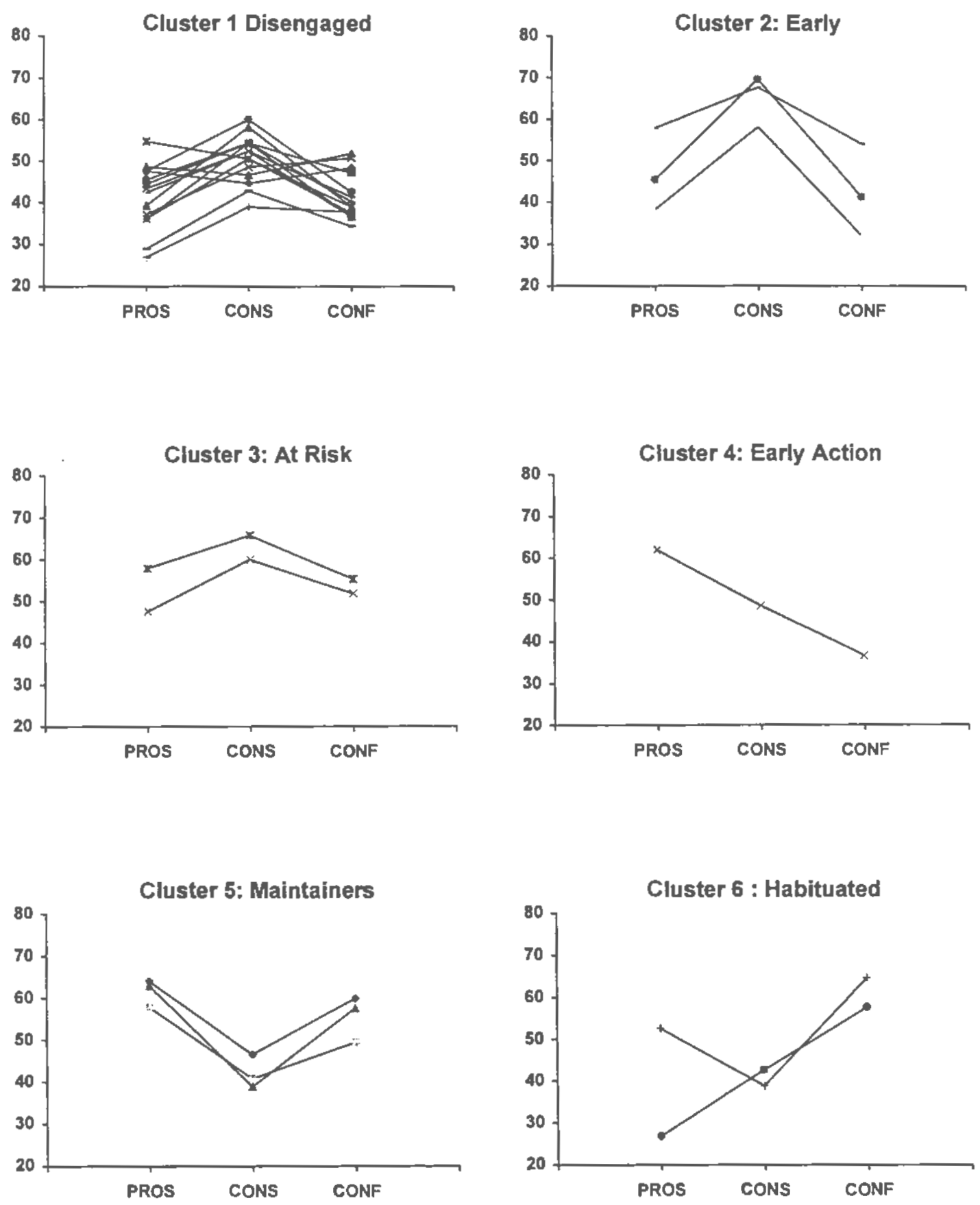

Figure 15. Individual profiles by cluster for the Contemplation stage. 

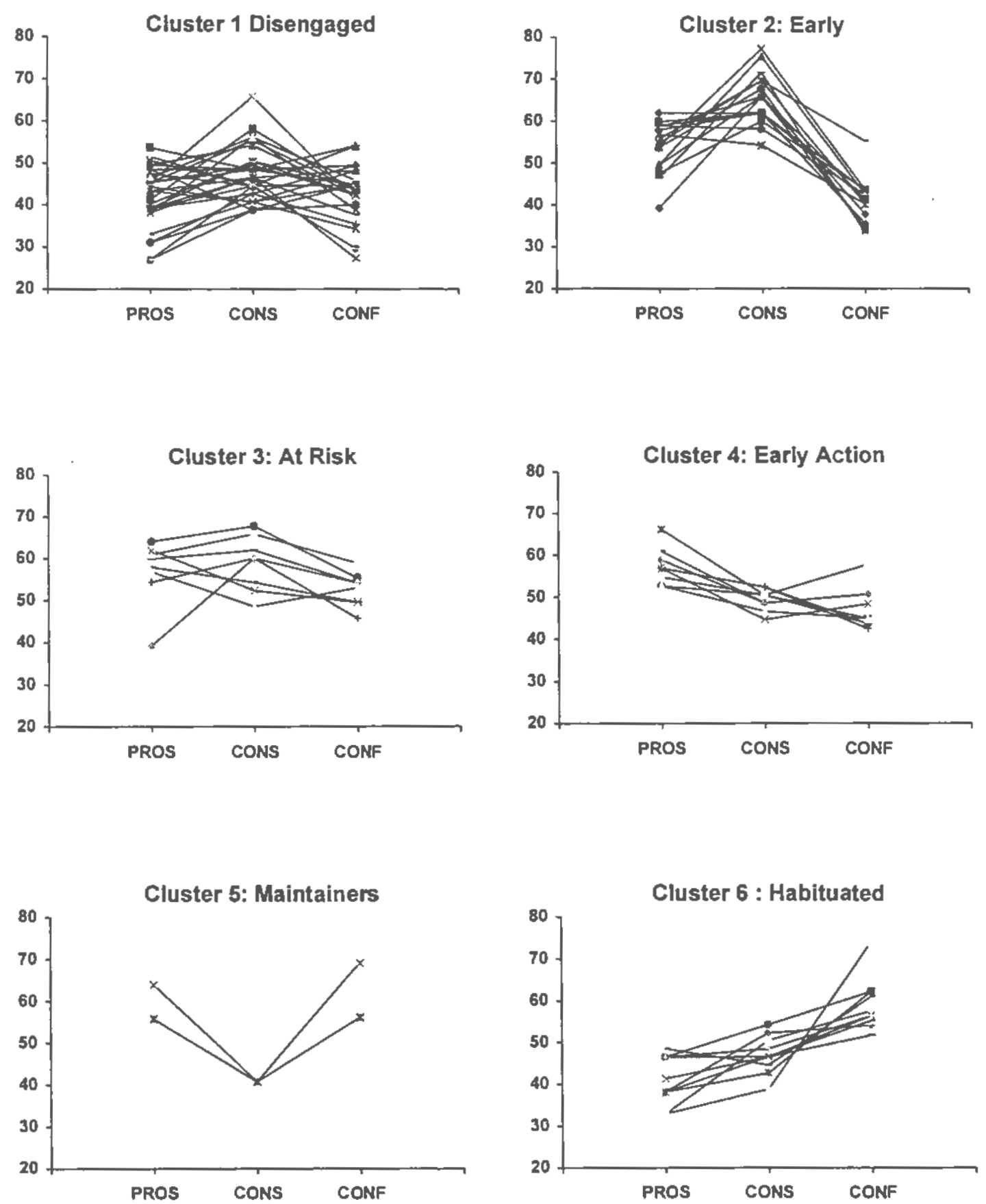

Figure 16. Individual profiles by cluster for the Preparation stage. 

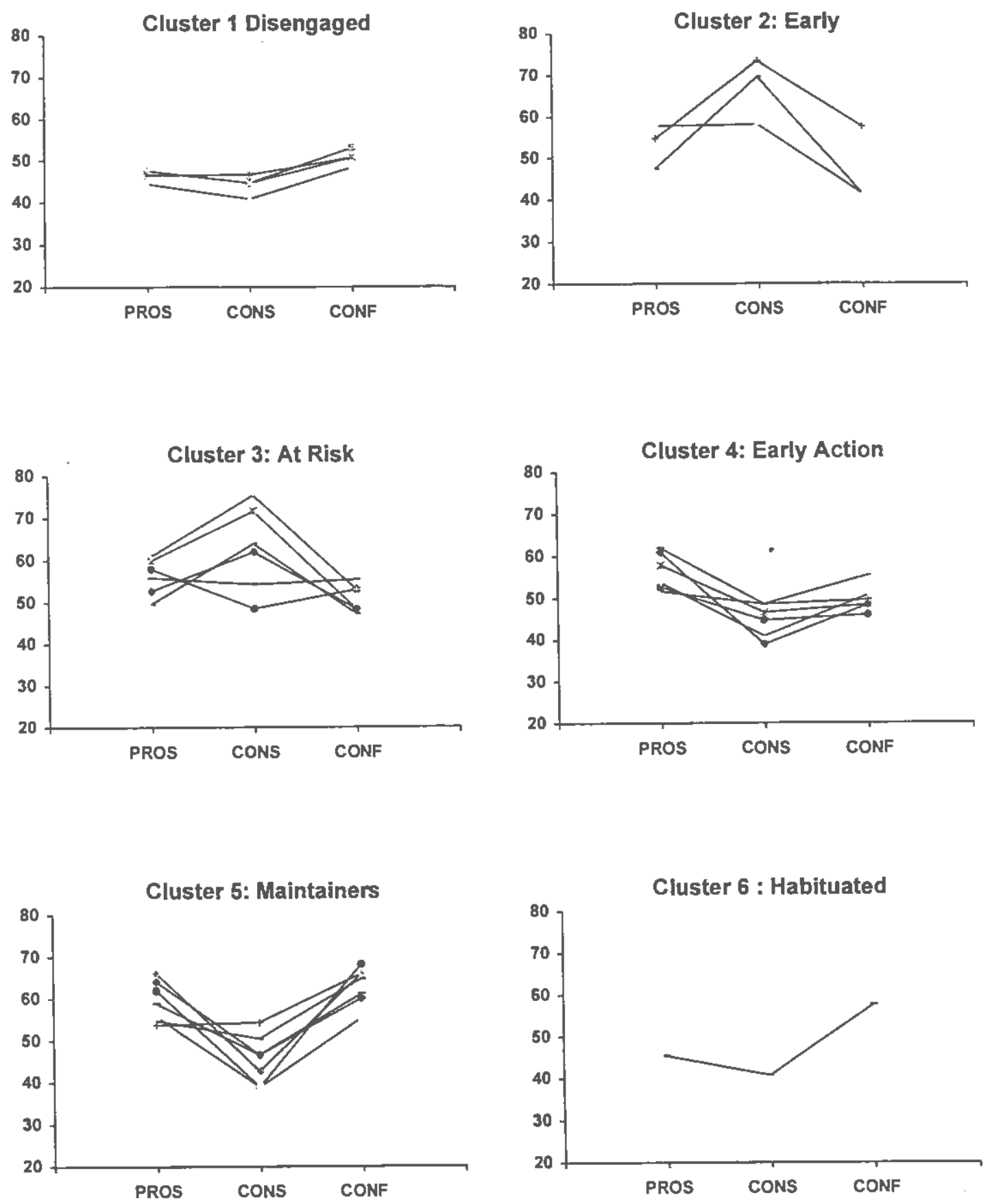

Figure 17. Individual profiles by cluster for the Action stage. 

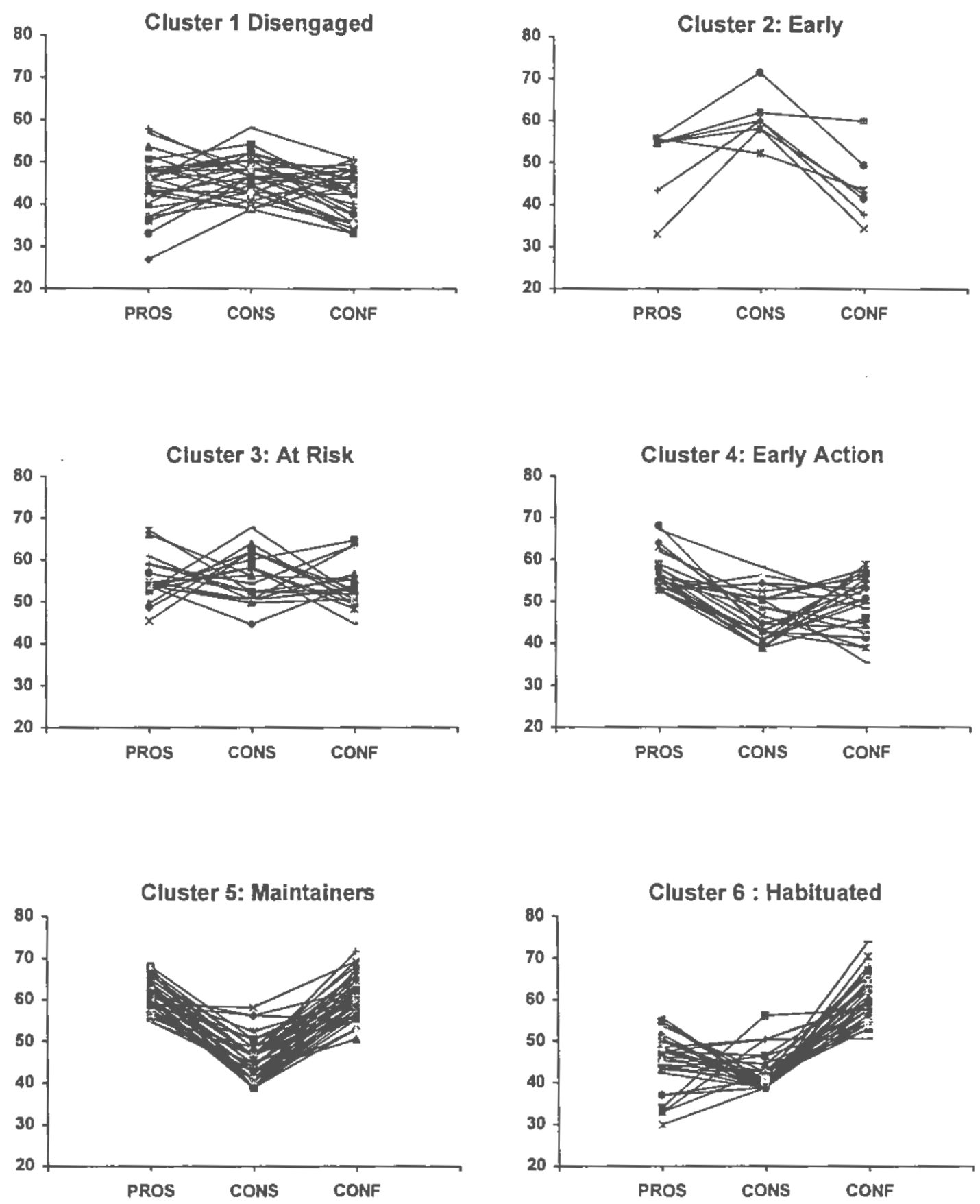

Figure 18. Individual profiles by cluster for the Maintenance stage. 


\section{External Validation: Stage of Change for Other Risk Factors}

In addition to exercise, the sample was assessed for their stage of readiness on three health risk behaviors: diet, sun protection, and cigarette smoking. These three categorical variables were used as independent variables in two-way contingency tables tested with Chi-square tests of independence. It was reasoned that individuals in more advanced clusters (e.g. Maintainers) would be more likely to be further along in the stages of change for other health risk behaviors. Such a relationship would indicate that the exercise clusters are related to other health risk behaviors.

Diet (fruit and vegetable consumption) and sun protection were assessed with single staging questions with five response categories corresponding to the five stages of change. To have adequate cell frequencies for the Chi-square analyses stage categories were collapsed from five to three by combining Action and Maintenance into a single category, and Contemplation and Preparation into a single category. The two 3 (stage groups) by 6 (clusters) chi-square tests indicated a statistically significant association between the exercise clusters and Diet stage of change $\left(\chi_{(10)}^{2}=28.90, p<.01\right)$ but not for Sun protection $\left(\chi_{(10)}^{2}=14.81\right.$ n.s.). The relationship between Diet staging and exercise clusters was in a positive direction with more Diet Action and Maintenance stage individuals in the Early Action and Maintainer clusters than expected and less Precontemplators in these same clusters than expected (see Table 20). The Habituated cluster had slightly more individuals in Precontemplation for Diet than expected. This finding indicated that although individuals in the Habituated cluster were exercising regularly, they seem to have less concern about eating a healthy diet. No clear pattern 
emerged between Stage of Change for Sun Protection and the exercise ciuster (see Table 21).

To assess the relationship between cigarette smoking and exercise clusters, the smoking stages of change were reduced to a point prevalence smoking status variable with two levels, current smoker (PC, C, PR) and non smoker (A, M, and never smoked). The collapsing of smoking stages was necessary to have adequate cell sizes for the analysis. The Chi-square test did not reach statistical significance $\left(\chi_{(5)}^{2}=10.55, \mathrm{p}=.06\right)$ but the trend was for less smokers than expected in the more active clusters (At Risk, Early Action, Maintainers, and Habituated) and more smokers than expected in the Disengaged and Early clusters (see Table 22). The opposite was true for nonsmokers, with more nonsmokers than expected in the active clusters and less than expected in the less active clusters. 
Table 20

Two-Way Table for Exercise Clusters and Stage of Change for Diet

Exercise Cluster Typology

(Frequency and Standarized Residual)

Stage of

DI ER AR EA MA

HA Row

Change:

Diet

\begin{tabular}{lccccccc}
\hline PC & 34 & 12 & 17 & 7 & 12 & 23 & 115 \\
& $(.6)$ & $(-.3)$ & $(1.4)$ & $(-1.8)$ & $(-1.5)$ & $(1.4)$ & \\
C \& PR & 40 & 22 & 12 & 16 & 20 & 11 & 121 \\
& $(.8)$ & $(1.7)$ & $(-.5)$ & $(.1)$ & $(-.3)$ & $(-2.0)$ & \\
A \& M & 25 & 9 & 10 & 21 & 28 & 22 & 115 \\
& $(-1.5)$ & $(-1.4)$ & $(-.9)$ & $(1.6)$ & $(1.7)$ & $(.7)$ & \\
Column & 99 & 43 & -39 & 44 & 60 & 56 & 341 \\
Total & & & & & & &
\end{tabular}

Note. $\mathrm{PC}=$ Precontempation, $\mathrm{C}=$ Contemplation, $\mathrm{PR}=$ Preparation, $\mathrm{A}=$ Action, $\mathrm{M}=$ Maintenance. $\mathrm{DI}=$ Disengaged, $\mathrm{ER}=$ Early, $\mathrm{AR}=\mathrm{At}$ Risk, $\mathrm{EA}=$ Early Action, $\mathrm{MA}=$ Maintainer, HA = Habituated. Chi-Square test of association $\left(\chi_{(10)}^{2}=28.9, \mathrm{p}<.01\right)$. 
Table 21

Two-Way Table for Exercise Clusters and Stage of Change for Sun Protection

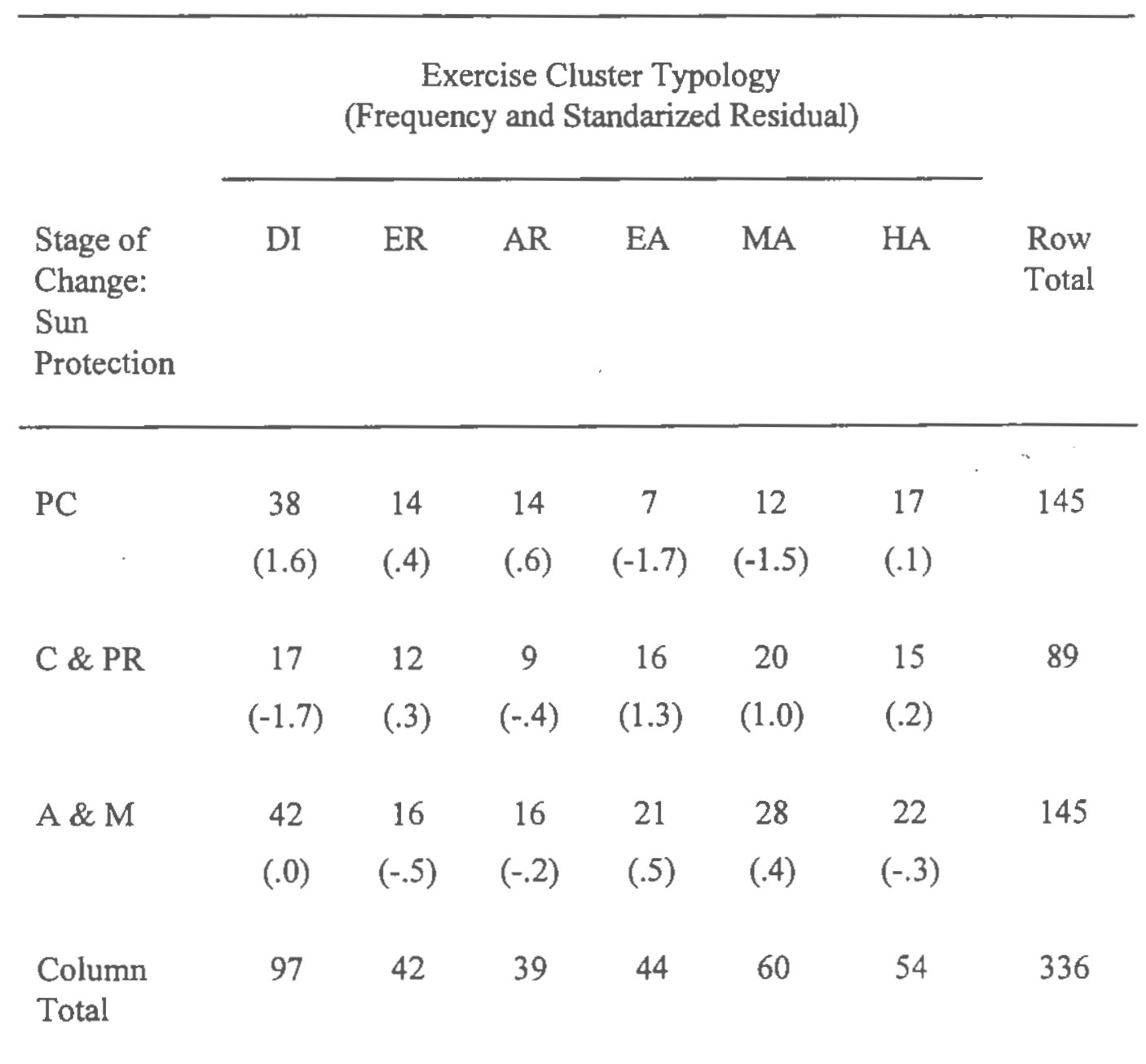

Note. $\mathrm{PC}=$ Precontempation, $\mathrm{C}=$ Contemplation, $\mathrm{PR}=$ Preparation, $\mathrm{A}=$ Action, $\mathrm{M}=$ Maintenance. $\mathrm{DI}=$ Disengaged, $\mathrm{ER}=$ Early, $\mathrm{AR}=$ At Risk, $\mathrm{EA}=$ Early Action, $\mathrm{MA}=$ Maintainer, $\mathrm{HA}=$ Habituated. Chi-Square test of association $\left(\chi^{2}{ }_{(10)}=14.81\right.$, n.s. $)$. 
Table 22

Two-Way Table for Exercise Clusters and Stage of Change for Smoking

Exercise Cluster Typology

(Frequency and Standarized Residual)

Stage of

DI ER AR EA MA

MA HA

Row

Change:

Smoking

PC, C, \& PR

25

135

5

8

8

64

(1.7) $\quad(-.9)$

$(-1.1)$

$(-1.0)$

$(-.7)$

A \& $M$

75
$(-.7)$

30

34

39

52

47

277

$(-.7)$

(.4)

(.5)

(.5)

(.3)

Column Tota

100

43

39

44

60

55

Note. $\mathrm{PC}=$ Precontempation, $\mathrm{C}=$ Contemplation, $\mathrm{PR}=$ Preparation, $\mathrm{A}=$ Action, $\mathrm{M}=$

Maintenance. $\mathrm{DI}=$ = Disengaged, $\mathrm{ER}=$ Early, $\mathrm{AR}=$ At Risk, $\mathrm{EA}=$ Early Action, $\mathrm{MA}=$

Maintainer, $\mathrm{HA}=$ Habituated. Chi-square test of association $\left(\chi_{(5)}^{2}=10.55, \mathrm{p}<.10\right)$. 


\section{External Validation: Demographics}

Relationships between the exercise cluster typology and several demographic variables were tested. These variables included: gender, education level, age, body mass index (BMI), and perceived health. Based on the exercise determinants literature, these variables have been associated with physical activity behavior (Dishman, 1990; King et al., 1992).

Gender was tested with a chi-square test of independence on the two-way contingency table of gender by exercise clusters. A statistically significant relationship was found for gender $\left(\chi_{(5)}^{2}=11.90, p<.05\right)$. More males and less females were in the Habituated cluster than expected, while more females and less males were found in the Early cluster than expected (see Table 23). This finding supports other studies reporting that males tend to be more regularly active than females.

The five categories of Perceived Health were reduced to three levels by combining the responses 'very good' and 'good' into a single category, and the responses 'fair' and 'poor' into one category. The third category was the response 'excellent'. Statistically significant relationships were found for the association between perceived health and the exercise clusters $\left(\chi_{(10)}^{2}=24.89, \mathrm{p}<.01\right)$ (see Table 24). People who rated their health as excellent were more likely than expected to be in the Maintainer cluster and less likely to be in the Disengaged and Early clusters. Those who rated their health as fair to poor were more likely than expected to be in the Early cluster and less likely to be in the Habituated cluster. These patterns of effects were evidence that general perceived health was related to the exercise clusters in the expected direction. 
Education level, age, and BMI were continuous dependent variables and tested in separate ANOVAs with exercise cluster membership as the independent variable. No statistically significant differences were found for the three dependent variables across exercise clusters (see Table 25). There was a trend for BMI, with lower mean BMI associated with more active clusters. Although this effect was not statistically significant the effect size was .029 . 
Table 23

Two-Way Table for Exercise Clusters and Gender

Exercise Cluster Typology

(Frequency and Standarized Residual)

Gender

DI ER AR EA MA

HA Row

Total

Female

$\begin{array}{lllll}55 & 33 & 22 & 28 & 40\end{array}$

26

204

$(-.6)$

(1.3)

$(-.3)$

(.2)

(.9)

$(-1.3)$

Male

42

10

16

16

17

29

130

(.7) $\quad(-1.6)$

(.3)

$(-.3)$

$(-1.1)$

(1.6)

Column Total

97

$43 \quad 38$

$44 \quad 57$

55

334

Note. $\mathrm{DI}=$ Disengaged, $\mathrm{ER}=$ Early, $\mathrm{AR}=$ At Risk, $\mathrm{EA}=$ Early Action, $\mathrm{MA}=$

Maintainer, $\mathrm{HA}=$ Habituated. Chi-square test of association $\left(\chi^{2}(5)=11.90, \mathrm{p}<.05\right)$. 
Table 24

Two-Way Table for Exercise Clusters and Perceived Health

Exercise Cluster Typology

(Frequency and Standarized Residual)

Perceived

Health

DI ER AR EA MA

HA Row

Total

$\begin{array}{lccccccc}\text { Excellent } & 15 & 5 & 6 & 13 & 19 & 16 & 74 \\ & (-1.4) & (-1.4) & (-.8) & (1.1) & (1.7) & (1.1) & \\ \begin{array}{lccccc}\text { Very Good - } \\ \text { Good }\end{array} & 75 & 31 & 28 & 30 & 39 & 40 & 243 \\ & (.5) & (.1) & (.1) & (-.2) & (-.6) & (0) & \\ \text { Fair - Poor } & 10 & 7 & 5 & 1 & 2 & 0 & 25 \\ & (1.0) & (2.2) & (1.3) & (-1.2) & (-1.1) & (-2.0) & \end{array}$

$\begin{array}{llllllll}\text { Column Total } & 100 & 43 & 39 & 44 & 60 & 56 & 342\end{array}$

Note. $\overline{\mathrm{DI}}=$ Disengaged, $\mathrm{ER}=$ Early, $\mathrm{AR}=$ At Risk, $\mathrm{EA}=$ Early Action, $\mathrm{MA}=$

Maintainer, HA $=$ Habituated. Chi-square test of association $\left(\chi^{2}{ }_{(10)}=24.98, \mathrm{p}<.01\right)$. 
Table 25

Descriptive Statistics and ANOVA Results for Demographic Variables by Exercise

Clusters

\begin{tabular}{|c|c|c|c|c|c|c|c|c|}
\hline \multirow[b]{2}{*}{ Variable } & \multicolumn{6}{|c|}{ Exercise Cluster Typology } & \multirow{2}{*}{$\begin{array}{l}\text { ANOVA } \\
\text { F - Value }\end{array}$} & \multirow{2}{*}{$\begin{array}{l}\text { Eta- } \\
\text { squared }\end{array}$} \\
\hline & DI & ER & $A R$ & EA & MA & HA & & \\
\hline Education & $\begin{array}{r}14.7 \\
(3.2)\end{array}$ & $\begin{array}{l}13.7 \\
(3.6)\end{array}$ & $\begin{array}{l}14.2 \\
(2.3)\end{array}$ & $\begin{array}{l}14.5 \\
(3.8)\end{array}$ & $\begin{array}{c}14.7 \\
(3.2)\end{array}$ & $\begin{array}{r}14.8 \\
(3.5)\end{array}$ & .90 & .013 \\
\hline Age & $\begin{array}{c}43.6 \\
(14.9)\end{array}$ & $\begin{array}{c}44.6 \\
(16.2)\end{array}$ & $\begin{array}{c}41.2 \\
(12.8)\end{array}$ & $\begin{array}{c}42.2 \\
(17.0)\end{array}$ & $\begin{array}{c}44.4 \\
(16.1)\end{array}$ & $\begin{array}{c}39.7 \\
(11.5)\end{array}$ & .92 & .013 \\
\hline BMI & $\begin{array}{l}26.2 \\
(4.3)\end{array}$ & $\begin{array}{l}27.7 \\
(5.9)\end{array}$ & $\begin{array}{l}27.2 \\
(5.6)\end{array}$ & $\begin{array}{l}25.6 \\
(3.7)\end{array}$ & $\begin{array}{l}25.8 \\
(5.0)\end{array}$ & $\begin{array}{l}25.2 \\
(3.8)\end{array}$ & 1.95 & .029 \\
\hline
\end{tabular}

Note. $\mathrm{BMI}=$ Body mass index. $\mathrm{DI}=$ Disengaged, $\mathrm{ER}=$ Early, $\mathrm{AR}=$ At Risk, $\mathrm{EA}=$ Early Action, $\mathrm{MA}=$ Maintainer, $\mathrm{HA}=$ Habituated. Degrees of freedom for all ANOVAs was $(5,335)$. 


\section{Discussion}

The discussion section is composed of five parts. The first three sections provide a summary of the results, address limitations of the study, and present future directions.. The fourth section titled, Implications for Interventions, links the results of the present study to a conceptualization of the TTM that is useful way to think about intervention development. Conclusions are drawn in the final section regarding testing the TTM and recommendations for using cluster analysis.

\section{Summary of Results}

An empirical typology of exercisers was created from a representative sample of adults. A series of internal and external validating analyses were conducted to determine the stability and distinctiveness of the typology's cluster groups. Six cluster groups were found through cluster analysis based on three key variables from the Transtheoretical Model: the Pros and Cons of exercise, and Exercise Confidence. As a result, the clusters represented groups which differed in respect to confidence in their ability to exercise regularly and on the importance of the positive and negative aspects of exercise in their decision to exercise regularly. The internal validation analyses revealed that four of the six clusters consistently replicated while two clusters replicated inconsistently. In the external validation analyses it was hypothesized that these groups would be related to self reported exercise behavior, use of the 10 processes of change, stage of change, as well as certain demographic and health related variables. External validation of the clusters in relation to stage of change revealed that each cluster represented individuals primarily 
found in one or two adjacent stages. A major conclusion from this series of studies was that the six clusters represent a limited number of stage subtypes similar to subtypes found for cigarette smoking behavior when empirical typologies have been created within the stages of change (Velicer et al., 1995; Norman et al., 1997). However, it is not clear if the six clusters found in the present study represent an exhaustive set of exercise subtypes.

This section summarizes the internal and external validation analyses. To facilitate this, the six clusters were grouped into three sets of two clusters. Each cluster seemed to have a nearest neighbor in terms of similarity of certain characteristics. These cluster pairs are the Disengaged and Early; the At Risk and Early Action; and the Maintainers and Habituated. Table 26 provides a brief summary of the results which are discussed below.

Disengaged and Early. The first two clusters, the Disengaged and Early, were exercising below the criteria for regular exercise. These cluster profiles represented individuals who were not confident about their ability to exercise regularly and reported that the Pros of exercise were not important to them, compared to other cluster groups. What distinguished these two groups was the Cons of exercise. The Early group reported high Cons while the Disengaged group reported low Cons. The difference in the Cons between the two groups may reflect the Disengaged group's lack of awareness and/or experience with the negative aspects of regular exercise. The Early cluster group may have attempted to exercise regularly in the recent past but found that the Cons of exercising were too difficult a barrier to overcome. The Disengaged cluster's flat profile 
pattern with low elevation has been found previously in empirical typologies of smokers (Velicer et al. 1995; Norman et al., 1997). However, this profile shape has a different interpretation in exercise because confidence is low, but in smoking it is temptation to smoke that is low. However, the implications for disengaged groups may be similar in terms of reaching these individuals. They may be difficult to motivate and are not likely to sign up for, or participate in change programs that rely on reactive recruitment.

The Disengaged cluster was mostly comprised of individuals from the three early stages of change. The cluster represents an important early stage subtype not hypothesized within the TTM. The Early cluster pattern profile was predicted to be found as representing primarily Precontemplation stage individuals. However, this cluster also represented people in the Preparation stage. Both the Disengaged and Early clusters demonstrated high cluster stability and replicability in the random subsample clustering and the cross-validation analyses, indicating that these were two distinct patterns that would be expected to be found in future studies.

Process of change use between these two groups did differ somewhat on the Experiential processes but did not differ on the Behavioral processes. The only statistically significant process difference between the two clusters was on Self Reevaluation. However, the average process use for the Early cluster was almost a half standard deviation higher than the Disengaged cluster on the four remaining experiential processes. This finding supports the relationship posited by the TTM that experiential processes differentiate early stage individuals and behavioral process use does not (Prochaska et al., 1988; Prochaska \& Marcus, 1995). Interestingly, although not 
statistically significant, the Disengaged cluster was engaging in more exercise activity than the Early cluster. This may indicate that individuals in the Disengaged cluster exercised sporadically but have not given much thought or commitment to making regular exercise a part of their lives.

At Risk and Early Action. The At Risk and Early Action profiles represented individuals primarily in the Action stage of change. These were the two clusters that were unique to the original individual cluster analysis subsamples. Aside from differences in profile shape and scatter, there was little internal validation evidence and no external validation evidence to indicate that these two clusters were distinct. The random subsample replication test demonstrated that these were the two cluster patterns that were least likely to be replicated from the data. However, the assignment discrepancies found in the double cross-validation analysis did indicate that individuals in the At Risk cluster were likely to be assigned to the Early cluster, while individuals in the Early Action cluster was likely to be assigned to the Maintainer cluster. Exercise activity level and process of change use were equivalent for the two clusters in the external validation tests.

The flat profile with average elevation of the At Risk group was hypothesized initially to represent earlier stage individuals, particularly contemplators. This hypothesis was based on empirical typologies of smokers. Findings from the present study indicated that the Cons remain higher than the Pros into the Action stage. The cross-over effect of the Pros and Cons is delayed until the Action stage. This may be an important distinction between an acquisition behavior change such as exercise and a cessation behavior change 
such as smoking.

Although almost no supportive evidence was found to warrant keeping the At Risk and Early Action clusters separate, they were important in the overall analysis because they represented an interesting comparison with the other clusters across the different validating analyses. Initially, these clusters appeared distinct based on differences in profile characteristics. Thus, they passed the first level of the analysis. Subsequent validating analyses indicated no discernible distinction between the two cluster groups. Combining these two groups would result in a cluster with a flat profile with low scatter and elevation somewhat above average. Although no validating evidence was found in the present study to indicate that these were two distinct clusters, they should not be dismissed until future studies can test for their existence in other samples.

Maintainer and Habituated. Individuals in these two clusters reported being very confident in their ability to exercise regularly and did not rate the Cons of exercise as very important in their decision to exercise regularly. What differentiated these two clusters was the Pros of exercise. The Maintainer cluster reported high Pros while the Habituated cluster reported low Pros. These two clusters were most closely associated with the Maintenance stage of change, with $70 \%$ of the Habituated cluster in the Maintenance stage. Because these clusters were both associated with the later stages of change, the low Pros of the Habituated cluster was interpreted as indicating that individuals in this cluster engaged in little cognition regarding their decision to exercise, they just did it. Hence, they were labeled Habituated, because exercise was a well formed 
habit. The overall level of exercise did not differ between the two clusters on the composite Godin scale, but the Maintainer cluster reported more moderate level exercise (e.g. fast walking, easy bicycling) than the Habituated cluster. Process use for the Habituated cluster was significantly lower for all ten processes compared to the Maintainer group. The pattern of process use for the Habituated cluster resembled the first two clusters (Disengaged and Early) for the experiential processes, but behavioral process use more closely resembled the At Risk and Early Action clusters. This information, taken together, indicated that the Habituated cluster group was engaging in regular exercise but not reporting the kinds of cognitions and strategies hypothesized to be associated with successfully maintaining a program of regular exercise.

Because this was a cross-sectional study, there is only concurrent validity evidence to support the conjecture that the Habituated cluster represents the equivalent of the Termination stage in smoking (Prochaska, 1996). That is, it is believed that this is a group who have made regular exerciser a habit in their lives. This conjecture can only be tested in a naturalistic prospective study where it would be hypothesized that the Habituated group would be less likely to consist of individuals who relapse from their exercise habit than would a group of Maintainers or other regularly active group. On the other hand, the Habituated group may represent individuals who are part of a structured exercise environment such as a tennis league, an aerobics class, or a local running group. The structure allows these individuals to exercise regularly without having to rely on selfmotivation. If this is the case, then the Habituated cluster may be dependent on that social structure and not have important self-controls in place to maintain their level of 
exercise. Only further study of individuals who report being regularly active will provide information about these different groups' regular exercise behavior.

Below is a final summary of the results.

1) An empirical typology of exercisers consisting of six clusters was found in a random sample of surveyed adults.

2) The six clusters were interpretable within the Transtheoretical model as stage of change subtypes. The clusters related closely to the five stages of change.

3) Internal validation, using random subsample clustering and double cross-validation, indicated that four of the cluster groups were highly stable and replicable and two clusters replicated inconsistently.

4) External validation using self reported exercise behavior and physical activity indicated that clusters differed on strenuous and moderate levels of exercise (effect sizes of .09 and .07 respectively) but not on mild exercise or measures of physical activity. 5) External validation using the processes of change indicated large differences between clusters with effect sizes ranging from .13 to .32 .

6) External validation with risk factors of Diet, sun exposure, and cigarette smoking found only a relationship with Diet stage of change and the exercise clusters.

7) External validation using several demographic variables found cluster relationships between gender and perceived health but not relationships between clusters and education, age, and BMI. 


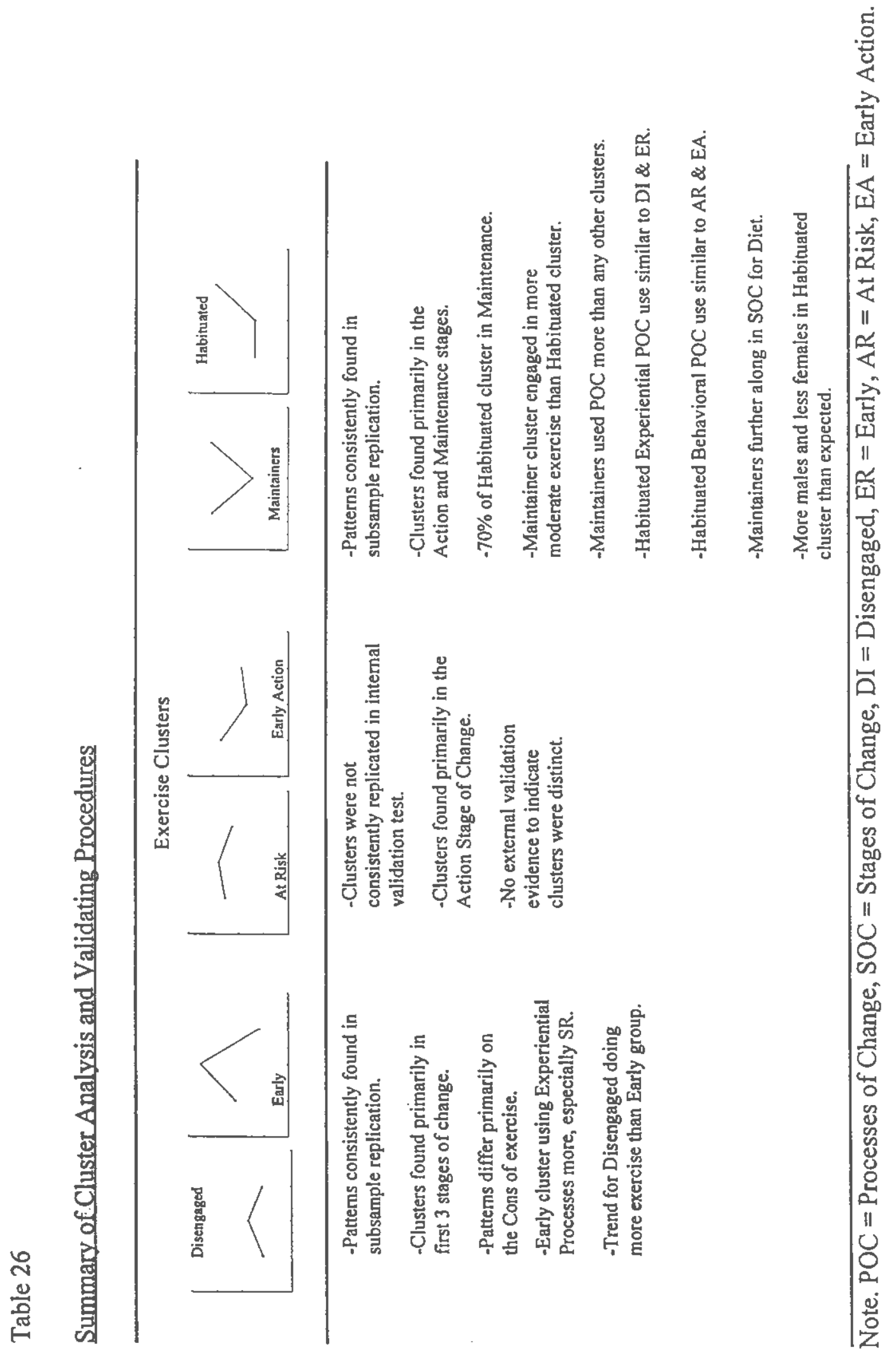




\section{Limitations of the Study}

Several important study limitations need to be addressed. These issues include the limits of a cross-sectional study, the limitations of cluster analysis, participant information that was not assessed, and the physical activity level of the overall sample.

First, this was a cross-sectional study where participants were anonymously contacted and no follow-up was planned or possible. Having a cross-sectional sample limited what can be learned about the construct relationships. While concurrent validity could be established for the cluster typology, only conjectures could be made from this data about exercise behavior change over time.

Second, similar to any statistical method, it is important to recognize the limitations of the cluster analysis procedure. No statistical procedure is self-interpreting and cluster analysis is no exception (Waller \& Meehl, 1998). Cluster analysis is a descriptive procedure that relies on the quality of the data rather than on theoretical probabilities to determine the meaningfulness of the results. Cluster analysis will always provide a solution, and therefore will always form groups within the data. By using a number of internal and external validating procedures, a convergence of evidence determines the verisimilitude of the cluster solution. This was achieved in the present study. Replicating the typology in other samples will further validate the exercise clusters.

Third, in retrospect, there were a few important questions that were not asked of the participants. These included gathering information about the kinds of exercises and physical activities in which the participants actually engaged. For comparative purposes, 
it would have been beneficial to have staged individuals for moderate intensity physical activity behavior.

A fourth concern of the study was that the sample, although randomly selected, was more physically active than is representative of the population. Fifty percent of the sample reported being in the Maintenance stage of change for regular exercise, which is higher than the $30 \%$ to $40 \%$ range that has been reported in other studies (Marcus \& Owen, 1992; Marcus, Selby et al., 1992; Reed et al., 1997), although Reed et al. (1997) did report that $52 \%$ of participants in a sample of convience $(n=306)$ were in the Maintenance stage for vigorous exercise. The higher proportion of active people in the present study may reflect a difference between worksite samples, in which many exercise studies are based, and a general population sample. There is also the possibility of a selection bias with more active individuals willing to participate in a survey on exercise behavior. However, the $68 \%$ response rate indicated that the sample was a reasonable random selection of the population. It should also be noted that from 1990 to 1994 the percentage of adults classified as sedentary in Rhode Island was lower than the United States median in each year (Hesser \& Kim, 1997). Recent unpublished data from the Behavioral Risk Factor Surveillance System for the state of Rhode Island indicated that $45 \%$ of Rhode Islanders engage in regular leisure-time physical activity. Based on the results of the study, there is no reason to believe that the participants inaccurately reported their levels of exercise and physical activity. A less physically active sample may have led to potentially more cluster groups representing subtypes in the earlier stages of change. 


\section{Future Directions}

The exercise clusters found in the present study provide the evidence of interpretable cluster groups based on key Transtheoretical model variables. Future studies are warranted that can test the replicability of the cluster groups both across and within stages of change for regular exercise. It will be important to assess the presence of these clusters in samples that vary in terms of overall level of exercise and specific subgroups such as youth, elderly, and ethnically diverse samples. Larger samples will be needed to perform cluster analysis within stages of change. Sampling stage groups consisting of approximately 100 individuals will allow for clustering and cluster validation tests within each of the stages of change. Prospective study designs are also needed to examine cluster groups over time. Cluster groupings formed at a baseline assessment and followed up over several time points could be used in longitudinal analyses such as dynamic typologies (Prochaska, et al., 1991; Norman, et al., 1997) or latent growth curve modeling (McArdle \& Epstein, 1987; Raykov, 1997) to examine change over time. Such analyses are crucial for determining if the exercise clusters can serve as targeting groups for clinic or population based interventions aimed to promote and maintain exercise behavior.

Another important direction to consider is the application of this series of analyses to other health behaviors where Transtheoretical model research has been conducted. As has been demonstrated in this study, cluster analysis can be a useful procedure for assessing a group of theoretically related constructs and serves both exploratory and 
confirmatory purposes. Variable patterns and relationships from the data, which may not have been hypothesized within the original theory, can potentially be discovered. Although, much of the initial empirical support for the TTM is based on smoking cessation research, the constructs that were developed from this research mapped well onto exercise behavior providing evidence that the TTM can be considered a general model of behavior change. However, finding Disengaged and Habituated clusters added new possibilities to better understanding the process of adopting and maintaining regular exercise. Complex behavior changes related to diet and sun protection may be better understood through creating theoretically based empirical typologies with cluster analysis. The results of the present study indicate that mapping the TTM to a new behavior does not allow for determining unique aspects of a particular kind of change which may be specific to a single behavior or general to a group of behaviors, such as acquisition behaviors like exercise.

\section{Implications for Interventions}

The Transtheoretical model (TTM) can be conceptualized as three tiers of constructs: 1) stages of change, 2) the dependent outcome variables (pros, cons, confidence, and behavior), and 3) the ten independent processes of change. Each of the three tiers of the model represents important components in the change process. The stages represent intention and readiness to change. The outcome variables at level 2 reflect cognitions and evaluations about the behavior. The processes at level 3 are the cognitive and behavioral strategies commonly used by individuals to facilitate and 
maintain change. This level configuration of the model creates a convenient way for conceptualizing how the theoretical constructs can be used to develop interventions based on the Transtheoretical Model. Exercise will be employed as an example.

In the present study, the focus was on the dependent outcome variables at the second level of the model. An effort was made to find groups of individuals based on the similarity of these measures. The clusters that were found can serve as subtypes to develop and test targeted interventions. Subtypes were predicted to be closely related to the stages of change. Evidence for this hypothesis was found in the present study. A number of predictable cluster profile patterns based on the pros, cons and confidence were found. These patterns were related to specific stages of change. Cluster groups were also found that were not predicted but were interpretable within the TTM. Evidence for the existence of exercise subtypes warrants conceptualizing how they can be used to develop effective interventions.

The rest of this section further describes the three levels of.the TTM and how interventions can be developed based on the variables at these levels. Particular attention is paid to the second level which was the focus of the present study.

At the first level is stage of change, five discrete categories representing individuals' readiness to adopt or maintain a behavior change. The stage categories are a temporal ordering reflecting intention to engage in a behavior and an indication of how long an individual has been engaging in the behavior. Other TTM variables have shown predictable relationships across stages indicating when these variables are relevant to the change process and when they are not. 
At the second level are constructs which represent the multivariate outcome space. Interventions developed at this level fall in-between the stage tailored and the completely individualized approaches. Currently there are no examples of interventions developed at this level but interesting possibilities exist. The three key constructs at Level 2 are labeled Positive Evaluation Strength, Negative Evaluation Strength and Habit Strength. These constructs are fully described by Velicer et al. (1996) in the Criterion Measurement Model (CMM). The CMM provides a multivariate continuous assessment of behavior change in place of traditional dichotomous outcome variables such as point prevalence.

The continuous outcome measures describe the movement of individuals through the change process from the Precontemplation stage to the Maintenance stage. For exercise, exemplary measures of Positive Evaluation Strength and Negative Evaluation Strength come from the Pros and Cons of change scales from the Exercise Decisional Balance Inventory and the Exercise Confidence Inventory. Measures of exercise behavior such as the Godin Leisure Time Exercise Scale, are also important outcome measures. The CMM combined with the stages of change provide a detailed assessment of where an individual is in the change process.

The ten processes of change at Level 3 are strategies commonly used by individuals to make life changes. The use of different processes varies across the stages of change and empirical research has determined when different processes should be used to facilitate change. Because different processes are important during different stages, individuals can be given suggestions and feedback on just the processes they need for where they are in the change process. 
For each of the three levels different kinds of interventions can be created that vary from a broader group focus to a narrower individualized focus. Figure 19 displays how the three levels of the model branch to create multiple types of interventions. This depiction is that of a hierarchical model where each subsequent level can include the previous levels.

Interventions can vary in terms of the amount of individualized feedback and normed matched information that is delivered. The length of the assessment to generate feedback increases as interventions are created at higher levels. At Level 1, interventions deliver individualized feedback about one's stage of change along with staged matched general educational information about the constructs at the subsequent levels of the model. At Level 2, feedback can be provided about one's stage and one's subtype along with normed matched information about the processes of change. At Level 3 interventions can be designed that provide individualized feedback on constructs at all three levels.

Figure 19 begins at the Group Level with a single intervention. Typically at the Group Level, an action-oriented treatment is provided to all participants regardless of their readiness to change. In these types of interventions the participants are expected to conform to the intervention rather than the intervention meeting the needs of the participants. The end result of the Group Level intervention is that it has little impact due to low participant retention and minimal long term progress (Prochaska, 1996).

At Level 1, a relatively small number of tailored interventions can be created. The tailored level moves away from the Group Level's single treatment approach. 
Velicer, Rossi, Ruggiero, and Prochaska (1994) describe a set of stage of change tailored manuals for smoking cessation. Tailored intervention systems have also been designed for making dietary changes and mammography screening (Skinner, Strecher, \& Hospers, 1994; Strecher et al., 1994).

At Level 2, a larger number of interventions can be developed that can be targeted to the stage subtypes. Adding subtype level information to stage of change multiplies the number of potential interventions by a factor of four or five. Once individuals' stage of change is identified, they could then be assigned to the subtype that is closest to their multivariate outcome profile.

The subtype level of targeted interventions is where the least emphasis has been placed in the area of TTM related intervention development and evaluation. Using cluster subtype groups combined with stage of change could be used to create a series of targeted interventions that focus on increasing individuals awareness about their attitudes and beliefs regarding exercise. An intervention message to early stage individuals who are in the Disengaged cluster might be to focus more on how regular exercise would benefit their lives. At the same time they need to know that this process will also likely increase their awareness of the barriers (Cons) to exercise. These individuals can then be reassured that they can get help to effectively minimize these barriers as they progress through the change process.

Creating targeted interventions using subtypes may be very useful from a cost effectiveness perspective. Less time could be taken in the assessment because the processes of change do not have to be measured. Targeted interventions could contain 
general feedback concerning strategies but interactive feedback based on the stage and subtype assessment. Shortened assessment and intervention delivery times may be of importance for multiple risk assessments. For example, several behaviors such as exercise, diet, and smoking may be the focus of a behavioral intervention for treatment of hypertension. Targeted interventions using exercise subtypes found in the present study offer a new direction for creating TTM based interventions.

At Level 3, the ten processes of change are added to create literally thousands of individualized interventions. Specific feedback about the different change strategies through individualized interventions can appropriately be handled through computerized expert system technology (Velicer, Prochaska et al., 1993). Based on structured assessments, the computer determines and delivers the appropriate message to the individual. Examples of computer-based interventions at this level have been developed for smoking cessation (Pallonen et al., 1998; Ruggiero, Redding, Rossi, \& Prochaska, 1997) and skin cancer prevention (Rossi, Weinstock, Redding, \& Cottrill, 1996).

Thus, three levels of the model can be used to develop either stage tailored, subtype targeted, or completely individualized interventions. The factors that determine the appropriate level to use for developing interventions include, but are not limited to: (1) the medium in which the intervention will be delivered, (2) the population that will be receiving the intervention, (3) the resources available to create and disseminate the intervention, and (4) the dosage of intervention believed to be necessary to be effective. Different combinations of interventions can also be given in a step-care design to maximize cost effectiveness. It may also be of interest to impact on a community through multiple channels employing combinations of intervention levels. For example, 
a population wide campaign to increase exercise behavior could use stage tailored mass media messages in conjunction with targeted subtype level pamphlets delivered through the mail. 


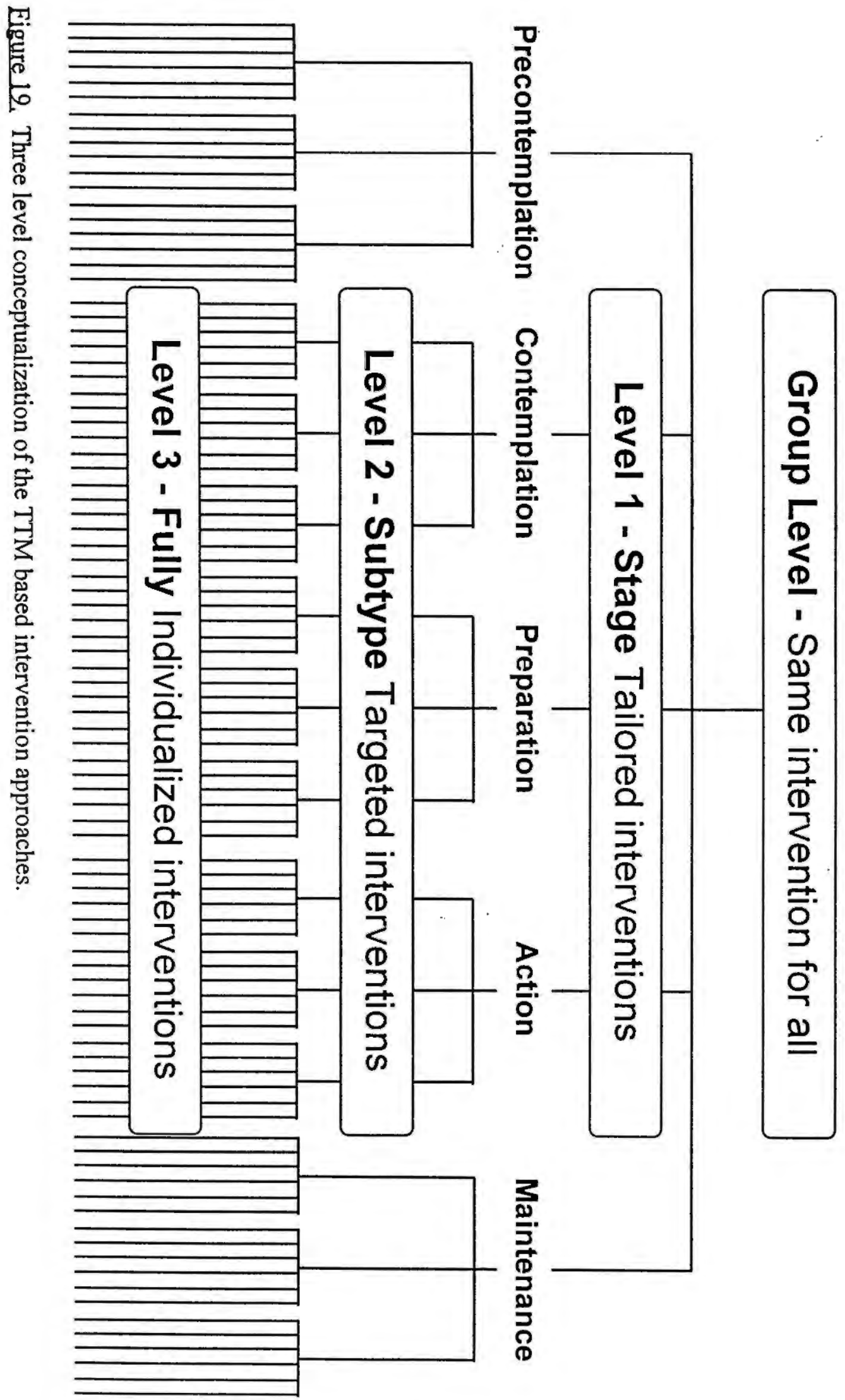




\section{Conclusions}

Testing the TTM. The present study began with the investigation of the problem of understanding how individuals change their behavior from not exercising to exercising regularly. The proposed solution is the theory. In this case it was the Transtheoretical model of behavior change. In this theory, it is hypothesized that individual change can be understood as movement through a series of stages and that certain attitudes, beliefs, and use of change strategies vary systematically across these stages. In the present study, a representative cross-section of individuals was examined using cluster analysis to determine what kinds of homogeneous groups existed. It was hypothesized that if a set of clusters could be found that could be interpreted within the theory, then the resulting empirical typology was supporting evidence for the theory. If no clusters, or a set of clusters that was not related to the theory was found, then this would be evidence against the theory's generalization to exercise behavior.

The empirical typology developed in this study was interpretable within the TTM. A central postulate of the TTM is that changing behavior involves progressing through a sequence of stages. The exercise typology, although it does not have a one to one correspondence between clusters and stages of change, does not refute the postulate that individuals change their exercise behavior through a series of stages. Rather, the clusters were interpreted as representing a limited number of stage subtypes within each of the stages of change but further studies are needed to support this conjecture. The available evidence can be interpreted as supporting the hypothesis that discrete groups can 
represent different types of individuals across the entire change process, and that behavior change can be modeled as more than simply a binary, 'all or nothing', process.

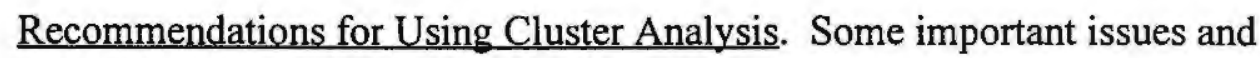
recommendations concerning the use of cluster analysis were addressed in this study that may be helpful to users of cluster analysis. First, beginning with a well formulated theory facilitated the cluster analysis procedure at many levels. Particularly, theory guided the variable selection, facilitated determining the number of clusters, and guided the external validation process. When used without theory, cluster analysis is purely an exploratory procedure, but when used with theory it can serve exploratory and confirmatory purposes.

Second, with an actual sample the true number of clusters can never really be known, compared to simulated data where the true cluster membership is known a priori. Using theory and previous research allowed for hypothesizing what some of the cluster profiles should look like, which provided guidance for finding a cluster solution. As a result, the analysis focused on finding reliable cluster profiles rather than simply determining the number of clusters. In the present study a limited range of plausible cluster solutions were explored instead of finding and interpreting a single solution.

Third, fast computers and easy to use graphics programs can aid in exploring and presenting the results of a cluster analysis. Graphical displays are recommended for all phases of the cluster analysis, from dendrogram interpretation, to comparing cluster profiles, to plotting external validating variables across cluster groups.

Finally, cluster analysis does not replace traditional statistical tests but should be used in conjunction with them. A convergence of evidence should be sought rather than 
relying on a single statistical test. Using the internal and external analyses allowed for developing a more complete understanding of the data and the variable relationships. 
Appendix 
Please:

- Use a * 2 dencil.

- Fill in each circle completely.

- Erase all chanoes completelv.

- Do not make any stray marks on this form.

\begin{tabular}{|c|c|}
\hline $\begin{array}{c}\text { (Interviewers: record bv observation) } \\
\text { Gender: }\end{array}$ \\
\hline $\begin{array}{c}\text { Female } \\
\text { O }\end{array}$ & Male \\
& $\bigcirc$ \\
\hline
\end{tabular}

\begin{tabular}{|c|c|c|c|c|c|c|c|}
\hline \multicolumn{8}{|c|}{ Subject in } \\
\hline 0 & 0 & 0 & 0 & 0 & 0 & 0 & 0 \\
\hline 1 & 0 & 1 & 0 & 1 & 0 & 1 & 0 \\
\hline 2 & 0 & 2 & 0 & 2 & 0 & 2 & 0 \\
\hline 3 & 0 & 3 & 0 & 3 & 0 & 3 & 0 \\
\hline 4 & 0 & 4 & 0 & 4 & 0 & 4 & 0 \\
\hline 5 & 0 & 5 & 0 & 5 & 0 & 5 & 0 \\
\hline 6 & 0 & 6 & 0 & 6 & 0 & 6 & 0 \\
\hline 7 & 0 & 7 & 0 & 7 & 0 & 7 & 0 \\
\hline 8 & 0 & 8 & 0 & 8 & 0 & 8 & 0 \\
\hline 9 & 0 & 9 & 0 & 9 & 0 & 9 & 0 \\
\hline
\end{tabular}

\section{GENERAL HEALTH QUESTIONS}

1. Would vou say your health in general is (read all choices):

\begin{tabular}{|ll|ll|ll|ll|ll}
\hline 0 & 1Excellent & $\mathrm{O}$ & 2. Very Good & $\mathrm{O}$ & 3. Good & $\mathrm{O}$ & 4. Fair & $\mathrm{O}$ & 5. Poor \\
\hline
\end{tabular}

\begin{tabular}{|l|ll|ll|}
\hline 2. Do you consider yourself overweight? & $\mathrm{O}$ & 1. No & $\mathrm{O}$ & 2. Yes \\
\hline
\end{tabular}

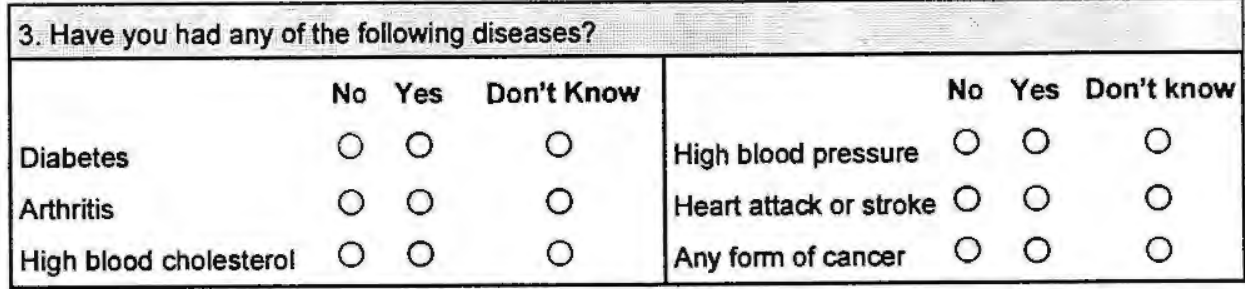

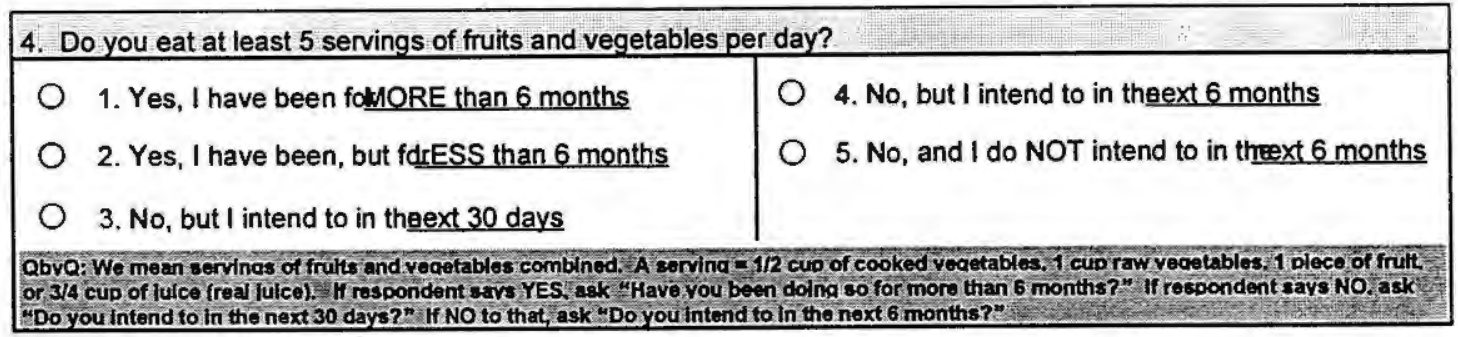


5. Do you consistently use a sunscreen with an SPF (sun orotection factor) of 15 or more whenever you know you will be out in the sun for more than 15 minutes?

1. Yes, I have been foltoRE than 12 months

2. Yes, I have been, but fdrESS than 12 months

3. No, but $I$ intend to in theext 30 days
4. No, but $I$ intend to in theext 12 months

5. No, and I do NOT intend to in thext 12 months

QbvQ: "Consistently means whenever vou know you wil be out in the sun for move than about 15 minutes, If respondent savs $Y E S$. ask "Have vou been doing so for more than 12 months?" If respondent savs NO. ask "DO vou intend to in the next 30 davs?" If NO to that ask

uDo you intend to in the next 12 menths?"

Please answer the following questions based on your experiences with smoking

6. Are you currently a cigarette smoker?

No $O$ Yes

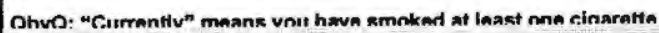
Skio Pattem: if YES (oo to Q9)

7. Were you ever a smoker?

No $O$ Yes Skip Pattem: If NO (go to Exercise: Stage form lQ13)

8. Have you smoked any cigarettes within the last 6 months?

No $O$ Yes Skip Pattem: If YES or NO (go to Exercise : Stage form (Q13)

9. Are you seriously considering quitting within the next six months?

No

Yes Skip Pattem: If NO, then go to 11.

10. Are planning to quit in the next 30 days?

No $O$ Yes

11. IN THE LAST YEAR how many times have you quit for at least 24 hours? (If more than 9 times, put 9 )

\begin{tabular}{ll|ll|l|l|ll|ll|ll|l|l|l|l|l|l|l|l|l|l|l|l}
0 & 0 & 0 & 1 & 0 & 2 & 0 & 3 & 0 & 4 & 0 & 5 & 0 & 6 & 0 & 7 & 0 & 8 & 0 & 9
\end{tabular} Qbva: If respondent provides a verv large number of quit attempts, verifv that thev rofer to the last vear and oach attempt lasted AT LEAST 24 hours.

12. Have you smoked ANY cigarettes, even a puff, in the PAST 24 HOURS?

No $O$ Yes

QbvQ: Anv part of a ciaarette and anv amount of smokina counts. 
The next series of questions are about your exercise habits. First, I need to define reqular exercise . Reqular exercise is any planned physical activity (e.q., brisk walking, aerobics, logaing, bicycling, swimming, rowing, etc.) performed to increase physical fitness. Such activity should be performed 3 to 5 times per week for $20-60$ minutes per session. Exercise does not have to be painful to be effective but should be done at a level that increases vour breathing rate and causes you to break a sweat

According to the definition of reqular exercise described. please answas or No for the following questions...

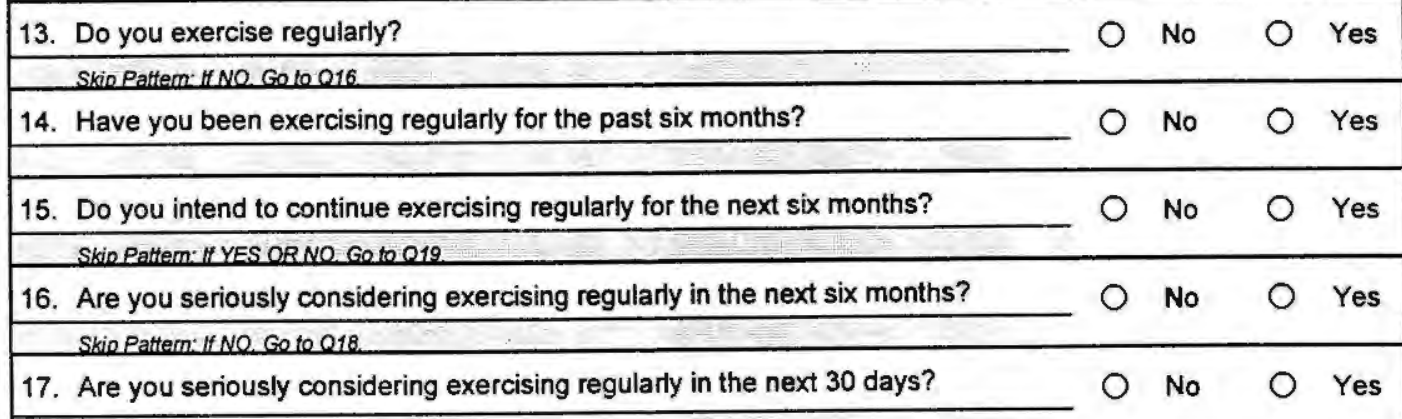

18. Although you said you do not exercise regularly, would you say you exercise some of No $\bigcirc$ Yes the time?

\section{LEISURE-TIME EXERCISE}

Considering a typical week ( 7 days) how many times on average did vou do the following kinds of exercisathan 20 minutes at a timeduring your free time (meaning that the exercise was not done during school or work)? (INTERVIEWERS: Please mark only one response for eamkestion)

19. STRENUOUS EXERCISE - HEART BEATS RAPIDLY, SWEATING (e.g., runnina. iogaing, hockey, soccer, squash, cross country sking. judo. roller skating, vigorous swimming, vigorous long distance bicycling. vigorous aerobic dance classes, heaw weight training) HOW MANY TIMES PER WEEK?

$\begin{array}{ccccccccccccccccccc}0 & 1 & 2 & 3 & 4 & 5 & 6 & 7 & 8 & 9 & 10 & 11 & 12 & 13 & 14 & 15 & 16 & 17+ \\ 0 & 0 & 0 & \bigcirc & \bigcirc & 0 & 0 & 0 & 0 & 0 & 0 & 0 & 0 & \bigcirc & \bigcirc & 0 & 0 & 0 & 0\end{array}$

20. MODERATE EXERCISENOT EXHAUSTING, LIGHT SWEATING (e.a.. fast walkina. baseball, tennis, easY bicvcling, vollevball, badminton, easv swimmina, alpine sking, popular and folk dancina) HOW MANY TIMES PER WEEK?

$\begin{array}{llllllllllllllllll}0 & 1 & 2 & 3 & 4 & 5 & 6 & 7 & 8 & 9 & 10 & 11 & 12 & 13 & 14 & 15 & 16 & 17+ \\ 0 & 0 & 0 & 0 & 0 & 0 & 0 & 0 & 0 & 0 & 0 & 0 & 0 & 0 & 0 & 0 & 0 & 0\end{array}$

21. MILD EXERCISE - MINIMAL EFFORT, NO SWEATING (e.q., easy walking, yoga, archery, fishing, bowiing, lawn bowling, shuffleboard, horseshoes, golf, snowmobiling) HOWMANY TIMES PER WEEK?

\begin{tabular}{ccccccccccccccccccc}
\hline 0 & 1 & 2 & 3 & 4 & 5 & 6 & 7 & 8 & 9 & 10 & 11 & 12 & 13 & 14 & 15 & 16 & $17+$ \\
0 & 0 & 0 & 0 & 0 & 0 & 0 & 0 & 0 & 0 & 0 & 0 & 0 & 0 & 0 & 0 & 0 & 0 &
\end{tabular}




\section{PHYSICALACTIVITYASSESSMENT}

On a usual weekday, Monday through Friday, how much time do you spend on the following activities? Please note that I am going to give vou five types of activity and the total time spent on the five activities should add up to 24 hours.

(INTERVIEWERS: Total hours on this page should add up to approximately 24 hours. Please choose only one response for each question.)

22. Vigorous activity for a usual weekday - Monday to Fridav . (e.g., digaing in the garden, strenuous sports. iogaing, aerobic dancing, sustained swimming, brisk walking, heaw carpentry, bicycling on hills, etc...) HOWMANY HOURS OUT OF 24 DO YOU SPEND ON THIS ACTIVITY ON A USUAL WEEKDAY?

$\begin{array}{ccccccccccccccccccc}0 & 1 & 2 & 3 & 4 & 5 & 6 & 7 & 8 & 9 & 10 & 11 & 12 & 13 & 14 & 15 & 16 & 17+ \\ 0 & 0 & 0 & 0 & 0 & 0 & 0 & 0 & 0 & 0 & 0 & 0 & 0 & 0 & 0 & 0 & 0 & 0 & 0\end{array}$

23. Moderate activity for a usual weekday - Mondav to Fridav . (e.g., housework, light sports, regular walking, golf, vard work, lawn mowing, painting, repairing, light carpentry, balliroom dancing, bicycling on level ground, etc...) HOW MANY HOURS OUT OF 24 DO YOU SPEND ON THIS ACTIVITY ON A USUAL WEEKDAY?

$\begin{array}{cccccccccccccccccccc}0 & 1 & 2 & 3 & 4 & 5 & 6 & 7 & 8 & 9 & 10 & 11 & 12 & 13 & 14 & 15 & 16 & 17+ \\ 0 & 0 & 0 & 0 & 0 & 0 & 0 & 0 & 0 & 0 & 0 & 0 & 0 & 0 & 0 & 0 & 0 & 0\end{array}$

24. Liaht activity for a usual weekday - Mondav to Friday . (e.g.. office work, driving a car, strolling, personal care, standing with little motion, etc...) HOW MANY HOURS OUT OF 24 DO YOU SPEND ON THIS ACTIVTTY ON A USUAL WEEKDAY?

\begin{tabular}{|cccccccccccccccccc|}
\hline WEEKDAY? & 1 & 2 & 3 & 4 & 5 & 6 & 7 & 8 & 9 & 10 & 11 & 12 & 13 & 14 & 15 & 16 & $17+$ \\
0 & 0 & 0 & 0 & 0 & 0 & 0 & 0 & 0 & 0 & 0 & 0 & 0 & 0 & 0 & 0 & 0 & 0 \\
\hline
\end{tabular}

25. Sittinq activity for a usual weekday - Monday to Friday . (e.g., eating, reading, desk work, watching TV, listening to radio, etc...) HOW MANY HOURS OUT OF 24 DO YOU SPEND ON THIS ACTIVITY ON A USUAL WEEKDAY?

$\begin{array}{lllllllllllllllllll}0 & 1 & 2 & 3 & 4 & 5 & 6 & 7 & 8 & 9 & 10 & 11 & 12 & 13 & 14 & 15 & 16 & 17+ \\ 0 & 0 & 0 & 0 & 0 & 0 & 0 & 0 & 0 & 0 & 0 & 0 & 0 & 0 & 0 & 0 & 0 & 0 & \end{array}$

26. Sleepina or Reclinina for a usual weekday - Monday to Friday. HOW MANY HOURS OUT OF 24 DO YOU SPEND ON THIS ACTIVITY ON A USUAL WEEKDAY?

\begin{tabular}{|cccccccccccccccccc|}
\hline 0 & 1 & 2 & 3 & 4 & 5 & 6 & 7 & 8 & 9 & 10 & 11 & 12 & 13 & 14 & 15 & 16 & $17+$ \\
0 & 0 & 0 & 0 & 0 & 0 & 0 & 0 & 0 & 0 & 0 & 0 & 0 & 0 & 0 & 0 & 0 & 0 \\
\hline
\end{tabular}




\section{PHYSICAL ACTIVITY ASSESSMENT (weekend)}

On a usual weekend DAY. Saturday or Sundav, how much time do vou spend on the following activities? Again, please note that I am going to give vou five types of activity and the total time spent on the five activities should add up to 24 hours.

(INTERVIEWERS: Total hours on this page should add up to aporoximately 24 hours. Please choose only one response for each question.)

27. Viaorous activity for a usual weekend-day. (e.g., digging in the garden, strenuous sports. iogging, aerobic dancing. sustained swimming. brisk walking, heavy carpentry, bicycling on hills, etc...) HOW MANY HOURS OUT OF 24 DO YOU SPEND ON THIS ACTIVITY ON A USUAL WEEKEND DAY?

\begin{tabular}{|cccccccccccccccccc|}
\hline 0 & 1 & 2 & 3 & 4 & 5 & 6 & 7 & 8 & 9 & 10 & 11 & 12 & 13 & 14 & 15 & 16 & $17+$ \\
0 & 0 & 0 & 0 & 0 & 0 & 0 & 0 & 0 & 0 & 0 & 0 & 0 & 0 & 0 & 0 & 0 & 0 \\
\hline
\end{tabular}

28. Moderate activity for a usual weekend-dav. (e.g., housework, light sports, regular walking, golf, yard work, lawn mowing, painting, repairing, light carpentry, ballroom dancing, bicycling on level ground, etc...) HOW MANY HOURS OUT OF 24 DO YOU SPEND ON THIS ACTIVITY ON A USUAL WEEKEND DAY?

\begin{tabular}{ccccccccccccccccccc}
\hline 0 & 1 & 2 & 3 & 4 & 5 & 6 & 7 & 8 & 9 & 10 & 11 & 12 & 13 & 14 & 15 & 16 & $17+$ \\
0 & 0 & 0 & 0 & 0 & 0 & 0 & 0 & 0 & 0 & 0 & 0 & 0 & $\bigcirc$ & 0 & 0 & 0 & 0
\end{tabular}

29. Liaht activity for a usual weekend-dav. (e.g.. office work, driving a car, strolling. personal care, standing with little motion, etc...) HOW MANY HOURS OUT OF 24 DO YOU SPEND ON THIS ACTIVITY ON A USUAL WEEKEND DAY?

\begin{tabular}{|cccccccccccccccccc|}
\hline 0 & 1 & 2 & 3 & 4 & 5 & 6 & 7 & 8 & 9 & 10 & 11 & 12 & 13 & 14 & 15 & 16 & $17+$ \\
0 & 0 & 0 & 0 & 0 & 0 & 0 & 0 & 0 & 0 & 0 & 0 & 0 & 0 & 0 & 0 & 0 & 0 \\
\hline
\end{tabular}

\begin{tabular}{|c|c|c|c|c|c|c|c|c|c|c|c|c|c|c|c|c|c|}
\hline \multicolumn{18}{|c|}{$\begin{array}{l}\text { 30. Sittina activity for a usual weekend-dav. (e.g., eating, reading, desk work, watching TV, listening to radio, etc...) } \\
\text { HOW MANY HOURS OUT OF } 24 \text { DO YOU SPEND ON THIS ACTIVITY ON A USUAL WEEKEND DAY? }\end{array}$} \\
\hline 0 & 1 & 2 & 3 & 4 & 5 & 6 & 7 & 8 & 9 & 10 & 11 & 12 & 13 & 14 & 15 & 16 & $17+$ \\
\hline 0 & 0 & 0 & 0 & 0 & 0 & 0 & 0 & 0 & 0 & 0 & 0 & 0 & 0 & 0 & 0 & 0 & 0 \\
\hline
\end{tabular}

31. Sleevina or Reclining for a usual weekend-dav. HOW MANY HOURS OUT OF 24 DO YOU SPEND ON THIS ACTIVITY ON A USUAL WEEKEND DAY?

\begin{tabular}{|cccccccccccccccccc|}
\hline 0 & 1 & 2 & 3 & 4 & 5 & 6 & 7 & 8 & 9 & 10 & 11 & 12 & 13 & 14 & 15 & 16 & $17+$ \\
0 & 0 & 0 & 0 & 0 & 0 & 0 & 0 & 0 & 0 & 0 & 0 & 0 & 0 & 0 & 0 & 0 & 0 \\
\hline
\end{tabular}




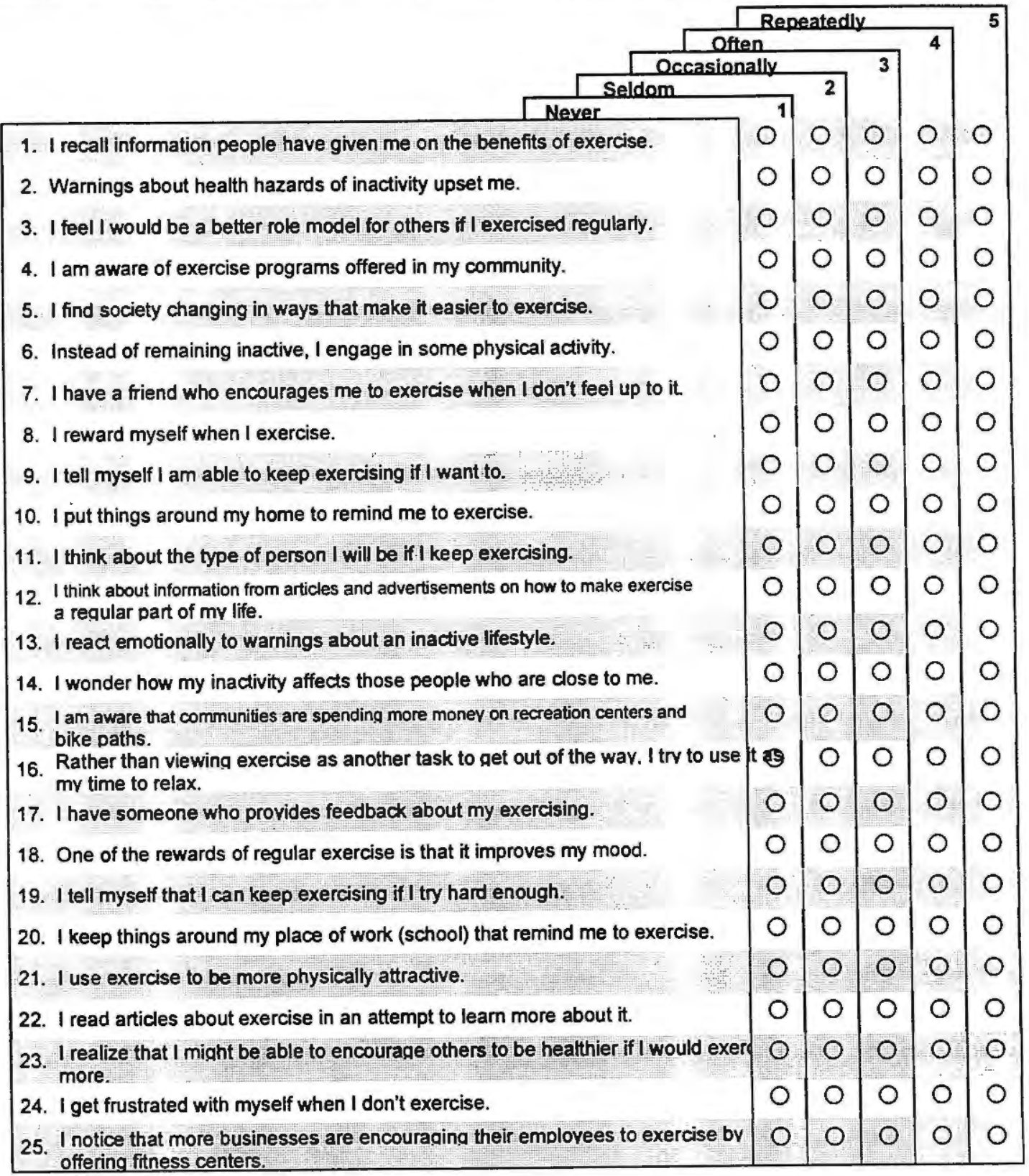




26. When I feel tired. I make myself exercise anwwav because I know I will feel better
afterwards.
27. I have a workout partner.
28. I get upset when I see people who would benefit from exercise but choose not
to exercise.
29. I make commitments to exercise.
30. I avoid spending long periods of time in environments that promote inactivity.
31. I have noticed that many people know that exercise is good for them.
32. I feel that I have to exercise to look my best.



experiences you mav be currently having or have had DURING THE PAST MONTH. Then rate how frequently the event occurs, according to the following 5 point scale:

51. I have joined a health club or fitness center to exercise regularly.
52. I bring my athletic shoes to work so I will remember to take a walk at lunch time. 0
53. I fear what would happen to my physical appearance if I stopped exercising.
54. I use my calendar to schedule my exercise time.


This next section looks at positive and negative aspects of exercise. Please indicate how important each statement is with respect to your decision to exercise or not to exercise in your leisure time according to the following 5 -point scale:

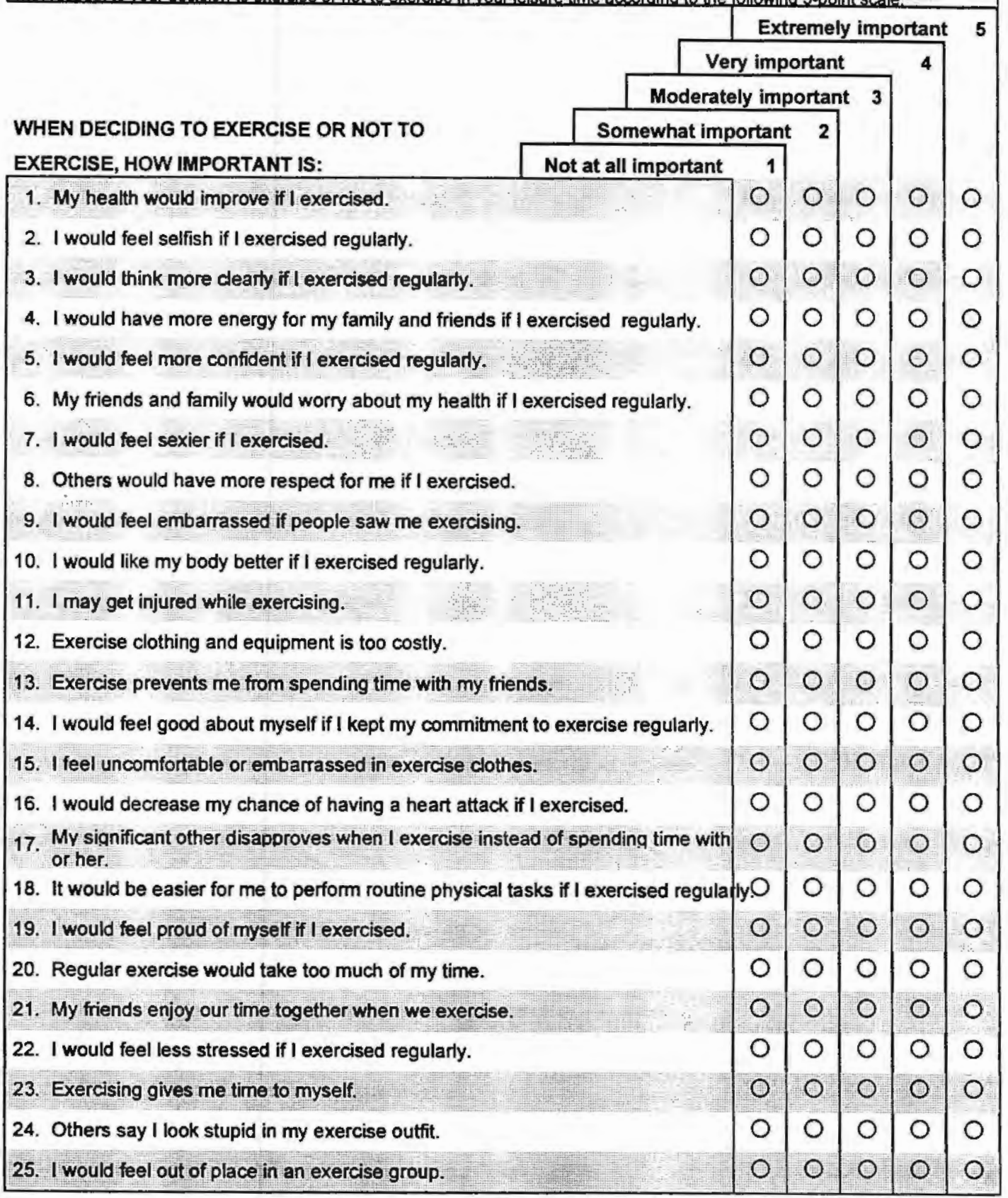


This next section looks at positive and negative aspects of exercise. Please indicate how important each statement is with respect to vour decision to exercise or not to exercise in vour leisure time according to the following 5-point scale

WHEN DECIDING TO EXERCISE OR NOT TO EXERCISE, HOW IMPORTANT IS:

\section{Exercise would help me lose weight.}

27. I would have less time for some of my favorite activities.

28. Exercising puts me in a better mood for the rest of the day.

29. Exercise prevents me from spending quality time with my family.

30. I would feel more comfortable with my body.

31. I am too out of shape to exercise.

32. Exercise puts an extra burden on my significant other.

33. My friends do not like when I exercise.

34. Exercise would lower my anxiety.

35. I think I would be too tired to do my daily work after exercising.

36. I would have more self respect if I exercised.

37. Mv friends and/or family would sav I spend too much money on exercise related $O$ things.

38. Regular exercise would help me have a more positive outlook on life.

39. There is too much I would have to learn to exercise.

40. Others would see me as sexier if I exercised.

41. I would look better if 1 exercised.

42. I feel uncomfortable when I exercise because $I$ get out of breath and $m v$ heart verv fast.

43. I feel better about myself.

44. Exercise allows me to participate in activities with my friends.

45. I am afraid to find that I am not good at exercising.

46. After exercising, my family and friends have to wait for me to shower.

47. Exercise is too boring to do it regularty.

48. I feel a sense of accomplishment after l exercise.

49. Others make fun of me when I exercise.
Extremely important 5

Very important

Moderately important 3

Somewhat important 2

Not at all important

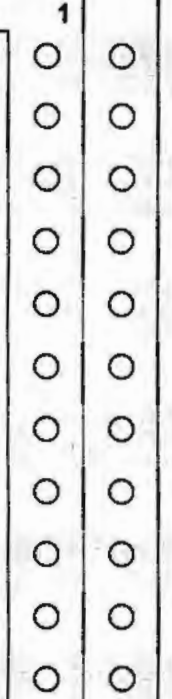

\begin{tabular}{l|l|l|l|l}
0 & 0 & 0
\end{tabular}

000

0000

0 o 0

0 - 00

0 - 00

0 - 00

० 00

0 O

- 00

0 o 0

000

$\begin{array}{ll}0 & 0 \\ 0 & 0\end{array}$

O 

Next are some situations in which some people might choose not to exercise when other things get in the way. Please
rate how confident you are that you would participate in regular exercise in your leisure time, according to the following 5 point scale:

Completely confident 5

I AM CONFIDENT THAT I CAN PARTICIPATE IN REGULAR EXERCISE WHEN...

1. I am tired.

2. I am in a bad mood.

3. I feel I don't have the time.

4. I am on vacation.

5. The weather is bad.

6. I am under a lot of stress.

7. I am feeling lazy.

8. My friends call me to go out.

9. There is a good program on TV.

10. I am alone.

11. I have to commute to exercise.

12. I don't have access to exercise equipment.

13. I am busy.

14. My friends don't want me to exercise.

15. I am depressed.

16. I am anxious.

17. I am happy.

18. My significant other does not want me to exercise.

19. I am spending time with friends or family who do not exercise.

20. I am travelling.

21. My gym is closed.

22. I've had a bad day.

23. I have to exercise alone.

24. My exercise partner decides not to exercise that day.

25. I don't feel like it.

Very confident

Moderately confident 3

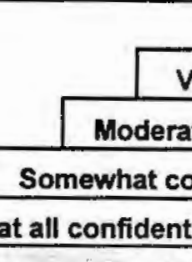

Not at all confident

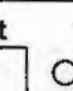

$0^{1} 0$

$0\left|\begin{array}{l|l}0 \\ 0\end{array}\right|$

0100

0100

000

$0|0| 0$

000

$010 / 0$

000

0100

00

0

00

0 o

00

00

00

0

00

0

- 0

- 0

00

0

00 


\section{EXERCISE HISTORY}

The next series of questions ask about your past exercise history. Whether or not you are physically active now, it is
possible that you have exercised regularly in the past. Please answer these questions only considering your PAST
exercise history. NOT your current exercise program.

2. In the past 5 years how many supervised exercise programs have you participated in that lasted MORE than 6 months?

A PERSONAL exercise program is regular exercise which you do on your own or with others, but is not supervised or formally organized (e.g. jogging, biking, swimming).

3. In the past 5 years how many personal exercise programs have you participated in that lasted LESS than 6 months?

4. In the past 5 years how many personal exercise programs have you participated in that lasted MORE than 6 months?

5. Out of the past 5 years, approximately what is the TOTAL in years that you have exercised regularly?

$Q$ by $Q$ : Read all options. Remind respondent of the definition of regular exercise if he/she is not sure how to respond. Regular exercise is any planned physical activity (e.g., brisk walking, aerobics, jogging, bicycling, swimming, rowing, etc.) performed to increase physical fitness. Such activity should be performed 3 to 5 times per week for 20-60 minutes per session. Exercise does not have to be painful to be effective but should be done at a level that increases your breathing rate and causes you to break a sweat.

Less than 1 year.

1 to 2 years.

2 to 3 years.
3 to 4 years.

O 4 to 5 years. 


\section{Demoaraphics}

Instructions:Please answer the following questions based on your present situation.

\begin{tabular}{|cccc|}
\hline $\begin{array}{r}\text { 1. What is vour age? } \\
\text { (Please enter as a 2-diait } \\
\text { number, i.e. 00. 01, } 02 \text {, etc.) }\end{array}$ \\
\hline \multicolumn{3}{|c|}{ YEARS } \\
\hline 0 & 0 & 0 & 0 \\
1 & 0 & 1 & 0 \\
2 & 0 & 2 & 0 \\
3 & 0 & 3 & 0 \\
4 & 0 & 4 & 0 \\
5 & 0 & 5 & 0 \\
6 & 0 & 6 & 0 \\
7 & 0 & 7 & 0 \\
8 & 0 & 8 & 0 \\
9 & 0 & 9 & 0 \\
\hline
\end{tabular}

\begin{tabular}{|ccccccc|}
\hline 2. $\begin{array}{l}\text { How much do vou weiah? } \\
\text { (Please enter as a 3-diait } \\
\text { number, i.e. } 097,168 \text {, etc. }\end{array}$ \\
\hline \multicolumn{5}{c}{ POUNDS } \\
\hline 1 & 0 & 0 & 0 & 0 & 0 \\
2 & 0 & 2 & 0 & 2 & 0 \\
3 & 0 & 3 & 0 & 3 & 0 \\
4 & 0 & 4 & 0 & 4 & 0 \\
5 & 0 & 5 & 0 & 5 & 0 \\
6 & 0 & 6 & 0 & 6 & 0 \\
7 & 0 & 7 & 0 & 7 & 0 \\
8 & 0 & 8 & 0 & 8 & 0 \\
9 & 0 & 9 & 0 & 9 & 0 \\
\hline
\end{tabular}

\begin{tabular}{|c|c|c|c|c|c|}
\hline 3. & $\begin{array}{l}\text { Hor } \\
\text { (Ple } \\
\text { inch }\end{array}$ & $\begin{array}{l}\text { tall a } \\
\text { se e } \\
\text { s.) }\end{array}$ & $\begin{array}{l}\text { you } \\
\text { er fe }\end{array}$ & t and & \\
\hline FEE & & INC & IES & $-\quad$ & \\
\hline 0 & 0 & 0 & 0 & 10 & \\
\hline 1 & 0 & 1 & 0 & 11 & 0 \\
\hline 2 & 0 & 2 & 0 & & \\
\hline 3 & 0 & 3 & 0 & & \\
\hline 4 & 0 & 4 & 0 & & \\
\hline 5 & 0 & 5 & 0 & & \\
\hline 6 & 0 & 6 & 0 & & \\
\hline 7 & 0 & 7 & 0 & & \\
\hline 8 & 0 & 8 & 0 & & \\
\hline 9 & 0 & 9 & 0 & & \\
\hline
\end{tabular}

\begin{tabular}{|c|c|c|c|c|c|c|c|c|c|c|c|c|c|}
\hline Grade School & 1 & 0 & 2 & 0 & 3 & 0 & 4 & 0 & 5 & 0 & 60 & 7 & 0 \\
\hline High School & 8 & 0 & 9 & 0 & 10 & 0 & 11 & 0 & 12 & 0 & & & \\
\hline College & 1 & 0 & 2 & 0 & 3 & 0 & 4 & 0 & 5 & 0 & & & \\
\hline Graduate School & 1 & 0 & 2 & 0 & 3 & 0 & 4 & 0 & $5+$ & 0 & & & \\
\hline
\end{tabular}

5. What is your manital status? (please read all choices)

\begin{tabular}{ll|ll|rr}
0 & 1. Married & 0 & 3. Divorced & 0 & 5. Separated \\
0 & 2. Not married & 0 & 4. Not married, but living with a partngr & 0 & 6. Widowed
\end{tabular}




\section{Demographics Continued}

\begin{tabular}{|c|c|c|c|c|c|}
\hline \multicolumn{6}{|c|}{ 6. What is your race? (please read all choices) } \\
\hline $\mathrm{O}$ & 1. White & $\mathrm{O}$ & 3. American Indian, Alaskan Native & $\mathrm{O}$ & 5. Other \\
\hline 0 & 2. Black & 0 & 4. Asian, Pacific Istander & & \\
\hline
\end{tabular}

\begin{tabular}{|c|c|c|c|c|}
\hline 7. Are you of Spanish/Hispanic origin? & $\mathrm{O}$ & 1. No & $\mathrm{O}$ & 2. Yes \\
\hline
\end{tabular}

8. Which of the following best describes your employment status? (please read all choices)

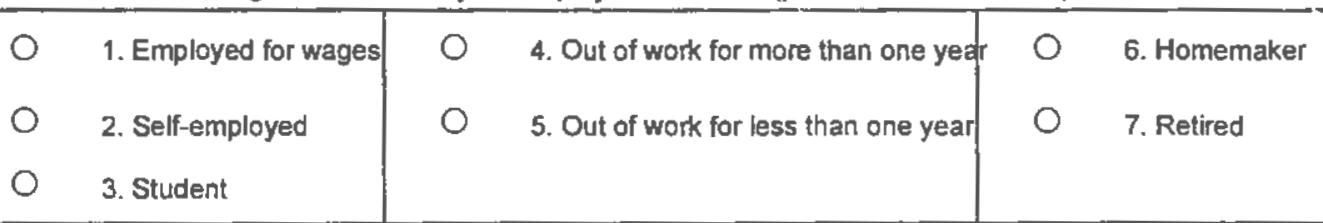

9. Which of the following categories best describes your annual household income from all sources? (please read all choices)

\begin{tabular}{ll|ll}
\hline Under 15,000 & $O$ & $40,000-59,999$ \\
$O$ & $15,000-29,999$ & $O$ & $60,000-79,999$ \\
$O$ & $30,000-39,999$ & $O$ & 80,000 and over
\end{tabular}




\section{Bibiliography}

Aldenderfer, M. S. \& Blashfield, R. K. (1984). Cluster Analysis. Sage Publications, Beverly Hills: CA.

Allport, G. (1937). Personality: A psychological interpretation. New York: Holt.

American College of Sports Medicine. (1990). Position stand on the recommended quantity and quality of exercise for developing and maintaining cardiorespiratory and muscular fitness healthy adults. Medicine and Science in Sports and Exercise, 22, 265-274.

American Heart Association. (1997, December). URL http://www.amhrt.org/heart and_stroke_A_Z_guide/cvds.html

Arthitis Foundation. (1997, December). URL http://www.arthritis.org/facts

Bandura, A. (1977). Toward a unifying theory of behavior change. Psychological Review, 84, 191-215.

Bandura, A. (1982). Self-Efficacy mechanism in human agency. American Psychologist, 37, 122-147.

Benisovich, S. V., Rossi, J. S., Norman, G. J., \& Nigg, C. R. (1998). A multidimensional approach to exercise self-efficacy: Relationship with exercise behavior and attitudes toward exercise. Paper presented at the meeting of the Eastern Psychological Association, Boston, MA. 
Blair, S. N. (1993). 1993 C.H. McCloy Research Lecture: Physical activity, physical fitness and health. Research Quarterly for Exercise and Sport, 64, 365-376.

Blashfield, R. K. (1976). Mixture model tests of cluster analysis: Accuracy of four agglomerative hierarchical methods. Psychological Bulletin, $\underline{83}, 377-388$.

Booth, M. L., Macaskill, P., Owen, N., Oldenburg, B., Marcus, B., \& Bauman, A. (1993). Population prevalence and correlates of stages of change in physical activity. Health Education Quarterly, 20, 431-440.

Boyle, R. G., O’Conner, P. J., Pronk, N. P., \& Tan, A. (1998). Stages of change for phyical activity, diet, and smoking among HMO members with chronic conditions. American Journal of Public Health, 12, 170-175.

Breckenridge, J. N. (1989). Replicating cluster analysis: Method, consistency, and validity. Multivariate Behavorial Research, 24, 147-161,

Brownell, K. D., Marlatt, C. A., Lichtenstein, E., \& Wilson, C. T. (1986). Understanding and preventing relapse. American Psychologist, 41, 756-782.

Buxton, K. E., Mercer, B. H., Wyse, B., \& Ashford, B. (1994). Evidence for the concurrent validity and measurement reliability of a stages of exercise behavior change instrument. Journal of Sport and Exercise Psychology, 16, S35.

Buxton, K., Wyse, J., \& Mercer, T. (1996). How applicable is the stages of change model to exercise behaviour? A review. Health Education Journal, 55, 239257.

Calinski, R. B., \& Harabasz, J. (1974). A dendrite method for cluster analysis. Communications in Statistics, 3, 1-27. 
Cardinal, B. J. (1995). The stages of exercise scale and stages of exercise behavior in female adults. The Journal of Sports Medicine and Physical Fitness, 35, $87-92$.

Cardinal, B. J. (1997). Construct validity of stages of change for exercise behavior. American Journal of Health Promotion, 12, 68-74.

Centers for Disease Control and Prevention. (1997, August). CDC Surveillance Summaries. Morbidity and Mortality Weekly Report 1997, 46 (No. SS-3).

Cohen, J. (1960). A coefficient of agreement for nominal scales. Educational and Psychological Measurement, 20, 37-46.

Cohen, J. (1992). A power primer. Psychological Bulletin, 112, 155-159. Cronbach, L. J. (1957). The two disciplines of scientific psychology. American Psychologist, 12, 671-684.

Cronbach, L. J., \& Gleser, G. C. (1953). Assessing the similarity between profiles. Psychological Bulletin, 50, 456-473.

DiClemente, C. C, Prochaska, J. O., Fairhurst, S. K., Velicer, W. F., Velasquez, M M., Rossi, J. S. (1991). Journal of Consulting and Clinical Psychology, 59, 295-304. Dishman, R. K. (1988). Exercise Adherence: its Impact on Public Health. Champaign, IL:Human Kinetics.

Dishman, R. K. (1990). Determinants of participation in physical activity. In C. Bouchard, R.J. Shephard, T. Stephens, J.R. Sutton, B.D. McPherson (Eds.), Exercise, fitness and health. Champaign, IL: Human Kinetics Publishers. 
Dishman, R. K. (1994). The measurement conundrum in exercise adherence research. Medicine and Science In Sports and Exercise, 26, 1382-1390.

Dishman, R. K., Sallis, J. F. (1993). Determinants and interventions for physical activity and exercise. In C. Bouchard, R.J. Shephard, T. Stephens (Eds.), Physical activity, fittness, and Health: Consensus statment. Champaign, IL: Human Kinetics Publishers.

Dunn, J. G. (1994). Toward the combined use of nomothetic and idiographic methodologies in sport psychology: An empirical example. Sport Psychologist, $\underline{8}, 376-$ 392.

Dzewaltowski, D. A. (1994). Physical activity determinants: a social cognitive approach. Medicine and Science In Sports and Exercise, 26, 1395-1399.

Edelbrock C. (1979). Mixture model tests of hierarchical clustering algorithms: the problem of classifying everybody. Multivariate Behavioral Research, 14, 367-384.

Everitt, G. (1981). Cluster Analysis. New York: Halsted Press.

Fava, J. L., Velicer, W. F., Prochaska, J. O., (1995). Applying the transtheoretical model to a representative sample of smokers. Addictive Behaviors, 20, 189-203.

Franck, I. (1982). Psychology as a science: Resolving the idiographicnomothetic controversy. Journal for the Theory of Social Behaviour, 12, 1-20.

Godin, G. \& Shepard, R. J. (1985). A simple method to assess exercise behavior in the community. Canadian Journal of Applied Sport Sciences, 10, 141-146. 
Godin, G. \& Shepard, R. J. (1990). Use of attitude-behaviour models in exercise promotion. Sports Medicine, 10, 103-121.

Godin, G. (1994). Theories of reasoned action and planned behavior: usefulness for exercise promotion. Medicine and Science in Sports and Exercise, 26, 1391-1394.

Godin, G., Jobin, J. \& Bouillon, J. (1986). Assessment of leisure time exercise behavior by self-report: A concurrent validity study. Canadian Journal of Public Health, 77, 359-362.

Gordon, A. D. (1981). Classification: Methods for the Exploratory Analysis of Multivariate Data. London: Chapman and Hall.

Gorely, T., \& Gordon, S. (1995). An examination of the transtheoretical model and exercise behavior in older adults. Journal of Sport and Exercise Psychology, 17, 312324.

Heather, N. (1991). Foreword. In R. Davidson, S. Rollnick, \& I. MacEwan (Eds.), Counseling problem drinkers (p. x - xi). New York: Routledge.

Hesser, J. E. \& Kim, H. (1997). Levels of phyical activity among Rhode Island adults. Medicine and Health / Rhode Island, 으, 280-285.

Howard, G. S. \& Myers, P. R. (1990). Predicting human behavior: Comparing idiographic, nomothetic, and agentic methodologies. Journal of Counseling Psychology, 37, 227-233.

Humphreys, K. \& Rosenheck, R. (1995). Sequential validation of cluster analytic subtypes of homeless veterans. American Journal of Community Psychology, 23, 75- 97. 
Janis, I. L., \& Mann, L. (1968). A conflict theory approach to attitude and decision making. In A. Green wald, T. Brook, \& T. Ostrom (Eds.), Psychological Foundations and Attitudes. NY: Academic Press.

King, A. C., Blair, S. N., Bild, D. E., Dishman, R. K., Dubbert, P. M., Marcus, B. H., et al. (1992). Determinants of physical activity and interventions in adults. Medicince and Science in Sports and Exercise, 24, S221-S236.

Kluckhohn, C., Murray, H. A., \& Schneider, D. M. (Eds.). (1953).

Personality in nature, society, \& culture (2nd ed.). New York: Knopf.

Laforge, R. G. (1992). [Health risk factor screening of a New England managed care organization]. Unpublished raw data.

Laforge, R. G. (1998). [Baseline multiple risk behavior intervention data from New England worksites]. Unpublished raw data.

Laforge, R. G., Greene, G. W., \& Prochaska, J. O. (1994). Psychosocial factors influencing low fruit and vegetable consumption. Journal of Behavioral Medicine, 17, 361-374.

Lautenschlager, G. J. (1989). A comparison of alternatives to conducting Monte Carlo analyses for determining parallel analysis criteria. Multivariate Behavioral Research, 24, 365-395.

Lorr, M. (1983). Cluster Analysis For Social Scientists. San Francisco: Jossey-Bass Publishers.

Marcus, B. H., \& Owen, N. (1992). Motivational readiness, self-efficacy, and decision-making for exercise. Journal of Applied Social Psychology, 22, 3-16. 
Marcus, B. H., Banspach, S. W., Lefebvre, R. C., Rossi, J. S., Carleton, R. A., \& Abrams, D. B. (1992). Using the stages of change model to increase the adoption of physical activity among community participants. American Journal of Health Promotion, 6, 424-429.

Marcus, B. H., Pinto, B. M., Simkin, L. R., Audrain, J. E., \& Taylor, E. R. (1994). Application of theoretical models to exercise behavior among employed women. American Journal of Health Promotion, 2, 49-55.

Marcus, B. H., Rakowski, W., \& Rossi, J. S. (1992). Assessing motivational readiness and decision making for exercise. Health Psychology, 11, 257-261.

Marcus, B. H., Rossi, J. S., Selby, V. C., Niaura, R. S., Abrams, D. B. (1992). The stages and processes of exercise adoption and maintenance in a worksite sample. Health Psychology, 11, 386-395.

Marcus, B. H., Selby, V. C., Niaura, R. S. Rossi, J. S. (1992). Self efficacy and the stages of exercise behavior change. Research Quarterly for Exercise and Sport, 63 , 60-66.

Marcus, B. H., Simkin, L. R. (1993). The stages of exercise behavior. The Journal of Sports Medicine and Physical Fitness, 33, 83-88.

Marcus, B. H., Simkin, L. R. (1994). The transtheoretical model: applications to exercise behavior. Medicine and Science in Sports and Exercise, 26, 1400-1404.

McArdle, J. J., \& Epstein, D. B. (1987). Latent growth curves within developmental structural equation models. Child Development, 58, 110-133. 
McIntyre, R. M. \& Blashfield, R. K. (1980). A nearset-centroid technique for evaluating the minimum-variance clustering procedure. Multivariate Behavioral Research, 2, 225-238.

Milligan, G. W. \& Cooper, M. C. (1985). An examination of procedures determining the number of clusters in a data set. Psychometrika, 50, 159-179.

Milligan, G. W. (1980). An examination of the effect of six types of error perturbation of fifteen clustering algorithms. Psychometrika, 45, 325-342.

Milligan, G. W. (1981a). A review of Monte Carlo tests of cluster analysis. Multivariate Behavioral Research , 16, 379-407.

Milligan, G. W. (1981b). A Monte Carlo study of thirty internal criterion measures for cluster analysis. Psychometrika, 46, 187-199.

Milligan, G. W. \& Cooper, M. C. (1987). Methodology review: Clustering methods. Applied Psychological Measurement, 11, 329-354.

Montoye, H. J., Kemper, H. S. G., Saris, W. H. M., \& Washburn, R. A. (1996). Measuring physical activity and energy expenditure. Champaign, IL:Human Kinetics.

Morey, L. C., Blashfield, R. K., Skinner, H. A. (1983). A comparision of cluster analysis techniques within a sequential validation framework. Multivariate Behavioral Research, 18, 309-329.

Nigg, C. R., \& Courneya, K. S. (1997). Transtheoretical model: Examining adolescent exercise behavior. Jouranl of Adolescent Health, 
Norman, G. J., Velicer, W. F., Fava, J. L., \& Prochaska, J. O. (1997). Cluster subtypes within stage of change in a representative sample of smokers. Manuscript under review.

Overall, J. E. \& Magee, K. N. (1992). Replication as a rule for determining the number of clusters in hierarchial cluster analysis. Applied Psychological Measurement, 16, 119-128.

Paffenbarger, R. S., Blair, S. N., Lee, I., \& Hyde, R. T. (1993). Measurement of phyical activity to assess health effects in free-living populations. Medicine and Science in Sport and Exercise, 25, 60-70.

Pallonen, U. E., Velicer, W. F., Prochaska, J. O., Bellis, J. M., Tsoh, J. Migneault, J. P., Smith, N. F., Prokhorov, A. V. (1998). Computer-based smoking cessation interventions in adoliscents: Description, feasibility, and six-month follow-up findings. Substance Use \&. Misuse, 33, 1-31.

Pate, R. R., Pratt, M., Blair, S. N., Haskell, W. L., Macera, C. A., Bouchard, C., et al. 1995. Physical activity and public health: A recommendation from the centers for disease control and prevention and the american college of sports medicine. Journal of the American Medical Association, 273, 402-407.

Phillips, D. C. (1987). Philosophy, science, and social inquiry. Oxford: Pergamon Press.

Prochaska, J. O. (1979). Systems of pychotherapy: A transtheoretical analysis. Homewood, IL: Dorsey Press. 
Prochaska, J. O. (1994). Strong and weak principles for progressing from precontemplation to action on the basis of twelve problem behaviors. Health Psychology, $13,47-51$.

Prochaska, J. O. (1996). A stage paradigm for integrating clinical and public health approaches to smoking cessation. Addictive Behaviors, $21,721-732$.

Prochaska, J. O. \& Marcus, B. H. (1995). The transtheoretical model: Applications to exercise. In R.K. Dishman (ed), Advances in Exercise Adherence. Chgampaign, IL: Human Kinetics.

Prochaska, J. O., DiClemente, C. C. (1983). Stages and processes of self change in smoking: Towards an integrative model of change. Joumal of Consulting and Clinical Psychology, 51, 390-395.

Prochaska, J. O., DiClemente, C. C., Norcross, J. C. (1992). In search of how people change: Applications to addictive behaviors. American Psychologist, 47, $1102-$ 1114.

Prochaska, J. O., Velicer, W. F., DiClemente, C. C., \& Fava, J. L. (1988). Measuring processes of change: Applications to the cessation of smoking. Journal of Consulting and Clinical Psychology, 56, 520-528.

Prochaska, J. O., Velicer, W. F., Guadagnoli, E., Rossi, J. S., DiClemente, C. C. (1991). Patterns of change: Dynamic typology applied to smoking cessation. Multivariate Behavioral Research, 26, 83-107.

Prochaska, J. O., Velicer, W. F., Rossi, J. S., Goldstein, M. G., Marcus, B. H., Rakowski, W., Fiore, C., Harlow, L. L, Redding, C. A., Rosenbloom, D., \& Rossi, S. R. 
(1994). Stages of change and decisional balance for 12 problem behaviors. Health Psychology, 13, 39-46.

Rapkin, B. D. (1993). Cluster analysis in community research: Epistemology and practice. American Journal of Community Psychology, 21, 247-277.

Raykov, T. (1997). Simultaneous study of individual and group patterns of latent longitudinal change using structural equation modeling. Structural Equation Modeling, 4, 212-236.

Reed, G. R. (1995). Measuring stage of change for exercise. Unpublished doctoral dissertation..

Reed, G. R., Velicer, W. F., Prochaska, J. O., Rossi, J. S., \& Marcus, B. H. (1997). What makes a good staging algorithms: examples from regular exercise. American Journal of Health Promotion, 12, 57-66.

Rossi, J. S., Blais, L. M., Redding, C. A., \& Weinstock, M. A. (1995). Preventing skin cancer through behavior change: Implications for interventions. Dermatologic Clinics, 13, 613-622.

Rossi, J. S., Norman, G. J., Nigg, C. R., \& Benisovich, S. V. (1998). Refinement and further development of transtheoretical model measures of exercise behavior change. Manuscript in preparation.

Rossi, J. S., Weinstock, M. A., Redding, C. A., \& Cottrill, S. D. (1996). Effectiveness of stage-matched interventions for skin cancer prevention. Paper presented at the American Public Health Association Meeting. 
Ruggiero, L., Redding, C. A., Rossi, J. S., Prochaska, J. O. (1997). A stagematched smoking cessation program for pregnant smokers. American Journal of Health Promotion, 12, 31-33.

Sallis, J. F., \& Hovell, M. F. (1990). Determinants of exercise behavior, Exercise and Sport Science Review, 18, 307-330.

Sallis, J. F., Pinski, R. B., Patterson, T. L., \& Nader, P. R. (1988). The development of self-efficacy scales for health-related diet and exercise behaviors. Health Education Research , 3, 283-292.

Sarle, W. S. (1983). Cubic clustering criterion. SAS Technical Report A108.

SAS Institute Inc. (1988). SAS/STAT User's Guide: Release 6.03 Edition. Cary, NC: SAS Institute Inc.

Skinner, C. S., Strecher, V. J., Hospers, H. (1994). Physicians' recommendations for mamography: Do tailored messages make a difference? American Journal of Public Health, 84, 43-49.

Sonstroem, R. J. (1988). Psychological models. In R. K. Dishman (ed), Exercise Adherence: It Impact on Public Health, Champaign, IL: Human Kinetics, 123-154.

Strecher, V. J., Kreuter, M. K., Den Boer, K. J., Kobrin, S., Hospers, H. J., Skinner, C. S. (1994). The effects of comuter-tailored cessation meessages in family practice settings. The Journal of Eamily Practice, 39, 262-270. 
Velicer, W. F. (1976). Deternining the number of components from the marix of partial correlations. Psychometrika, 41, 321-327.

Velicer, W. F., DiClemente, C. C, Rossi, J. S., \& Prochaska, J. O. (1990). Relapse situations and self-efficacy: An integrative model. Addictive Behaviors, 15, 271283.

Velicer, W. F., DiClemente, C. C., Prochaska, J. O., Brandenburg, N. (1992). Decisional balance measure for assessing and predicting smoking status. Journal of Personality and Social Psychology, 48, 1279-1289.

Velicer, W. F., Fava, J. L., Prochaska, J. O., Abrams, D. B., Emmons, K. M., \& Pierce, J. P. (1995). Distribution of smokers by stage in three representative samples. Preventive Medicine, 24, 401-411.

Velicer, W. F., Hughes, S. L., Fava, J. L., Prochaska, J. O., \& DiClemente, C. C. (1995). An empirical typology of subjects within stage of change. Addictive Behaviors, $\underline{20}, 299-320$.

Velicer, W. F., Prochaska, J. O., Bellis, J. M., DiClemente, C.C., Rossi, J. S., Fava, J. L., \& Steiger, J. H. (1993). An expert system intervention for smoking cessation. Addictive Behaviors, 18, 269-290.

Velicer, W. F., Rossi, J. S., Prochaska, J. O., \& DiClemente, C. C. (1996). A criterion measurement model for health behavior change. Addictive Behaviors, 21, 555584.

Velicer, W. F., Rossi, J. S., Ruggiero, L., \& Prochaska, J. O. (1994). Minimal interventions appropriate for an entire population of smokers. In R. Richmond (Ed.), 
Interventions for Smokers: An International Perpective. Baltimore: Williams \& Wilkins, 69-92.

Waller, N. G. \& Meehl, P. E. (1998). Multivariate taxometric procedures: Distinguishing types from continua. Thousand Oaks: Sage Publications.

Ward, J. H. (1963). Hierarchical grouping to optimize an objective function. Joumal of the American Statistical Association, 58, 236-244.

Washburn, R. A., Smith, J. W., Goldfield, S. R, \& McKinley, J. B. (1991). Reliability and physiologic correlates of the Harvard Alumni Activity Survey in a general population. Journal of Clinical Epidemiology, 44, 1319-1326. 